Ann. Scient. Éc. Norm. Sup.,

$4^{e}$ série, t. 40, 2007, p. 901 à 949.

\title{
HYPERBOLIC COMPONENTS OF POLYNOMIALS WITH A FIXED CRITICAL POINT OF MAXIMAL ORDER
}

\author{
By PASCALE ROESCH ${ }^{1}$
}

ABSTRACT. - For the study of the 2-dimensional space of cubic polynomials, J. Milnor considers the complex 1-dimensional slice $\mathcal{S}_{n}$ of the cubic polynomials which have a super-attracting orbit of period $n$. He gives in [15] a detailed and partially conjectural picture of $\mathcal{S}_{n}$. In the present article, we prove these conjectures for $\mathcal{S}_{1}$ and generalize these results in higher degrees. In particular, this gives a description of the closures of the hyperbolic components and of the Mandelbrot copies sitting in the connectedness locus. We prove that the boundary of each hyperbolic component is a Jordan curve, the points of which are characterized according to the dynamical behaviour of the associated polynomial. The global picture of the connectedness locus is a closed disk together with "limbs" sprouting off it at the cusps of Mandelbrot copies and whose diameter tends to 0 (which corresponds to a qualitative Yoccoz' inequality).

(C) 2007 Elsevier Masson SAS

RÉSUMÉ. - Pour étudier l'espace 2-dimensionnel des polynômes cubiques, J. Milnor considère la tranche $\mathcal{S}_{n}$ de dimension un complexe formée des polynômes cubiques qui ont une orbite super-attractive de période $n$. Il donne dans [15] une image détaillée et partiellement conjecturelle de $\mathcal{S}_{n}$. Dans le présent article, nous démontrons ces conjectures pour $\mathcal{S}_{1}$ et généralisons ces résultats aux degrés supérieurs. En particulier, nous obtenons une description de la fermeture des composantes hyperboliques et des copies de Mandelbrot se trouvant dans le lieu de connexité. Nous prouvons que la frontière de chaque composante hyperbolique est une courbe de Jordan, dont les points sont caractérisés en fonction du comportement dynamique du polynôme associé. L'image globale du lieu de connexité est un disque fermé avec des "membres" qui en sortent aux cusps de copies de Mandelbrot et dont le diamètre tend vers 0 (ce qui correspond à une inégalité de Yoccoz quantitative).

(c) 2007 Elsevier Masson SAS

\section{Introduction}

In [4] Branner and Hubbard studied the parameter space of cubic polynomials (in terms of the dynamics). This space has been intensively studied since then. In this paper we focus on the 1-dimensional complex slice $\mathcal{S}_{1}$ of the cubic maps which have a fixed critical point. Our goal is to prove the conjectural picture given by Milnor in [14] of the connectedness locus in $\mathcal{S}_{1}$, and to generalize these results to degrees $d \geqslant 3$. The first question concerns the topology of the boundary of the main hyperbolic component. This leads naturally to the question of characterizing in dynamical terms the parameters on this boundary. The other problems are to describe first the intersections of the closures of hyperbolic components between each others and second their intersection with the Mandelbrot copies. Milnor considered also the limbs which

\footnotetext{
${ }^{1}$ Research partially supported by the Bernouilli Center in Lausanne. 


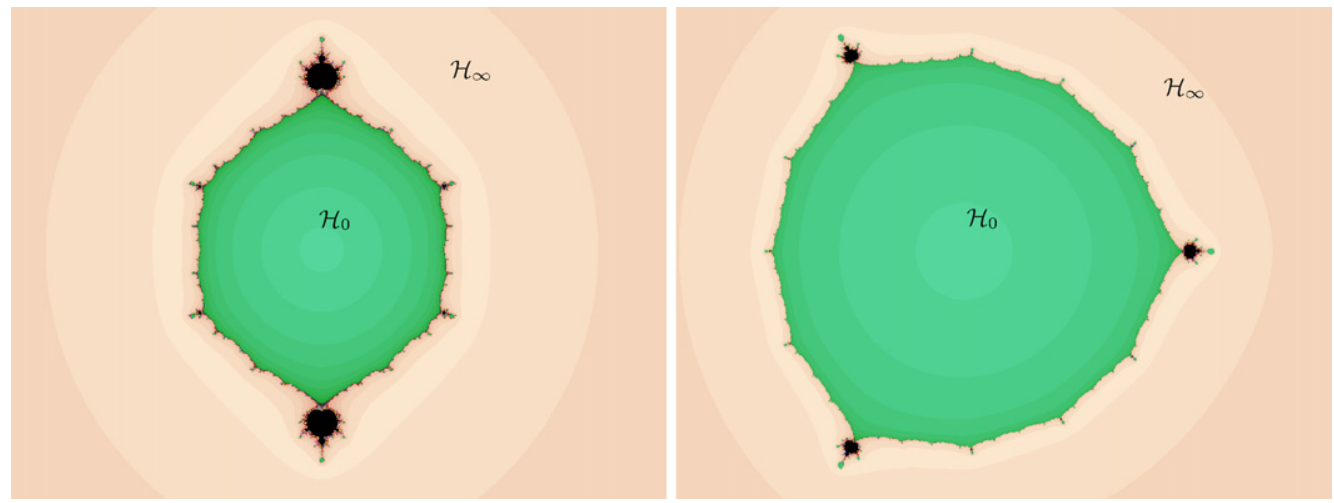

Fig. 1. Connectedness locus for $d=3$ and 4 in dark color.

are attached to the main hyperbolic component and raised the question of the existence of an analogue of Yoccoz' inequality, namely, the inequality which bounds the size of the limbs in the Mandelbrot case.

Let us consider the families of polynomials of degree $d \geqslant 3$ having a critical fixed point of maximal multiplicity. When we fix the degree, $d$, this set of polynomials is described-modulo affine conjugacy-by the following family $\left\{f_{\mathbf{a}}, \mathbf{a} \in \mathbf{C}\right\}$, where 0 is the critical fixed point of maximal multiplicity:

$$
f_{\mathbf{a}}(z)=z^{d-1}\left(z+\frac{d \mathbf{a}}{d-1}\right) .
$$

The set of parameters is partitioned into two loci: $\mathbf{C}=\mathcal{C} \sqcup \mathcal{H}_{\infty}$ (after [4]). The set $\mathcal{C}$ denotes the connectedness locus i.e., the set of parameters a such that the Julia set $J\left(f_{\mathbf{a}}\right)$ is connected; $\mathcal{H}_{\infty}$ consists of the parameters a such that the "free" critical point $-\mathbf{a}$ is attracted by $\infty$ (see [5]). We can continue the partition further, considering the hyperbolic parameters i.e., the parameters such that the orbit of every critical point converges to an attracting cycle (see [12,14]). This hyperbolic set is a disjoint union of open disks called hyperbolic components. The locus $\mathcal{H}_{\infty}$ is the unique unbounded hyperbolic component (see Lemma 1.9 or [14] and [4]). Among the hyperbolic components contained in $\mathcal{C}$, we focus on the ones associated to the attracting point 0 . The union of those is $\mathcal{H}=\left\{\mathbf{a} \in \mathbf{C} \mid-\mathbf{a} \in \widetilde{B}_{\mathbf{a}}\right\}$, where $\widetilde{B}_{\mathbf{a}}$ is the basin of attraction of the fixed point 0 .

\section{Local connectivity of the boundary of hyperbolic components}

THEOREM 1. - The boundary of every hyperbolic component of $\mathcal{C}$ is a Jordan curve.

This theorem is a consequence of Theorem 2 and the renormalization ${ }^{2}$ property of Proposition 1.

THEOREM 2. - The boundary of every connected component of $\mathcal{H}$ is a Jordan curve.

PROPOSITION 1. - If the map $f_{\mathbf{a}}$ has a non-repelling periodic point $p \neq 0$ then $f_{\mathbf{a}}$ is renormalizable near $p$ and the parameter $\mathbf{a}$ belongs to a copy of the Mandelbrot set $\mathbf{M}$.

Theorem 2 is the analogue in the parameter plane of the following dynamical result ${ }^{3}$ :

\footnotetext{
${ }^{2}$ Definitions of "renormalization" and of "copies of M" are given in Section 3.5.

${ }^{3}$ We will recall briefly the proof of it from Yoccoz' Theorem in Section 5.3.

$4^{\mathrm{e}}$ SÉRIE - TOME $40-2007-\mathrm{N}^{\circ} 6$
} 
THEOREM [7,17]. - The boundary of every connected component of $\widetilde{B}_{\mathbf{a}}$ is a Jordan curve.

Let us recall that D. Faught gave a proof of Theorem 2 in his thesis [7]. This result remains unpublished. For completeness, we give a proof of this result of local connectivity; our proof is different from that of [7], the argument here is based on an idea of Shishikura that simplifies the analysis.

Proposition 1 has the following two interesting corollaries:

COROLLARY 1. - Any hyperbolic component of $\mathcal{C}$ is either a connected component of $\mathcal{H}$ or a hyperbolic component of a copy of $\mathbf{M}$.

COROllary 2. - Assume that $f_{\mathbf{a}}$ possesses a periodic point $p$ with multiplier $\lambda=e^{2 i \pi \theta}$, such that $\theta \in \mathbf{R} \backslash \mathbf{Q}$. Then $f_{\mathbf{a}}$ is linearizable near $p$ if and only if $\theta \in \mathcal{B}$. Moreover, if $\theta \notin \mathcal{B}$ there exist periodic cycles in any neighbourhood of p.

Here $\mathcal{B}$ denotes the set of Brjuno numbers: an irrational $\theta$ of convergents $p_{n} / q_{n}$ (rational approximations obtained by the continued fraction development) is a Brjuno number, if $\sum_{n=1}^{\infty}\left(\log q_{n+1}\right) / q_{n}$ is finite.

\section{Parameters on the boundary of components of $\partial \mathcal{H}$}

Let $\mathcal{H}_{0}$ be the connected component of $\mathcal{H}$ containing 0 .

THEOREM 3. - Let $\mathbf{a} \in \partial \mathcal{H}_{0}$. There exists a unique parameter ray ${ }^{4}$ in $\mathcal{H}_{0}$ landing at $\mathbf{a}$, say $\mathcal{R}_{0}^{s}(t)$. The following dichotomy holds:

- there is a unique external parameter ray converging to a. In this case $f_{\mathbf{a}}$ is not renormalizable so a does not belong to a copy of $\mathbf{M}$. Moreover in the dynamical plane, the ray $R_{\mathbf{a}}^{0}(t)$ lands at the critical value $f_{\mathbf{a}}(-\mathbf{a}) \in \partial B_{\mathbf{a}}$ and there is a unique external ray converging to $f_{\mathbf{a}}(-\mathbf{a})$;

- there are exactly two external parameter rays converging to $\mathbf{a}$. In this case $\mathbf{a}$ is the cusp of a copy of $\mathbf{M}$. Furthermore, in the dynamical plane, the ray $R_{\mathbf{a}}^{0}(t)$ lands at a parabolic point on $\partial B_{\mathbf{a}}$. The angle $t$ is necessarily periodic by multiplication by $d-1$.

Proposition 2.32 gives a criterion on the angle $t$ to decide which one of the two cases described above arises.

THEOREM 4. - Let $\mathbf{a} \in \partial \mathcal{U}$ where $\mathcal{U} \neq \mathcal{H}_{0}$ is a connected component of $\mathcal{H}$. Then $\mathbf{a}$ is the landing point of a unique parameter ray of $\mathcal{U}$, say $\mathcal{R}_{\mathcal{U}}(t)$. In the dynamical plane, some iterate $f_{\mathbf{a}}^{k}(-\mathbf{a})$ lies in $\partial B_{\mathbf{a}}$ but $-\mathbf{a} \notin \partial B_{\mathbf{a}}$. Moreover, there exists a holomorphic function $r$, defined in a neighbourhood of $\mathbf{a}$, such that the dynamical ray $R_{\mathbf{a}}^{r(\mathbf{a})}(t)$ converges to the critical value $f_{\mathbf{a}}(-\mathbf{a})$. As a consequence, $f_{\mathbf{a}}$ has no parabolic cycles.

COROLLARY 3 (See also [8]). - For parameters a on the boundary of a component of $\mathcal{H}, f_{\mathbf{a}}$ cannot have an irrational indifferent periodic point.

\section{Intersections between the closures of hyperbolic components}

Lemma 1. - Any two distinct components of $\mathcal{H}$ have disjoint closures.

Recall that the cusp of $\mathbf{M}$ is the point $c=1 / 4$, and that the tips of $\mathbf{M}$ are the parameters $c \in \mathbf{M}$ such that $c$ falls after some iterations on the repelling fixed point, $\beta_{c}$ (the one that does

\footnotetext{
${ }^{4}$ Rays and parameter rays are defined in Sections 2 and 3.
} 


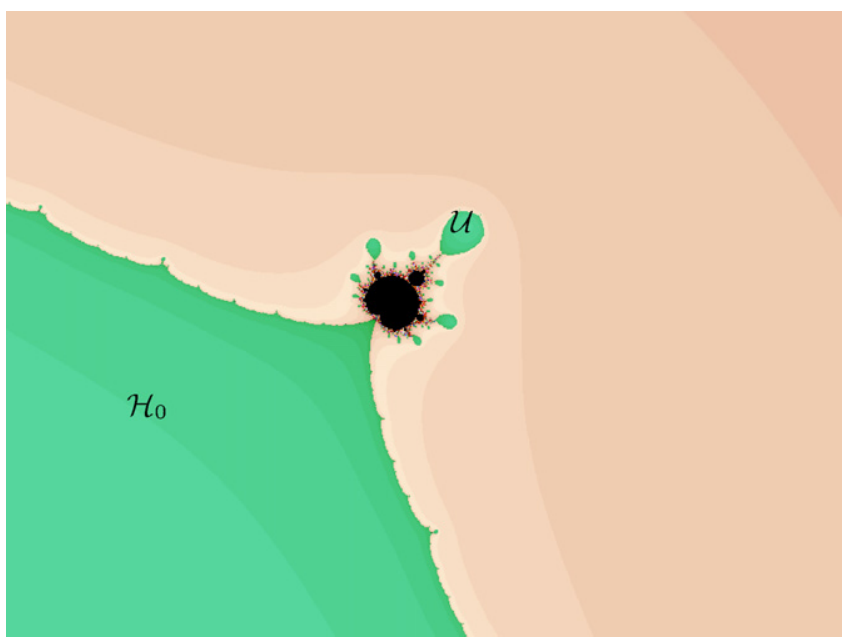

Fig. 2. A copy of $\mathbf{M}$ attached to $\mathcal{H}_{0}$ and a component $\mathcal{U}$ of $\mathcal{H} \backslash \mathcal{H}_{0}$.

not disconnect the Julia set). The cusp and the tips of a copy of $\mathbf{M}$ are the corresponding images by the homeomorphism defining the copy.

Let $\mathbf{M}_{0}$ be a copy of $\mathbf{M}$.

PRoposition 2. - If $\mathbf{M}_{0}$ intersects $\partial \mathcal{U}$ where $\mathcal{U}$ is a component of $\mathcal{H}$, the following dichotomy holds:

- if $\mathcal{U}=\mathcal{H}_{0}, \mathbf{M}_{0} \cap \partial \mathcal{U}$ is reduced to a single point, which is the cusp of $\mathbf{M}_{0}$;

- if $\mathcal{U} \neq \mathcal{H}_{0}, \mathbf{M}_{0} \cap \partial \mathcal{U}$ is reduced to a single point, which is a tip of $\mathbf{M}_{0}$. Furthermore, $\mathbf{M}_{0} \cap \partial \mathcal{H}_{0}$ is not empty, it reduces to the cusp of $\mathbf{M}_{0}$.

Conversely,

Proposition 3.- If $\mathbf{M}_{0}$ intersects $\partial \mathcal{H}_{0}$, then at any of its tips there is a connected component of $\mathcal{H} \backslash \mathcal{H}_{0}$ attached.

These results describe all the intersections between the boundaries of components of $\mathcal{H}$ and also with copies of $\mathbf{M}$, so in particular between all hyperbolic components of $\mathbf{C}$.

THEOREM 5. - The only intersections between closure of hyperbolic components, and also copies of $\mathbf{M}$ are the following:

- the central component $\mathcal{H}_{0}$ has Mandelbrot copies $\mathbf{M}_{t}$ attached to it at angles $t$ which are periodic by multiplication by $d-1$ (a full characterization of these values is given in Proposition 2.32);

- at every tip of such a satellite $\mathbf{M}_{t}$, there is a component $\mathcal{U}$ of $\mathcal{H} \backslash \mathcal{H}_{0}$ attached.

Nevertheless, there are infinitely many copies of $\mathbf{M}$ in $\mathcal{C}$ and components of $\mathcal{H}$ not in the category described above.

\section{Some global properties of $\mathcal{C}$}

THEOREM 6. - $\partial \mathcal{C}$ is locally connected at every point which is not in a copy of $\mathbf{M}$ and at any point of $\partial \mathcal{U}$ for every connected components $\mathcal{U}$ of $\mathcal{H}$.

$4^{\mathrm{e}}$ SÉRIE - TOME $40-2007-\mathrm{N}^{\circ} 6$ 
Concerning the $\operatorname{limbs} s^{5}$ of the main component $\mathcal{H}_{0}$, we obtain a qualitative version of Yoccoz' inequality for this family:

THEOREM 7. - For any $\epsilon>0$, only a finite number of limbs have diameter greater than $\epsilon$.

\section{Description of the content of the article}

In the first section we give some properties of the polynomials $f_{\mathbf{a}}$ in dynamical and parameter plane.

The second section is devoted to the parametrization of the components of $\mathcal{H}$ and also of $\mathcal{H}_{\infty}$. The parametrization is given by the Böttcher coordinate of the critical value and provides parameter rays and equipotentials.

In Section 3, we construct graphs that define puzzles to prove the local connectivity in the parameter space. They correspond-via the parametrization-to those used in the dynamical plane in [17] for the proof of the local connectivity of $\partial B_{\mathbf{a}}$ (we will recall the construction and the results of [17]). Then, the holomorphic motion of the dynamical graphs allows us to compare the puzzles in the parameter plane and in the dynamical plane, as pointed out by Shishikura in the case of quadratic polynomial (see [18]).

Section 4 is devoted to the proof of Theorem 1, 2, 3 and 4. Namely, we prove that when the intersection of the puzzle pieces (in the parameter plane) is not reduced to a single point, then this intersection is a copy of the Mandelbrot set.

In Section 5 we give the announced description of $\mathcal{C}$, i.e. the proof of Theorems 5, 6 and 7.

Finally, we add in Appendix A the proof of Theorem [7,17].

\section{Overview of dynamical and parameter plane}

Through the article we will take angles in $\mathbf{R} / \mathbf{Z}$ but in general we will have in mind their representant in $[0,1$. We will write $d t$ for the image of the angle $t \in \mathbf{R} / \mathbf{Z}$ by the multiplication by $d$ and $t / d$ for the element whose representative is in $[0,1 / d[$.

\subsection{The dynamics of $f_{\mathrm{a}}$}

We consider the polynomials $f_{\mathbf{a}}$ for a fixed degree $d \geqslant 3$. Note that for $d=2$ only the polynomial $P(z)=z^{2}$ satisfies the condition to have a super-attracting fixed point (modulo affine conjugacy).

Recall that the filled Julia set $K\left(f_{\mathbf{a}}\right)$ consists of the non-escaping points and that the Julia set $J_{\mathbf{a}}=J\left(f_{\mathbf{a}}\right)$ is its boundary:

$$
K_{\mathbf{a}}=K\left(f_{\mathbf{a}}\right)=\left\{z \mid f_{\mathbf{a}}^{n}(z) \underset{n \rightarrow \infty}{\longrightarrow} \infty\right\}, \quad J_{\mathbf{a}}=\partial K_{\mathbf{a}} .
$$

Note that for every $\mathbf{a} \in \mathbf{C}, K_{\mathbf{a}} \neq J_{\mathbf{a}}$ since $K_{\mathbf{a}}$ contains the basin of attraction of 0 , i.e.

$$
\widetilde{B}_{\mathbf{a}}=\left\{z \in \mathbf{C} \mid f_{\mathbf{a}}^{n}(z) \underset{n \rightarrow \infty}{\longrightarrow} 0\right\} .
$$

We denoted by $B_{\mathbf{a}}$ the immediate basin of 0 , that is the connected component of $\widetilde{B}_{\mathbf{a}}$ containing 0 . It follows from the maximum principle that $B_{\mathbf{a}}$ is a topological disc. If $-\mathbf{a} \notin B_{\mathbf{a}}$ the map $\left.f_{\mathbf{a}}\right|_{B_{\mathbf{a}}}$ is conjugated to $z^{d-1}$ on $\mathbf{D}$, else $B_{\mathbf{a}}=\widetilde{B}_{\mathbf{a}}$ (see [17] and the following Böttcher's theorem).

\footnotetext{
${ }^{5}$ Defined in Section 5.
} 
THEOREM (Böttcher). - For $p=0$ or $\infty$, there exist neighborhoods $V_{\mathbf{a}}^{p}, W_{\mathbf{a}}^{p}$ of $p$ such that $f_{\mathbf{a}}\left(V_{\mathbf{a}}^{p}\right) \subset V_{\mathbf{a}}^{p}$, and conformal isomorphisms $\phi_{\mathbf{a}}^{p}: V_{\mathbf{a}}^{p} \rightarrow W_{\mathbf{a}}^{p}$ satisfying

$$
\phi_{\mathbf{a}}^{\infty} \circ f_{\mathbf{a}}=\left(\phi_{\mathbf{a}}^{\infty}\right)^{d} \quad \text { on } \quad V_{\mathbf{a}}^{\infty} \quad \text { and } \quad \phi_{\mathbf{a}}^{0} \circ f_{\mathbf{a}}=\left(\phi_{\mathbf{a}}^{0}\right)^{d-1} \quad \text { on } \quad V_{\mathbf{a}}^{0} \quad(*)
$$

with $\phi_{\mathbf{a}}^{\infty}$ tangent to identity near $\infty$ and $\phi_{\mathbf{a}}^{0}$ tangent to $z \mapsto \lambda(\mathbf{a}) z$ near 0 where $\lambda(\mathbf{a})$ is a $(d-2)$-th root of $\frac{d \mathbf{a}}{d-1}$.

Remark 1.1. - The map $\phi_{\mathbf{a}}^{\infty}$ is always unique. Moreover, if we fix the choice of the $(d-2)$-th root $\lambda(\mathbf{a})$, the map $\phi_{\mathbf{a}}^{0}$ is also unique.

Assumption 1. - Through all the paper we will only consider parameters in $\mathbf{C} \backslash \mathbf{R}^{-}$(because of Remark 1.7). Thus, for the choice of $\lambda(\mathbf{a})$ we take the $(d-2)$-th principal root of $\frac{d \mathbf{a}}{d-1}$, i.e., the one such that $\lambda\left(\mathbf{R}^{+}\right) \subset \mathbf{R}^{+}$.

The Green function $G_{\mathbf{a}}^{\infty}$ (resp. $G_{\mathbf{a}}^{0}$ ) associated to $\infty$ (resp. to 0 ) is the harmonic map equal to $\log \left|\phi_{\mathbf{a}}^{\infty}(z)\right|$ on $V_{\mathbf{a}}^{\infty}$ (resp. to $-\log \left|\phi_{\mathbf{a}}^{0}(z)\right|$ on $V_{\mathbf{a}}^{0}$ ), extended on $\overline{\mathbf{C}} \backslash K_{\mathbf{a}}$ through the relation $d G_{\mathbf{a}}^{\infty}(z)=G_{\mathbf{a}}^{\infty}\left(f_{\mathbf{a}}(z)\right)$ (resp. on $\widetilde{B}_{\mathbf{a}}$ through $\left.(d-1) G_{\mathbf{a}}^{0}(z)=G_{\mathbf{a}}^{0}\left(f_{\mathbf{a}}(z)\right)\right)$ and vanishing on the complement.

DEFINITION 1.2. - The equipotential of level $v>0, E_{\mathbf{a}}^{p}(v)$, around $p=0$ or $\infty$ is the curve $E_{\mathbf{a}}^{p}(v)=\left\{z \in \mathbf{C} \mid G_{\mathbf{a}}^{p}(z)=v\right\}$. A ray, $R_{\mathbf{a}}^{p}(t)$, of angle $t \in \mathbf{R} / \mathbf{Z}$, stemming from $p=0$ or $\infty$, is a gradient line of $G_{\mathbf{a}}^{p}$ that coincides near $p$ with $\left(\phi_{\mathbf{a}}^{p}\right)^{-1}\left(\mathbf{R}^{+} e^{2 i \pi t}\right)$.

Note that if there is no critical point of $G_{\mathbf{a}}^{p}$ on a ray, it is a smooth simple curve; whereas at the critical point the gradient line divides itself so that several points have the same potential on this ray and also different points of $J_{\mathrm{a}}$ might be on the closure of a unique ray of given angle. This happens if $\mathbf{a} \in \mathcal{H}_{\infty} \cup \mathcal{H}_{0}$. In this case the angle $t$ and the potential do not define uniquely points on the ray, we will say that the ray is not well defined. In fact the ray is well defined in $\left\{z \mid G_{\mathbf{a}}^{p}(z)>G_{\mathbf{a}}^{p}(-\mathbf{a})\right\}$ since $\phi_{\mathbf{a}}^{p}$ extends to this set (via $(*)$ ). Note that it is possible to extend $\phi_{\mathbf{a}}^{0}$ continuously at the critical point $-\mathbf{a}$, but not $\phi_{\mathbf{a}}^{\infty}$ since there are two external rays crashing on $-\mathbf{a}$.

Finally, if a ray $R_{\mathbf{a}}^{p}(t)$ is well defined, it accumulates on the Julia set. We say that it lands if its accumulation set is reduced to one point and the landing point is in $J_{\mathbf{a}}$.

We have the following behaviour for rational rays (see $[5,12,16]$ ):

LEMma 1.3. - Let $\mathbf{a}_{0} \in \mathbf{C}, p=0$ or $\infty$, and $t \in \mathbf{Q} / \mathbf{Z}$. If the ray $R_{\mathbf{a}_{0}}^{p}(t)$ is well defined, it lands at an eventually periodic point which is repelling or parabolic. ${ }^{6}$

LEMMA 1.4. - Under the assumptions of Lemma 1.3, if the landing point is repelling and not eventually a critical point, there exists a neighbourhood $A$ of $\mathbf{a}_{0}$ such that for all $\mathbf{a} \in A$ the ray $R_{\mathbf{a}}^{p}(t)$ lands at a repelling point. Moreover, the map $(\mathbf{a}, s) \mapsto \psi_{\mathbf{a}, t}^{p}(s)$ is continuous on $A \times[0, \infty]$ and holomorphic in $\mathbf{a}$, where $\psi_{\mathbf{a}, t}^{p}(s)$ is the point on $R_{\mathbf{a}}^{p}(t)$ of potential $s$.

PROPOSITION 1.5 (Yoccoz). - For every eventually periodic point of $f_{\mathbf{a}}$ that is repelling or parabolic, there exists a rational angle $t$ such that $R_{\mathbf{a}}^{\infty}(t)$ lands at this point if $J\left(f_{\mathbf{a}}\right)$ is connected.

We will not need the analogue result in the non-connected case since we will start from the rays obtained when the Julia set is connected and proceeds to a holomorphic motion.

\footnotetext{
${ }^{6}$ A point $x$ of period $p$ is repelling, attracting, parabolic or indifferent irrational, respectively, if $\left|\left(f^{p}\right)^{\prime}(x)\right|>1$, $\left|\left(f^{p}\right)^{\prime}(x)\right|<1,\left(f^{p}\right)^{\prime}(x)=e^{2 i \pi \theta}$ and $\theta \in \mathbf{Q} / \mathbf{Z}$, or $\theta \in(\mathbf{R} \backslash \mathbf{Q}) / \mathbf{Z}$.

$4^{\mathrm{e}}$ SÉRIE - TOME $40-2007-\mathrm{N}^{\circ} 6$
} 


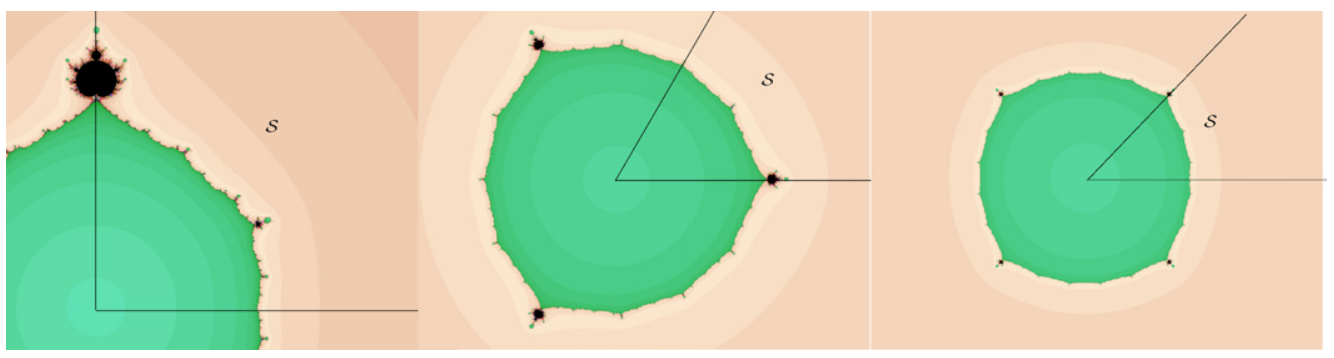

Fig. 3. Fundamental domain in degree $d=3,4$ and 5 .

LeMmA 1.6. - If two rays $R_{\mathbf{a}}^{0}(t)$ and $R_{\mathbf{a}}^{0}\left(t^{\prime}\right)$ land, their landing points are distinct when $t \neq t^{\prime}$.

Proof. - This follows, by a contradiction argument, from the maximum principle applied to the iterates of $f_{\mathbf{a}}$ on the domain bounded by the closed curve $\overline{R_{\mathbf{a}}^{0}(t)} \cup \overline{R_{\mathbf{a}}^{0}\left(t^{\prime}\right)}$.

\subsection{Parameter plane}

Remark 1.7. - The rotation $\tau(z)=\tau z$, where $\tau=e^{\frac{2 i \pi}{d-1}}$, is the only possible conformal conjugacy between polynomials $f_{\mathbf{a}}$ and $f_{\mathbf{a}^{\prime}}$; the relation is $f_{\tau \mathbf{a}}(\tau z)=\tau f_{\mathbf{a}}(z)$. Besides this, $f_{\mathbf{a}}$ is conjugated to $f_{\overline{\mathbf{a}}}$ by the complex conjugacy $\sigma(z)=\bar{z}$.

Hence a "fundamental domain" for the study of the family $f_{\mathbf{a}}$ is

$$
\mathcal{S}=\left\{\mathbf{a} \in \mathbf{C} \mid 0 \leqslant \arg (\mathbf{a}) \leqslant \frac{2 \pi}{2(d-1)}\right\}
$$

The connectedness locus $\mathcal{C}$, i.e., the set of parameters a such that $K_{\mathbf{a}}$ (or equivalently $J_{\mathbf{a}}$ ) is connected, admits the following classical characterization (see [5]):

$$
\mathcal{C}=\left\{\mathbf{a} \in \mathbf{C} \mid f_{\mathbf{a}}^{n}(-\mathbf{a}) \underset{n \rightarrow \infty}{\longrightarrow \rightarrow} \infty\right\} .
$$

Remark 1.8. - The sets $\mathcal{C}, \mathcal{H}$ and $\mathcal{H}_{\infty}$ admit $\sigma$ and $\tau$ as symmetries.

Lemma 1.9. - The set $\mathcal{H}_{\infty}=\mathbf{C} \backslash \mathcal{C}$ is a connected component of the set of hyperbolic parameters. Similarly, the connected component $\mathcal{H}_{0}$ is exactly the set $\left\{\mathbf{a} \mid-\mathbf{a} \in B_{\mathbf{a}}\right\}$.

Proof. - Clearly $\mathcal{H}_{\infty}$ contains a neighbourhood of $\infty$. So if $\mathcal{H}_{\infty}$ is not connected there is a bounded connected component, $\mathcal{U} \subset \mathcal{H}_{\infty}$. The boundary of $\mathcal{U}$ is in $\mathcal{C}$ so there exists some $M \in \mathbf{R}$ such that $\left|f_{\mathbf{a}}^{n}(-\mathbf{a})\right| \leqslant M$ for all $n \geqslant 0$ and $\mathbf{a} \in \partial \mathcal{U}$. For $\mathbf{a}_{0}$ a parameter in $\mathcal{U}$, there exists some $N$ such that $\left|f_{\mathbf{a}_{0}}^{n}\left(-\mathbf{a}_{0}\right)\right| \geqslant 2 M$ for $n \geqslant N$. This contradicts the maximum principle for the function $\mathbf{a} \mapsto f_{\mathbf{a}}^{N}(-\mathbf{a})$.

The proof for $\mathcal{H}_{0}$ goes with the same arguments exchanging 0 and $\infty$. Assume (by contradiction) that there is a connected component $\mathcal{U} \subset \mathbf{C}$ of $\left\{\mathbf{a} \mid-\mathbf{a} \in B_{\mathbf{a}}\right\}$ which is different from $\mathcal{H}_{0}$. There exists some $\epsilon>0$ such that on $\partial \mathcal{U},\left|f_{\mathbf{a}}^{n}(-\mathbf{a})\right|>\epsilon$ and for $\mathbf{a}_{0} \in \mathcal{U}$ there exists some $N$ such that $\left|f_{\mathbf{a}_{0}}^{n}\left(-\mathbf{a}_{0}\right)\right|<\epsilon / 2$ for $n \geqslant N$. This contradicts the maximum principle (as before) for the map $\mathbf{a} \mapsto 1 / f_{\mathbf{a}}^{N}(-\mathbf{a})$ which is well defined on a neighbourhood of $\overline{\mathcal{U}}$ since $f_{\mathbf{a}}^{N}(-\mathbf{a}) \neq 0$ for $\mathbf{a} \in \mathcal{U}$ because the sequence $f_{\mathbf{a}}^{n}(-\mathbf{a})$ tends geometrically to 0 in $B_{\mathbf{a}}$.

DEFINITION 1.10. - The so-called capture components of depth $i \geqslant 1$ are the connected components of $\mathcal{H}_{i}$, where $\mathcal{H}_{i}=\left\{\mathbf{a} \in \mathbf{C} \mid f_{\mathbf{a}}^{i}\left(f_{\mathbf{a}}(-\mathbf{a})\right) \in B_{\mathbf{a}}\right.$ and $\left.f^{i-1}\left(f_{\mathbf{a}}(-\mathbf{a})\right) \notin B_{\mathbf{a}}\right\}$. 
We have the following decomposition of the hyperbolic components of $\mathcal{H}$ :

Remark 1.11. $-\mathcal{H}=\bigcup_{i \geqslant 0} \mathcal{H}_{i}$.

Proof. - For $\mathbf{a} \in \mathcal{H}$, the critical point $-\mathbf{a}$ is attracted by 0 , so there exists $k \geqslant 0$ such that $f_{\mathbf{a}}^{k}(-\mathbf{a}) \in B_{\mathbf{a}}$. If $\mathbf{a} \notin \mathcal{H}_{i}$ for any $i \geqslant 0$, necessarily $f_{\mathbf{a}}(-\mathbf{a}) \in B_{\mathbf{a}}$ with $-\mathbf{a} \notin B_{\mathbf{a}}$. This is not possible since any point near $f_{\mathbf{a}}(-\mathbf{a})$ in $B_{\mathbf{a}}$ would have $d-1$ preimages in $B_{\mathbf{a}}$ (see Böttcher's Theorem) plus two near $-\mathbf{a}$ (the critical point), which exceeds the degree $d$ of $f_{\mathbf{a}}$.

A rough picture of the dynamics of $f_{\mathbf{a}}$ for $\mathbf{a} \in \mathcal{H} \cup \mathcal{H}_{\infty}$ is the following. For parameters a in $\mathcal{H}_{\infty}=\mathbf{C} \backslash \mathcal{C}$, the filled Julia set $K_{\mathrm{a}}$ is not connected but not totally disconnected since it contains the closed disc $\bar{B}_{\mathbf{a}}$. More precisely, $K_{\mathbf{a}}$ is the disjoint union of all the inverse images of $\bar{B}_{\mathbf{a}}$, the dynamics of $f_{\mathbf{a}}$ on $K_{\mathbf{a}}=\bigcup f_{\mathbf{a}}^{-i}\left(\bar{B}_{\mathbf{a}}\right)$ is the "shift" and on $\bar{B}_{\mathbf{a}}$ it is conjugated to $z \mapsto z^{d-1}$ on $\overline{\mathbf{D}}$.

For parameters a in $\mathcal{H}_{0}$ the critical point $-\mathbf{a}$ is in the immediate basin $B_{\mathbf{a}}$. Indeed, for the center $\mathbf{a}=0$ this is clear and the situation is stable. Thus for $\mathbf{a} \in \mathcal{H}_{0}$, the dynamics is very simple: $K_{\mathbf{a}}=\bar{B}_{\mathbf{a}}, B_{\mathbf{a}}=\widetilde{B}_{\mathbf{a}}, J_{\mathbf{a}}$ is a quasi-circle and $\left.f_{\mathbf{a}}\right|_{J_{\mathbf{a}}}$ is quasi-conformally conjugated to $z \mapsto z^{d}$ on $\mathbf{S}^{1}$.

For parameters $\mathbf{a} \in \mathcal{H}_{i}$ with $i \geqslant 1, K_{\mathbf{a}}=\bigcup_{j \geqslant 0} f_{\mathbf{a}}^{-j}\left(\bar{B}_{\mathbf{a}}\right)$, the map $f_{\mathbf{a}}$ is conjugated to $z \mapsto z^{d-1}$ on $\bar{B}_{\mathbf{a}}$ and corresponds to the "shift" on the components of $\left(f_{\mathbf{a}}^{-j}\left(\bar{B}_{\mathbf{a}}\right)\right)_{j \geqslant 0}$ not containing the critical point.

LEMMA 1.12. - Any connected component of $\mathcal{H}$, as well as $\mathcal{H}_{\infty} \cup\{\infty\}$, is simply connected.

Proof.-Once more this is an application of the maximum principle. The proof goes by contradiction. For the sake of simplicity we give the proof for a connected component $\mathcal{U}$ of $\mathcal{H}_{n}$ with $n \geqslant 0$. The argument follows for $\mathcal{H}_{\infty} \cup\{\infty\}$ by exchanging 0 and $\infty$.

Assume for contradiction that there exists a bounded connected component $K$ of $\mathbf{C} \backslash \mathcal{U}$. Then there exist points of $\partial \mathcal{U}$ in $K$ and also a simple closed curve $\gamma \subset \mathcal{U}$ surrounding $K$ since $\mathcal{U}$ is arcwise connected. In $\mathcal{U}$, the iterates of the critical point - a converge to 0 . Thus, for every $\epsilon>0$, there exists an $N \geqslant n$ such that for every $j \geqslant N$ and every parameter $\mathbf{a} \in \gamma,\left|f_{\mathbf{a}}^{j}(-\mathbf{a})\right|<\epsilon$. Now let $x$ be a point in $K \cap \partial \mathcal{U}$. Since $x \notin \mathcal{H}$, there exists an $r>0$ (depending on $x$ ) such that the iterates $f_{x}^{j}(-x) \cap B(0, r)=\emptyset$ for every $j \geqslant 0$. Taking $\epsilon=r / 2$, this contradicts the maximum principle for the holomorphic function $g(z)=f_{z}^{N}(-z)$, on the bounded open set delimited by $\gamma$.

Notation 1.13. - Let $\Upsilon_{0}: \mathcal{H}_{0} \rightarrow \mathbf{D}$, resp. $\Upsilon_{\infty}: \mathcal{H}_{\infty} \rightarrow \mathbf{C} \backslash \overline{\mathbf{D}}$, be the conformal representation tangent to the identity at 0 , resp. at $\infty$.

Remark 1.14. - Then, for $p=0$ or $\infty, \Upsilon_{p}(\sigma \mathbf{a})=\sigma \Upsilon_{p}(\mathbf{a})$ and $\Upsilon_{p}(\tau \mathbf{a})=\tau \Upsilon_{p}(\mathbf{a})$ with $\tau=e^{\frac{2 i \pi}{d-1}}$ and $\sigma(\mathbf{a})=\overline{\mathbf{a}}$. In other words $\mathcal{H}_{p}$ admits $\sigma$ and $\tau$ as symmetries.

Proof. - Since $\mathcal{H}_{p}$ is invariant by the complex involution $\sigma$ and the rotation $\tau$ (see Remark 1.7), the maps $\sigma \Upsilon_{p}(\sigma z)$ and $\Upsilon_{p}(\tau z) / \tau$ are conformal representations of $\mathcal{H}_{p}$ onto $\mathbf{D}$, or $\mathbf{C} \backslash \overline{\mathbf{D}}$, which are tangent to the identity at 0 , or at $\infty$. Hence $\Upsilon_{p}(\sigma z)=\sigma \Upsilon_{p}(z)$ and $\Upsilon_{p}(\tau z)=\tau \Upsilon_{p}(z)$.

COROLlaRY 1.15. - Let $\rho=e^{\frac{i \pi}{d-1}} ;$ for any $k \in \mathbf{N}$ the line $\rho^{k} \mathbf{R}^{+}$cuts $\mathcal{H}_{0}$ and $\mathcal{H}_{\infty}$ under a connected set. As a consequence, $\Upsilon_{0}\left(\rho^{k} \mathbf{R}^{+}\right)=\rho^{k}\left[0,1\left[\right.\right.$ and $\left.\left.\Upsilon_{\infty}\left(\rho^{k} \mathbf{R}^{+}\right)=\rho^{k}\right] 1,+\infty\right]$.

This does not imply that $\mathbf{R}^{+}$crosses only $\mathcal{H}_{0}, \mathcal{H}_{\infty}$ and $\partial \mathcal{C}$ (see Corollary 2.27).

Proof. - Fix $p \in\{0, \infty\}$. By Remark 1.14, for $\mathbf{a} \in \mathbf{R}^{+}, \Upsilon_{p}(\mathbf{a}) \in \mathbf{R}^{+}$and $\Upsilon_{p}\left(\mathbf{a} \rho^{k}\right) \in \rho^{k} \mathbf{R}^{+}$ (since $\rho^{k} \mathbf{a}=\rho^{2 k} \sigma\left(\rho^{k} \sigma(\mathbf{a})\right)=\rho^{2 k} \sigma\left(\rho^{k} \mathbf{a}\right)$ for $\mathbf{a} \in \mathbf{R}$, where $\sigma$ denotes the complex conjugacy). To prove that $\mathbf{R}^{+} \cap \mathcal{H}_{p}$ is connected we apply the maximum principle to a loop that we construct now. Assume for contradiction that there exist $x_{0}<x<x_{1}$ with $x \notin \mathcal{H}, x_{0}, x_{1} \in \mathcal{H}_{p}$; then there

$4^{e}$ SÉRIE - TOME $40-2007-\mathrm{N}^{\circ} 6$ 
is a simple arc $\gamma_{0} \subset \mathcal{H}_{p}$ with endpoints $x_{0}$ and $x_{1}$ such that $\gamma_{0} \backslash\left\{x_{0}, x_{1}\right\}$ avoids $\mathbf{R}$ (otherwise we change $x_{0}$ and $x_{1}$ to new adapted points). The desired loop in $\mathcal{H}_{p}$ surrounding $x$ is $\gamma=\gamma_{0} \cup \sigma\left(\gamma_{0}\right)$ (obtained by adding the conjugate). Hence $\mathbf{R}^{+} \cap \mathcal{H}_{p}$ is connected by the same argument as in Lemma 1.12. Using the symmetries $\sigma$ and $\tau$ (Remark 1.14) we deduce that for $k \geqslant 1$, the set $\rho^{k} \mathbf{R}^{+} \cap \mathcal{H}_{p}$ is also connected.

The following lemma will be useful for describing the domains of parametrization of the $\mathcal{H}_{i}$ for $i \in \mathbf{N} \cup\{\infty\}$. It gives some symmetry properties of the rays. These properties are specific to the family under consideration.

Lemma 1.16. - Fix $p \in\{0, \infty\}$. If the parameters $\mathbf{a}$ and $\tau \mathbf{a}$ are in $\mathbf{C} \backslash \mathbf{R}^{-}$, the Böttcher maps are related by some constants $\kappa_{p}$ as follows: $\sigma\left(\phi_{\sigma(\mathbf{a})}^{p}(\sigma(z))\right)=\phi_{\mathbf{a}}^{p}(z)=\kappa_{p}(\mathbf{a}) \phi_{\tau \mathbf{a}}^{p}(\tau z)$, with $\kappa_{\infty}(\mathbf{a})=\tau^{-1}$ and $\kappa_{0}(\mathbf{a})=\frac{\lambda(\mathbf{a})}{\tau \lambda(\tau \mathbf{a})}$. Then the rays at parameters $\mathbf{a}, \tau \mathbf{a}$ and $\sigma(\mathbf{a})$ satisfy the following relations, where $t_{p}(\mathbf{a})=\arg \left(\kappa_{p}(\mathbf{a})\right)$ :

$$
R_{\sigma(\mathbf{a})}^{p}(t)=\sigma\left(R_{\mathbf{a}}^{p}(-t)\right) \quad \text { and } \quad R_{\tau \mathbf{a}}^{p}(t)=\tau R_{\mathbf{a}}^{p} t\left(t+t_{p}(\mathbf{a})\right) .
$$

Proof. - Since $\tau^{-1} f_{\tau \mathbf{a}}(\tau z)=f_{\mathbf{a}}(z)$, the map $\tau^{-1} \phi_{\tau \mathbf{a}}^{\infty}(\tau z)$ conjugates $f_{\mathbf{a}}$ to $z \mapsto z^{d}$ near $\infty$. Since it is tangent to identity at $\infty, \tau^{-1} \phi_{\tau \mathbf{a}}^{\infty}(\tau z)=\phi_{\mathbf{a}}^{\infty}(z)$. Applying the same argument to the maps $\phi_{\tau \mathbf{a}}^{0}(\tau z)$ and $\sigma\left(\phi_{\sigma(\mathbf{a})}^{p}(\sigma(z))\right)$, we obtain that $\phi_{\sigma(\mathbf{a})}^{\infty}(\sigma(z))=\sigma\left(\phi_{\mathbf{a}}^{\infty}(z)\right)$ and that $\kappa_{0}^{\prime}(\mathbf{a}) \sigma\left(\phi_{\sigma(\mathbf{a})}^{0}(\sigma(z))\right)=\phi_{\mathbf{a}}^{0}(z)=\kappa_{0}(\mathbf{a}) \phi_{\tau \mathbf{a}}^{0}(\tau z)$ where $\kappa_{0}(\mathbf{a})$ and $\kappa_{0}^{\prime}(\mathbf{a})$ are appropriate constants. Taking the derivatives at 0 , we obtain $\kappa_{0}^{\prime}(\mathbf{a}) \sigma(\lambda(\sigma(\mathbf{a})))=\lambda(\mathbf{a})=\kappa_{0}(\mathbf{a}) \tau \lambda(\tau \mathbf{a})$. Note that $\kappa_{0}^{\prime}(\mathbf{a})=1$ since $\lambda=\sigma \circ \lambda \circ \sigma$.

Notation 1.17. - Let $\mathcal{S}^{+}$denote the connected component of $\mathbf{C} \backslash\left(\tau^{-1} \mathbf{R}^{-} \cup \mathbf{R}^{-}\right)$ containing $\mathbf{R}^{+}$for $d>3$, and $\mathcal{S}^{+}=\{z \mid \Im m(z)<0\}$ for $d=3$. Note that $\mathcal{S} \subset \mathcal{S}^{+}$.

Remark 1.18. - If a belongs to $\mathcal{S}^{+}$, then $\kappa_{0}(\mathbf{a})=\frac{1}{\tau \lambda(\tau)}=e^{\frac{-2 i \pi}{d-2}}$ and thus $t_{0}(\mathbf{a})=-\frac{1}{d-2}$.

\section{Coordinates in the parameter plane}

The conformal representations $\Upsilon_{0}$ and $\Upsilon_{\infty}$ are "a priori" independent of the dynamics. In this section we define a dynamical parametrization of $\mathcal{H} \cup \mathcal{H}_{\infty}$ as well as parameter rays and equipotentials.

\section{1. "Dynamical" parametrization of $\mathcal{H}_{0}$ and $\mathcal{H}_{\infty}$}

As usual, this parametrization is given by the "position" of the critical value. It is not defined everywhere, but can be extended by symmetry (see also [14]).

PROPOSITION 2.1. - The following map is a holomorphic covering of degree $d$ :

$$
\Phi_{\infty}:\left\{\begin{aligned}
& \mathcal{H}_{\infty} \longrightarrow \mathbf{C} \backslash \overline{\mathbf{D}} \\
& \mathbf{a} \longmapsto \Phi_{\infty}(\mathbf{a})=\phi_{\mathbf{a}}^{\infty}\left(f_{\mathbf{a}}(-\mathbf{a})\right)
\end{aligned}\right.
$$

Its restriction to $\mathcal{H}_{\infty} \cap \mathcal{S}$ is a homeomorphism onto $\Delta_{d}$ where

$$
\Delta_{d}=(-1)^{d-1}\left\{r e^{2 i \pi \theta} \mid r>1,0 \leqslant \theta \leqslant \frac{1}{2}+\frac{1}{2(d-1)}\right\} .
$$



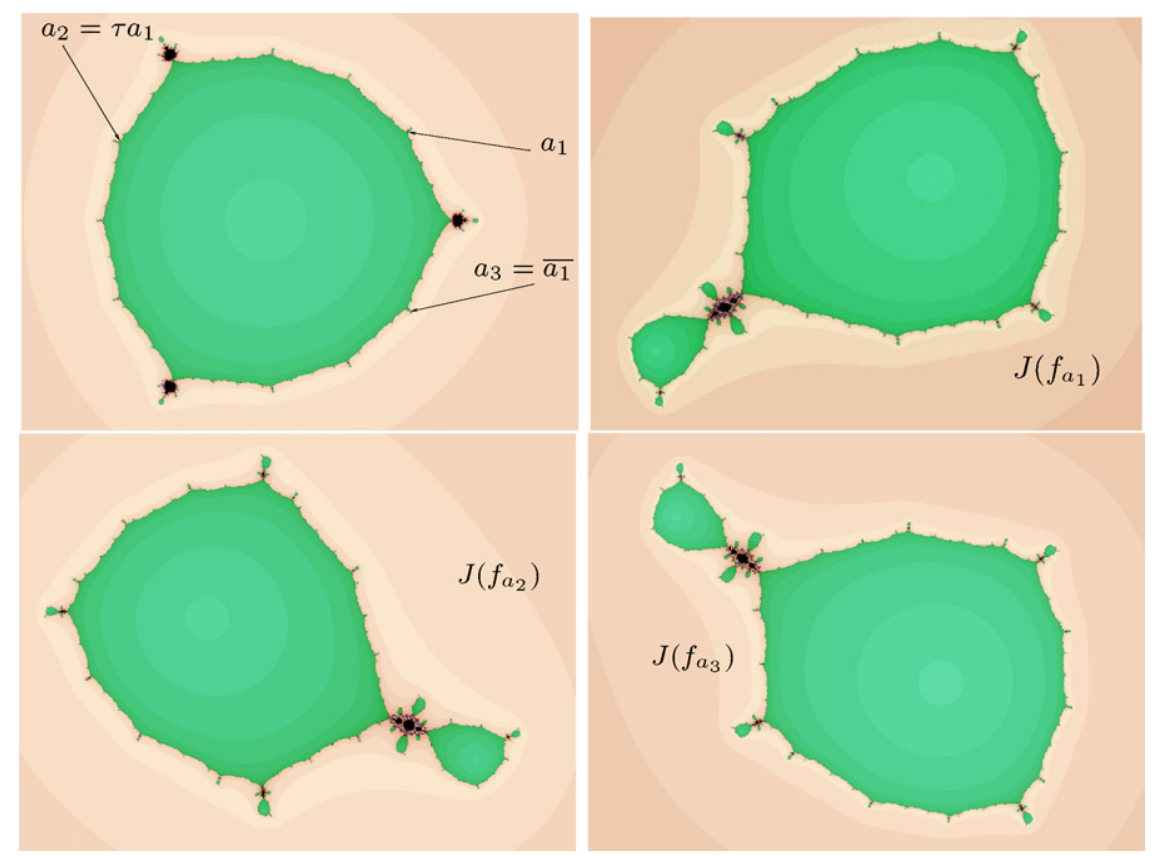

Fig. 4. Symmetric parameters $\mathbf{a}_{1}, \mathbf{a}_{2}, \mathbf{a}_{3}$ in $\mathcal{C}_{4}$ and $J\left(f_{\mathbf{a}_{1}}\right), J\left(f_{\mathbf{a}_{2}}\right), J\left(f_{\mathbf{a}_{3}}\right)$.

Proof. - The Böttcher coordinate $\phi_{\mathbf{a}}^{\infty}(z)$ is holomorphic in $(\mathbf{a}, z)$ where it is defined (see [2]) so $\Phi_{\infty}(\mathbf{a})$ is holomorphic on $\mathcal{H}_{\infty}$ with values in $\mathbf{C} \backslash \overline{\mathbf{D}}$. It extends by $\Phi_{\infty}(\infty)=\infty$ and $\Phi_{\infty}: \mathcal{H}_{\infty} \cup\{\infty\} \rightarrow \overline{\mathbf{C}} \backslash \overline{\mathbf{D}}$ is proper. Indeed, for any sequence $\mathbf{a}_{n} \in \mathcal{H}_{\infty}$ tending to $\mathbf{a} \in \partial \mathcal{C}$, the modulus $\left|\Phi_{\infty}\left(\mathbf{a}_{n}\right)\right|=e^{-G_{\mathbf{a}_{n}}^{\infty}\left(f_{\mathbf{a}_{n}}\left(-\mathbf{a}_{n}\right)\right)}$ tends to $e^{-G_{\mathbf{a}}^{\infty}\left(f_{\mathbf{a}}(-\mathbf{a})\right)}$ since the map $(\mathbf{a}, x) \mapsto G_{\mathbf{a}}^{\infty}(x)$ is continuous on $(\mathbf{C} \backslash\{0\}) \times \mathbf{C}$. Moreover, $G_{\mathbf{a}}^{\infty}\left(f_{\mathbf{a}}(-\mathbf{a})\right)=0$ since $f_{\mathbf{a}}(-\mathbf{a}) \in K_{\mathbf{a}}$ for $\mathbf{a} \in \mathcal{C}$, so $\left|\Phi_{\infty}\left(\mathbf{a}_{n}\right)\right| \rightarrow 1$.

The map $\Phi_{\infty}: \mathcal{H}_{\infty} \cup\{\infty\} \rightarrow \overline{\mathbf{C}} \backslash \overline{\mathbf{D}}$ is holomorphic and proper, hence it is a ramified covering whose degree is determined by the number of preimages of $\infty$. Since $\infty$ is its sole preimage we have to check the local degree at $\infty$. The following computations show that $\Phi_{\infty}(\mathbf{a}) \sim \frac{-(-\mathbf{a})^{d}}{d-1}$ at $\infty$, so that the degree is $d$. The function $F_{\mathbf{a}}(z)=f_{\mathbf{a}}(z) / z^{d}=1+\frac{d \mathbf{a}}{(d-1) z}$ is greater than $1 / 2$ for $z \in D_{\mathbf{a}}=\left\{\left.z|| z|>| \mathbf{a}\right|^{2}\right\}$ and large a. Thus $\left|f_{\mathbf{a}}(z)\right| \geqslant \frac{1}{2}|z|^{d} \geqslant|z|^{2}$ and $f_{\mathbf{a}}$ sends $D_{\mathbf{a}}$ into itself. Hence, since $F_{\mathbf{a}}\left(D_{\mathbf{a}}\right)$ is a small neighbourhood of 1 , for every $k \geqslant 1$, the quantity $\left(F_{\mathbf{a}}\left(f_{\mathbf{a}}^{k}(z)\right)\right)^{\frac{1}{d^{k+1}}}$ is well-defined on $D_{\mathbf{a}}$ by taking the principal determination (since $\phi_{\mathbf{a}}^{\infty}(z)$ is tangent to identity at $\infty$ ). The Böttcher coordinate $\phi_{\mathbf{a}}^{\infty}$ is the limit of the functions

$$
\phi_{\mathbf{a}, k}^{\infty}(z)\left(f_{\mathbf{a}}^{k}(z)\right)^{\frac{1}{d^{k}}}=z\left(F_{\mathbf{a}}(z)\right)^{\frac{1}{d}}\left(F_{\mathbf{a}}\left(f_{\mathbf{a}}(z)\right)\right)^{\frac{1}{d^{2}}} \cdots\left(F_{\mathbf{a}}\left(f_{\mathbf{a}}^{k}(z)\right)\right)^{\frac{1}{d^{k+1}}} .
$$

For $k \geqslant 1,\left|1-F_{\mathbf{a}}\left(f_{\mathbf{a}}^{k}(-\mathbf{a})\right)\right| \leqslant \frac{d}{d-1}|\mathbf{a}|^{1-2^{k}}$ since for large a the critical value $f_{\mathbf{a}}(-\mathbf{a})$ belongs to $D_{\mathbf{a}}$ and $\left|f_{\mathbf{a}}^{k}(-\mathbf{a})\right| \geqslant|\mathbf{a}|^{2^{k}}$. Thus,

$$
\sum_{k \geqslant 0} \frac{\log \left(F_{\mathbf{a}}\left(f_{\mathbf{a}}^{k+1}(-\mathbf{a})\right)\right)}{d^{k+1}} \underset{n \rightarrow \infty}{\nrightarrow} 0 \quad \text { and } \quad \Phi_{\infty}(\mathbf{a}) \sim_{\infty} f_{\mathbf{a}}(-\mathbf{a})=\frac{-(-\mathbf{a})^{d}}{d-1} .
$$



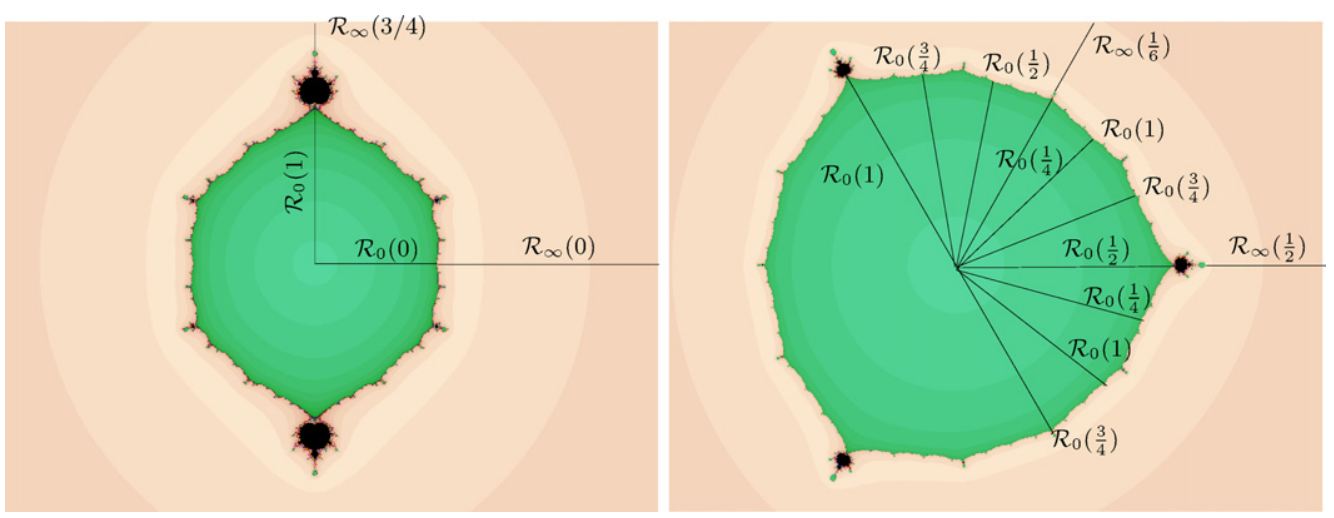

Fig. 5. Some rays in $\mathcal{H}_{0}$ and $\mathcal{H}_{\infty}$ for $d=3$ and $d=4$.

Hence $\Phi_{\infty}: \mathcal{H}_{\infty} \cup\{\infty\} \rightarrow \overline{\mathbf{C}} \backslash \overline{\mathbf{D}}$ is a covering of degree $d$ which is ramified only at $\infty$ (Riemann-Hurwitz formula). We can lift it through the covering $\overline{\mathbf{C}} \backslash \overline{\mathbf{D}} \stackrel{z \mapsto z^{d}}{\longrightarrow} \overline{\mathbf{C}} \backslash \overline{\mathbf{D}}$ to a map $v: \mathcal{H}_{\infty} \cup\{\infty\} \rightarrow \overline{\mathbf{C}} \backslash \overline{\mathbf{D}}$ satisfying $v(\mathbf{a})^{d} \sim \frac{-(-\mathbf{a})^{d}}{d-1}$ at $\infty$, so that we can choose $v$ tangent to $-\alpha e^{\frac{i \pi}{d}}$ Id at $\infty$ with $\alpha>0$. Therefore $v(\mathbf{a})=-e^{i \pi / d} \Upsilon_{\infty}(\mathbf{a}) /(d-1)^{1 / d}$ (they are conformal representations from $\mathcal{H}_{\infty} \cup\{\infty\}$ to $\overline{\mathbf{C}} \backslash \overline{\mathbf{D}}$ tangent at $\infty$, so they coincide). Hence

$$
\Phi_{\infty}(\mathbf{a})=(-1)^{d-1} \Upsilon_{\infty}(\mathbf{a})^{d} /(d-1) .
$$

This determines the image of $\mathcal{S} \cap \mathcal{H}_{\infty}$ by $\Phi_{\infty}$ using Corollary 1.15. Indeed, $\Phi_{\infty}\left(\mathbf{R}^{+} \cap \mathcal{H}_{\infty}\right)=$ $(-1)^{d-1} \mathbf{R}^{+} \backslash \overline{\mathbf{D}}$ and $\Phi_{\infty}\left(\rho \mathbf{R}^{+} \cap \mathcal{H}_{\infty}\right)=(-1)^{d} \rho \mathbf{R}^{+} \backslash \overline{\mathbf{D}}$ so that $\Phi_{\infty}\left(\mathcal{H}_{\infty} \cap \mathcal{S}\right)=\Delta_{d}$ since $\Phi_{\infty}$ preserves the cyclic order at $\infty$. Finally $\Phi_{\infty}$ is injective on $\mathcal{S} \cap \mathcal{H}_{\infty}$ because the opening of $\Upsilon_{\infty}(\mathcal{S})$ is less than $1 / d$ and $\Phi_{\infty}(\mathbf{a})=(-1)^{d-1} \Upsilon_{\infty}(\mathbf{a})^{d} /(d-1)$.

Remark 2.2. - For $d=3,4$ one has

$$
\Delta_{3}=\{] 1, \infty\left[e^{2 i \pi \theta} \mid 0 \leqslant \theta \leqslant \frac{3}{4}\right\}, \quad \Delta_{4}=\{] 1, \infty\left[e^{2 i \pi \theta} \mid \frac{1}{2} \leqslant \theta \leqslant \frac{1}{6}+1\right\} .
$$

Remark 2.3. - From the proof above and Remark 1.14, the following symmetries hold: $\Phi_{\infty}(\sigma(\mathbf{a}))=\sigma\left(\Phi_{\infty}(\mathbf{a})\right)$ and $\Phi_{\infty}(\tau \mathbf{a})=\tau \Phi_{\infty}(\mathbf{a})$ where $\tau=e^{\frac{2 i \pi}{d-1}}$.

PROPOSITION 2.4. - The map $\Phi_{0}(\mathbf{a})=\phi_{\mathbf{a}}^{0}\left(f_{\mathbf{a}}(-\mathbf{a})\right)$ is well-defined on $\mathcal{H}_{0} \backslash \mathbf{R}^{-}$.

- For $d>3$, its restriction to $\mathcal{H}_{0} \cap \mathcal{S}$ is a holomorphic homeomorphism onto

$$
\Lambda_{d}=(-1)^{d-1}\left\{r e^{2 i \pi \theta} \mid r \in\left[0,1\left[, 0 \leqslant \theta \leqslant \frac{1}{2}+\frac{1}{2(d-2)}\right\} .\right.\right.
$$

- For $d=3$, the restriction of $\Phi_{0}$ to $\mathcal{H}_{0} \cap \dot{\mathcal{S}}$ is a holomorphic homeomorphism onto $\mathbf{D} \backslash \mathbf{R}^{+}$, where $\dot{\mathcal{S}}$ denotes the interior of $\mathcal{S}$. Moreover, $\Phi_{0}$ maps bijectively each of the two boundary lines $\mathcal{H}_{0} \cap \mathbf{R}^{+}$and $\mathcal{H}_{0} \cap i \mathbf{R}^{+}$onto $\left[0,1\left[\right.\right.$ (with $\left.i^{2}=-1\right)$.

Proof. - The proof goes exactly as in Proposition 2.1; we just have to take care since $\phi_{\mathbf{a}}^{0}$ is not defined for $\mathbf{a} \in \mathbf{R}^{-}$(see Böttcher's Theorem, Section 1.1). We do it for $d>3$, the proof for $d=3$ follows the same arguments; the only difference is that the boundaries of $\Lambda_{d}$ glue together to give 
the disk for $d=3$, so if we forget the components of the boundary the arguments go through for $d=3$.

The map $\Phi_{0}$ is holomorphic on $\mathcal{H}_{0} \backslash \mathbf{R}^{-}$. Indeed, for $\mathbf{a} \in \mathcal{H}_{0} \backslash\left(\mathbf{R}^{-} \cup\{0\}\right) \Phi_{0}(\mathbf{a})=$ $\phi_{\mathbf{a}}\left(\lambda(\mathbf{a}) f_{\mathbf{a}}(-\mathbf{a})\right)$, where $\phi_{\mathbf{a}}$ is the Böttcher coordinate near 0 of the map $g_{\mathbf{a}}(z)=$ $\lambda(\mathbf{a}) f_{\mathbf{a}}(z / \lambda(\mathbf{a}))=z^{d-1}+\lambda(\mathbf{a})^{1-d} z^{d}$ (remark that $\lambda(\mathbf{a})$ is a non-vanishing holomorphic function on $\left.\mathcal{H}_{0} \backslash\left(\mathbf{R}^{-} \cup\{0\}\right)\right)$. As in Proposition 2.1 above, $\phi_{\mathbf{a}}$ is the limit of

$$
\phi_{\mathbf{a}, k}(z)=\left(g_{\mathbf{a}}^{k}(z)\right)^{\frac{1}{(d-1)^{k}}}=z\left(G_{\mathbf{a}}(z)\right)^{\frac{1}{d-1}} \cdots\left(G_{\mathbf{a}}\left(g_{\mathbf{a}}^{k}(z)\right)\right)^{\frac{1}{(d-1)^{k+1}}}
$$

where $G_{\mathbf{a}}(z)=g_{\mathbf{a}}(z) / z^{d-1}=1+\lambda(\mathbf{a})^{1-d} z$. The extension of $\phi_{\mathbf{a}}$, using $\phi_{\mathbf{a}} \circ g_{\mathbf{a}}=\left(\phi_{\mathbf{a}}\right)^{d-1}$ gives a holomorphic function $(\mathbf{a}, z) \rightarrow \phi_{\mathbf{a}}(z)$ with $\left(\phi_{\mathbf{a}}\right)^{\prime}(0)=1$.

It remains to prove that $\Phi_{0}$ is proper. As in the proof of Proposition 2.1, if $\mathbf{a} \in \mathcal{H}_{0} \backslash$ $\left(\mathbf{R}^{-} \cup\{0\}\right)$ tends to $\partial \mathcal{H}_{0}, \Phi_{0}(\mathbf{a})$ tends to $\partial \mathbf{D}$; an analogous computation shows that $\Phi_{0}(\mathbf{a}) \sim$ $-\lambda(\mathbf{a})(-\mathbf{a})^{d} /(d-1)$ near 0 . So we can extend $\Phi_{0}$ by $\Phi_{0}(0)=0$.

In order to determine the images of $\mathbf{R}^{+} \cap \mathcal{H}_{0}$ and $\rho \mathbf{R}^{+} \cap \mathcal{H}_{0}$, with $\rho=e^{i \pi /(d-1)}$, we can use that $\phi_{\mathbf{a}}^{0}$ is defined by continuity at the critical point $-\mathbf{a}$ and satisfies $\Phi_{0}(\mathbf{a})=$ $\phi_{\mathbf{a}}^{0}\left(f_{\mathbf{a}}(-\mathbf{a})\right)=\left(\phi_{\mathbf{a}}^{0}(-\mathbf{a})\right)^{d-1}$. From the proof of Remark 1.16, $\phi_{\mathbf{a}}^{0}\left(\mathbf{R}^{-}\right) \subset \mathbf{R}^{-}$for $\mathbf{a} \in \mathbf{R}^{+}$ since $\left(\phi_{\mathbf{a}}^{0}\right)^{\prime}(0) \in \mathbf{R}^{+}$and $\phi_{\mathbf{a}}^{0}(\sigma(z))=\sigma\left(\phi_{\mathbf{a}}^{0}(z)\right)$. Hence $\Phi_{0}\left(\mathbf{R}^{+}\right) \subset(-1)^{d-1} \mathbf{R}^{+}$. Now for $\mathbf{a} \in \rho \mathbf{R}^{+} \cap \mathcal{H}_{0}$, we determine $t$ such that $-\mathbf{a}$ "is" on the ray $R_{\mathbf{a}}^{0}(t)$, using the symmetries of Lemma 1.16 with $\tau=\rho^{2}$. Since $\tau \sigma(\mathbf{a})=\mathbf{a}$, the critical point $-\mathbf{a}$ is on $R_{\mathbf{a}}^{0}(t)=R_{\tau \sigma(\mathbf{a})}^{0}(t)$, thus $-\sigma(\mathbf{a})=-\tau^{-1} \mathbf{a} \in \tau^{-1} R_{\tau \sigma(\mathbf{a})}^{0}(t)$. Since $\tau^{-1} R_{\tau \sigma(\mathbf{a})}^{0}(t)=R_{\sigma \mathbf{a}}^{0}\left(t+t_{0}\right)=\sigma\left(R_{\mathbf{a}}^{0}\left(-t-t_{0}\right)\right)$, where $t_{0}=t_{0}(\sigma(\mathbf{a}))=\frac{-1}{d-2}$ (Remark 1.18), we deduce that the critical point $-\mathbf{a}$ is also on $R_{\mathbf{a}}^{0}\left(-t+\frac{1}{d-2}\right)$; so $t=-t+\frac{1}{d-2} \bmod 1$ because there is a unique ray stemming from 0 which contains the critical point (the case of bounded Fatou components). Hence, $2 t=\frac{1}{d-2} \bmod 1$ and the critical point belongs to $R_{\mathbf{a}}^{0}\left(\frac{1}{2(d-2)}\right)$ or to $R_{\mathbf{a}}^{0}\left(\frac{1}{2(d-2)}+\frac{1}{2}\right)$. The map $\phi_{\mathbf{a}}^{0}(z)$ is asymptotic to $\lambda(\mathbf{a}) z$ near 0 ; so any point $z \in \rho \mathbf{R}^{-}$near 0 is sent inside $e^{i \pi /(d-2)} \mathbf{R}^{-}$, since $\mathbf{a} \in \rho \mathbf{R}^{+}$. Finally, $\phi_{\mathbf{a}}^{0}(-\mathbf{a}) \in e^{i \pi /(d-2)} \mathbf{R}^{-}$and $\Phi_{0}(\mathbf{a}) \in(-1)^{d} e^{i \pi /(d-2)} \mathbf{R}^{+}$.

The image $\Phi_{0}\left(\mathcal{S} \cap \mathcal{H}_{0}\right)$ is exactly $\Lambda_{d}$. Indeed, the points of $\mathcal{S}$ near 0 are sent by $\Phi_{0}$ inside $\Lambda_{d}$. Moreover, if $\Phi_{0}\left(\mathcal{S} \cap \mathcal{H}_{0}\right)$ were bigger than $\Lambda_{d}$, there would be another connected component of $\Phi_{0}^{-1}\left(\partial \Lambda_{d}\right)$ in $\mathcal{S}$, but then $\Phi_{0}^{-1}(0) \cap \mathcal{S} \neq 0$ which is impossible $\left(\phi_{\mathbf{a}}^{0}\left(f_{\mathbf{a}}(-\mathbf{a})\right)=\right.$ $0 \Rightarrow \mathbf{a}=0$ ). Finally $\Phi_{0}$ is a proper holomorphic map from $\mathcal{S} \cap \mathcal{H}_{0}$ onto $\Lambda_{d}$. It is a ramified covering since $\mathcal{H}_{0} \cap \mathcal{S}$ is simply connected (by Lemma 1.12 and Corollary 1.15). The covering $\Phi_{0} \circ \Upsilon_{0}^{-1}: \Upsilon_{0}\left(\mathcal{S} \cap \mathcal{H}_{0}\right) \rightarrow \Lambda_{d}$ extends to all the boundary and its degree is the number of preimages of 0 . Thus $\Phi_{0}: \mathcal{S} \cap \mathcal{H}_{0} \rightarrow \Lambda_{d}$ is a holomorphic homeomorphism.

Remark 2.5. - From Lemma 1.16, Remark 1.18 and the proof above, the following symmetries hold: $\Phi_{0}(\sigma(\mathbf{a}))=\sigma\left(\Phi_{0}(\mathbf{a})\right)$ for $\mathbf{a} \notin \mathbf{R}^{-}$and, for $\mathbf{a} \in \mathcal{S}^{+}, \Phi_{0}(\tau \mathbf{a})=e^{\frac{2 i \pi}{d-2}} \Phi_{0}(\mathbf{a})$, where $\tau=e^{\frac{2 i \pi}{d-1}}$ and $\sigma(\mathbf{a})=\overline{\mathbf{a}}$.

Remark 2.6. - The difference (in the angles) between $\Lambda_{d}$ and $\Delta_{d}$ comes from the fact that the boundary of $\mathcal{H}_{\infty}$ will also touch the boundary of the components of $\mathcal{H}_{n}$ with $n>0$.

\subsection{Parametrization of $\mathcal{H}_{n}$}

LEMMA 2.7. - If $\mathcal{U}$ is a connected component of $\mathcal{H}_{n}$ with $n>0$, it cannot contain at the same time a point $\mathbf{a}_{0}$ and its symmetric $\tau \mathbf{a}_{0}$. Therefore, either $\mathcal{U}$ or $\tau \mathcal{U}$ is included in $\mathbf{C} \backslash \mathbf{R}^{-}$.

Proof. - Assume for contradiction that $\mathcal{U}$ contains a point $\mathbf{a}_{0}$ and its symmetric $\tau \mathbf{a}_{0}$. Then, we can construct a curve $\gamma$ surrounding 0 on which $f_{\mathbf{a}}^{n}$ is uniformly bounded for every $n: \gamma$ is

$4^{\text {e }}$ SÉRIE - TOME $40-2007-\mathrm{N}^{\circ} 6$ 
the union of an arc in $\mathcal{U}$ joining $\mathbf{a}_{0}$ to $\tau \mathbf{a}_{0}$ and all the symmetric images by $\tau^{k}$. This curve $\gamma$ is contained in $\mathcal{U}$ since $\mathcal{U} \cap \tau \mathcal{U} \neq \emptyset$, so that $\mathcal{U}=\tau \mathcal{U}=\cdots=\tau^{k} \mathcal{U}$. Then $\gamma$ surrounds $\overline{\mathcal{H}}_{0}$ since $0 \in \mathcal{H}_{0}$ and $\gamma \subset \mathcal{U} \neq \mathcal{H}_{0}$. This contradicts the fact that $\mathcal{U}$ is simply connected, Lemma 1.12.

Suppose now that $\mathcal{U}$ crosses $\mathbf{R}^{-}$and also $\tau^{-1} \mathbf{R}^{-}$; then it will necessary cross $\rho^{-1} \mathbf{R}^{-}$or $\rho \mathbf{R}^{-}$where $\rho=e^{i \pi / d-1}$. Moreover $\mathcal{U}=\sigma(\mathcal{U})$ since both have a common point on $\mathbf{R}^{-}$. Let $\mathbf{a}_{0}$ be some point of $\mathcal{U} \cap \rho^{-1} \mathbf{R}^{-}$. Then $\sigma\left(\mathbf{a}_{0}\right)$ belongs to $\mathcal{U}$. But $\sigma\left(\mathbf{a}_{0}\right)=\tau \mathbf{a}_{0}$ since $\tau \rho^{-1}=\sigma\left(\rho^{-1}\right)$. By the first part of this lemma, this is again a contradiction.

LEMMA 2.8. - Let $\mathcal{U}$ be a connected component of $\mathcal{H}_{n}$ with $n>0$ included in $\mathbf{C} \backslash \mathbf{R}^{-}$. The map

$$
\Phi_{\mathcal{U}}:\left\{\begin{array}{l}
\mathcal{U} \longrightarrow \mathbf{D} \\
\mathbf{a} \longmapsto \Phi_{\mathcal{U}}(\mathbf{a})=\phi_{\mathbf{a}}^{0}\left(f_{\mathbf{a}}^{n}\left(f_{\mathbf{a}}(-\mathbf{a})\right)\right)
\end{array}\right.
$$

is a conformal homeomorphism.

Proof. - For $\mathbf{a} \in \mathcal{U}$, the map $\phi_{\mathbf{a}}^{0}$ is well-defined, and is holomorphic in $(\mathbf{a}, z)$ for $z \in B_{\mathbf{a}}$ (see Remark 1.1). Hence since $\mathcal{U}$ is simply connected $\Phi_{\mathcal{U}}$ is a ramified covering from $\mathcal{U}$ to $\mathbf{D}$ (it is holomorphic and proper: the proof is similar to that of Proposition 2.4). It remains to show that it has degree one. For this we will prove that $\Phi_{\mathcal{U}}$ is a local homeomorphism near every point of $\mathcal{U}$. Let $\mathbf{a}_{0} \in \mathcal{U}$ and $z_{0}=\Phi_{\mathcal{U}}\left(\mathbf{a}_{0}\right)$. We will construct by surgery a local inverse $\mathbf{a}(z)$ to $z=\Phi_{\mathcal{U}}(\mathbf{a})$ in a neighbourhood of $z_{0}$. We first modify $f_{\mathbf{a}_{0}}$ near the point $f_{\mathbf{a}_{0}}^{n}\left(-\mathbf{a}_{0}\right)$, in order to send $f_{\mathbf{a}_{0}}^{n}\left(-\mathbf{a}_{0}\right)$ to $\left(\phi_{\mathbf{a}_{0}}^{0}\right)^{-1}(z)$ and we prove that this new map is quasi-conformally conjugated to $f_{\mathbf{a}(z)}$, so that $\Phi_{\mathcal{U}}(\mathbf{a}(z))=z$.

Let $B^{\prime}=f_{\mathbf{a}_{0}}^{-1}\left(B_{\mathbf{a}_{0}}\right), B^{\prime}$ is a topological disc. For $\epsilon$ small enough and for every $z \in D\left(z_{0}, \epsilon\right)$ one can construct a map $\delta_{z}: \overline{\mathbf{C}} \rightarrow \overline{\mathbf{C}}$ smooth in the variable $z$ and satisfying:

- $\delta_{z_{0}}=f_{\mathbf{a}_{0}}$,

- $\delta_{z}\left(f_{\mathbf{a}_{0}}^{n}\left(-\mathbf{a}_{0}\right)\right)=\left(\phi_{\mathbf{a}_{0}}^{0}\right)^{-1}(z)$,

- $\delta_{z}$ coincides with $f_{\mathbf{a}_{0}}$ outside $V$ which is a small neighbourhood of $f_{\mathbf{a}_{0}}^{n}\left(-\mathbf{a}_{0}\right)$ compactly included in $B^{\prime}$ (and independent of $z$ ),

- $\delta_{z}$ is a diffeomorphism from $B^{\prime}$ to $B_{\mathbf{a}_{0}}$.

We denote by $\sigma_{z}$ the complex structure which coincides with the standard complex structure on $B_{\mathbf{a}_{0}} \cup\left(\mathbf{C} \backslash \widetilde{B}_{\mathbf{a}_{0}}\right)$ and which is invariant by $\delta_{z}$. This complex structure has bounded dilatation. Let $g_{z}$ be the homeomorphism that integrates $\sigma_{z}$, given by Ahlfors-Bers' Theorem which is normalized to fix 0 and to be tangent to identity at $\infty$. Then the map $h_{z}=g_{z} \circ \delta_{z} \circ g_{z}^{-1}$ is holomorphic. Moreover it fixes $\infty$ with local degree $d$ and 0 with local degree $d-1$. Therefore, $h_{z}(u)=u^{d-1}(u+d \mathbf{a}(z) /(d-1))=f_{\mathbf{a}(z)}(u)$ with $\mathbf{a}(z)$ a continuous function of $z$ and $\mathbf{a}\left(z_{0}\right)=\mathbf{a}_{0}$. On $B_{\mathbf{a}_{0}}, g_{z}$ is holomorphic, conjugates $f_{\mathbf{a}_{0}}$ to $f_{\mathbf{a}(z)}$; so $\phi_{\mathbf{a}_{0}}^{0}$ and $\phi_{\mathbf{a}(z)}^{0} \circ g_{z}$ differ from a $d-2$ root of unity (since they both conjugate $f_{\mathbf{a}_{0}}$ to $z \mapsto z^{d-1}$ ). For $z=z_{0}$ this root of unity is 1 ; so, by continuity, $\phi_{\mathbf{a}_{0}}^{0}=\phi_{\mathbf{a}(z)}^{0} \circ g_{z}$. Moreover $g_{z}\left(-\mathbf{a}_{0}\right)=-\mathbf{a}(z)$ since $g_{z}$ preserves the critical points (looking at the local degree). This implies that $\Phi_{\mathcal{U}}(\mathbf{a}(z))=$ $\phi_{\mathbf{a}(z)}^{0}\left(f_{\mathbf{a}(z)}^{n+1}(-\mathbf{a}(z))\right)=z$ since $f_{\mathbf{a}(z)}^{n+1}(-\mathbf{a}(z))=g_{z} \circ \delta_{z}^{n+1} \circ g_{z}^{-1}(-\mathbf{a}(z))=g_{z} \circ \delta_{z}^{n+1}\left(-\mathbf{a}_{0}\right)=$ $g_{z} \circ \delta_{z}\left(f_{\mathbf{a}_{0}}^{n}\left(-\mathbf{a}_{0}\right)\right)$ and $g_{z} \circ \delta_{z}\left(f_{\mathbf{a}_{0}}^{n}\left(-\mathbf{a}_{0}\right)\right)=g_{z} \circ\left(\phi_{\mathbf{a}_{0}}^{0}\right)^{-1}(z)=\left(\phi_{\mathbf{a}(z)}^{0}\right)^{-1}(z)$.

Remark 2.9. - From Lemma 1.16 and Remark 1.18, the following symmetries hold: for $\mathbf{a} \in \mathcal{U}$, $\Phi_{\sigma(\mathcal{U})}(\sigma(\mathbf{a}))=\sigma\left(\Phi_{\mathcal{U}}(\mathbf{a})\right)$ and, $\Phi_{\tau \mathcal{U}}(\tau \mathbf{a})=e^{\frac{2 i \pi}{d-2}} \Phi_{\mathcal{U}}(\mathbf{a})$ if $\mathcal{U} \subset \mathcal{S}^{+}$, where $\tau=e^{\frac{2 i \pi}{d-1}}$.

\subsection{Parameter rays and equipotentials in $\mathbf{C} \backslash \mathbf{R}^{-}$}

DEFINITION 2.10. - We define the equipotential of level $v>0$, in $\mathcal{H}_{\infty}$ and in $\mathcal{H}_{0}$, by:

$$
\mathcal{E}_{\infty}(v)=\Phi_{\infty}^{-1}\left(\left\{e^{v+2 i \pi t}, t \in[0,1]\right\}\right), \quad \mathcal{E}_{0}(v)=\Phi_{0}^{-1}\left(\left\{e^{-v+2 i \pi t}, t \in[0,1]\right\}\right) .
$$


Note that an equipotential in $\mathcal{H}_{\infty}$ is a closed curve surrounding $\mathcal{C}$ and that an equipotential in $\mathcal{H}_{0}$ is never closed because the point in $\mathbf{R}^{-}$is missing. One can close it however by adding this point.

DEFINITION 2.11. - Let $p \in\{0, \infty\}$, we define the union of the rays of angle $t$ in $\mathcal{H}_{p}$, by

$$
\cup \mathcal{R}_{p}(t)=\left(\Phi_{p}\right)^{-1}\left(\mathbf{R}^{+} e^{2 i \pi t}\right) .
$$

Remark 2.12. - With this definition, there is no intersection between $\mathbf{R}^{-}$and $\cup \mathcal{R}_{0}(t)$ for any $t$.

DEFINITION 2.13. - Let $p \in\{0, \infty\}$ and $s \in\{\operatorname{Id}, \tau, \sigma, \tau \sigma, \ldots\}$ the result of any composition of the symmetries $\sigma$ and $\tau$. Denote by $\Phi_{p}^{s}$ the restriction to $s(\mathcal{S})$ of the map $\Phi_{p}$ and define the ray of angle $t$ in $\mathcal{H}_{p} \cap s(\mathcal{S})$ by

$$
\mathcal{R}_{p}^{s}(t)=\left(\Phi_{p}^{s}\right)^{-1}\left(\left\{r e^{2 i \pi t}, r \in \mathbf{R}^{+}\right\}\right)=\cup \mathcal{R}_{p}(t) \cap s(\mathcal{S}) .
$$

Remark 2.14.-

- The set $\mathcal{R}_{p}^{s}(t) \backslash\{0\}$ is connected except when $d=3, t=0$ and $s=\mathrm{Id}$;

- for $d=3$ and $t=0, \mathcal{R}_{0}^{\mathrm{Id}}(0) \backslash\{0\}$ has two connected components. Therefore we will make the convention that $\mathcal{R}_{0}^{\mathrm{Id}}(0)$ is the ray $\mathbf{R}^{+} \cap \mathcal{H}_{0}$ and $i \mathbf{R}^{+} \cap \mathcal{H}_{0}$ is the ray $\mathcal{R}_{0}^{\tau \sigma}(0)$;

- we will get rid of these notations when we will work in a para-puzzle piece $\mathcal{P}_{0}\left(\mathbf{a}_{0}\right)$ since from Remark 3.6, for every $t, \cup \mathcal{R}_{p}(t) \cap \mathcal{P}_{0}\left(\mathbf{a}_{0}\right)$ has only one connected component that we will call $\mathcal{R}_{p}(t)$.

LEMMA 2.15. - We have $\sigma\left(\mathcal{R}_{p}^{s}(t)\right)=\mathcal{R}_{p}^{\sigma s}(-t), \tau \mathcal{R}_{\infty}^{s}(t)=\mathcal{R}_{\infty}^{\tau s}\left(t+\frac{1}{d-1}\right)$ and if $\mathcal{R}_{0}^{s}(t) \subset$ $\mathcal{S}^{+}, \tau \mathcal{R}_{0}^{s}(t)=\mathcal{R}_{0}^{\tau s}\left(t+\frac{1}{d-2}\right)$.

Proof. - This follows from Remarks 2.5 and 2.3.

It follows from Lemma 2.15 that $\cup \mathcal{R}_{p}(t)$ is not invariant in general by $\sigma$ neither by $\tau$.

Remark 2.16. - We have the following correspondences for parameters $\mathbf{a} \in \mathbf{C} \backslash \mathbf{R}^{-}$and for $p \in\{0, \infty\}:\left(\mathbf{a} \in \cup \mathcal{R}_{p}(t) \Longleftrightarrow f_{\mathbf{a}}(-\mathbf{a}) \in R_{\mathbf{a}}^{p}(t)\right)$ and $\left(\mathbf{a} \in \mathcal{E}_{p}(v) \Longleftrightarrow f_{\mathbf{a}}(-\mathbf{a}) \in E_{\mathbf{a}}^{p}(v)\right)$.

Remark 2.17. - Let $p \in\{0, \infty\}$.

- The line $\mathbf{R}^{+} \cap \mathcal{H}_{p}$ is the ray $\mathcal{R}_{p}^{\mathrm{Id}}(0)$ if $d$ is odd, and $\mathcal{R}_{p}^{\mathrm{Id}}(1 / 2)$ if $d$ is even;

- the lines $\rho \mathbf{R}^{+} \cap \mathcal{H}_{0}$ and $\rho \mathbf{R}^{+} \cap \mathcal{H}_{\infty}$ are the rays $\mathcal{R}_{0}^{\mathrm{Id}}\left(\frac{1}{2}+\frac{1}{2(d-2)}\right)$ and $\mathcal{R}_{\infty}^{\mathrm{Id}}\left(\frac{1}{2}+\frac{1}{2(d-1)}\right)$ respectively if $d>3$ is odd, and $\mathcal{R}_{0}^{\mathrm{Id}}\left(\frac{1}{2(d-2)}\right)$ and $\mathcal{R}_{\infty}^{\mathrm{Id}}\left(\frac{1}{2(d-1)}\right)$ respectively if $d$ is even.

Proof. - This follows from Proposition 2.4 and Proposition 2.1 (those angles are given by the boundaries of $\Lambda_{d}$ and $\Delta_{d}$ ).

Definition 2.18. - Let $\mathcal{U} \subset \mathbf{C} \backslash \mathbf{R}^{-}$be a connected component of $\mathcal{H}_{n}$, with $n>0$. We define the center of $\mathcal{U}$ by $c_{\mathcal{U}}=\Phi_{\mathcal{U}}^{-1}(0)$ and the internal ray of angle $t \in \mathbf{R} / \mathbf{Z}$, resp. the equipotential of level $v$, by:

$$
\mathcal{R}_{\mathcal{U}}(t)=\Phi_{\mathcal{U}}^{-1}\left(\left\{r e^{2 i \pi t}, r<1\right\}\right), \quad \text { resp. } \quad \mathcal{E}_{\mathcal{U}}(v)=\Phi_{\mathcal{U}}^{-1}\left(\left\{e^{-v+2 i \pi t}, t \in[0,1]\right\}\right) .
$$

Note that $\mathcal{R}_{\mathcal{U}}(t) \backslash\left\{c_{\mathcal{U}}\right\}$ is connected since $\Phi_{\mathcal{U}}$ is a homeomorphism.

Remark 2.19. - For $\mathbf{a} \in \mathcal{H}_{n} \backslash \mathbf{R}^{-}$with $n>0$, the critical value $f_{\mathbf{a}}(-\mathbf{a})$ belongs to a connected component $U_{\mathbf{a}}$ of $\widetilde{B}_{\mathbf{a}}$. The map $f_{\mathbf{a}}^{n}: U_{\mathbf{a}} \rightarrow B_{\mathbf{a}}$ is a homeomorphism. Thus we can pull back the Böttcher coordinate to get coordinates on $U_{\mathbf{a}}$.

$4^{\text {e }}$ SÉRIE - TOME $40-2007-\mathrm{N}^{\circ} 6$ 
NOTATION 2.20. - We denote by $r$ and call the center of $U_{\mathbf{a}}$ the unique point of $U_{\mathbf{a}}$ which is sent by $f_{\mathbf{a}}^{n}$ to 0 (the center of $B_{\mathbf{a}}$ ). We denote by $R_{\mathbf{a}}^{r}(t)$ the ray stemming from $r$ with Böttcher coordinate $t$, i.e. the preimage $\left(\left.f_{\mathbf{a}}^{n}\right|_{U_{\mathbf{a}}}\right)^{-1}\left(R_{\mathbf{a}}^{0}(t)\right)$ which contains $r$ in its closure.

As in Lemma 1.6 we get now the criterion for connected components in the dynamical plane to have a common boundary point which is the landing point of rays.

LEMMA 2.21. - Let $\mathbf{a} \in \mathbf{C} \backslash \mathbf{R}^{-}$and $U, V$ be two connected components of $\widetilde{B}_{\mathbf{a}}$ with center $r, r^{\prime}$ respectively. If the rays $R_{\mathbf{a}}^{r}(t)$ and $R_{\mathbf{a}}^{r^{\prime}}\left(t^{\prime}\right)$ land at the same point $x$ then either $r=r^{\prime}$ and $t=t^{\prime}$ or the landing point $x$ is eventually critical.

Proof. - We assume that the rays $R_{\mathbf{a}}^{r}(t)$ and $R_{\mathbf{a}}^{r^{\prime}}\left(t^{\prime}\right)$ are different. After several iterations by $f_{\mathbf{a}}$, the image of the two rays in $B_{\mathbf{a}}$ should coincide by Lemma 1.6. Therefore we have, at some step of the iterations, the situation of two rays landing at a common point and having the same image under the map $f_{\mathbf{a}}$. Then the common landing point is the critical point $-\mathbf{a}$.

Note that when a is the center of a connected component $\mathcal{U}$ of $\mathcal{H}_{n}$, the critical value is the center of $U_{\mathbf{a}}$.

LEMMA 2.22. - Let $\mathcal{U}$ be a connected component of $\mathcal{H}_{n}$ with $n>0$. Let $\Omega$ be a simply connected neighbourhood of $\overline{\mathcal{U}}$ which avoids the centers of all the components of $\mathcal{H}_{j}$ with $j<n$. There exists on $\Omega$ a holomorphic map $r=r_{\mathcal{U}}: \Omega \rightarrow \mathbf{C}$ such that for $\mathbf{a} \in \mathcal{U}, r(\mathbf{a})$ is the center of the connected component that contains the critical value.

Proof. - We apply to $F(\mathbf{a}, z)=f_{\mathbf{a}}^{n}(z)$ the Implicit Function Theorem in a neighbourhood of the point $(\mathbf{a}, z)=\left(c_{\mathcal{U}}, f_{\mathcal{U}_{\mathcal{U}}}\left(-c_{\mathcal{U}}\right)\right)$. The only point where it is not possible to apply the theorem are the centers of $\mathcal{H}_{j}$ for $j<n$ since then the critical point is sent after $n$ iterations to 0 .

COROLLARY 2.23. - Let $\mathcal{U} \subset \mathbf{C} \backslash \mathbf{R}^{-}$be a connected component of $\mathcal{H}_{n}$ with $n>0$. We have the following equivalence: $\mathbf{a} \in \mathcal{R}_{\mathcal{U}}(t) \Longleftrightarrow f_{\mathbf{a}}(-\mathbf{a}) \in R_{\mathbf{a}}^{r(\mathbf{a})}(t)$.

\subsection{Landing properties}

Most of the results in this subsection follow as in the classical case of quadratic polynomials, see [5,12] and also [14]. Recall that for $\mathcal{U}=\mathcal{H}_{\infty}$ or $\mathcal{H}_{0}$, the ray $\mathcal{R}_{\mathcal{U}}^{s}(t)$ is the one in $s(\mathcal{S})$ where $s$ is any composition of $\sigma$ and $\tau$. Thus, it is not defined for any $t$; for instance for $s=\mathrm{Id}$, the angle has to be in $\Delta_{d}$ or in $\Lambda_{d}$. For a more detailed description and another proof of the following lemma, see Section 2.5.

Lemma 2.24. - Let $\mathcal{U} \subset \mathbf{C} \backslash \mathbf{R}^{-}$be a connected component of $\mathcal{H}_{n}$ with $n \in \mathbf{N} \cup\{\infty\}$. For $t$ rational, the ray $\mathcal{R}_{\mathcal{U}}^{s}(t)$ converges. Let $\mathbf{a}_{0}$ denote the landing point. If $\mathbf{a}_{0} \notin \mathbf{R}^{-}$, the ray $R_{\mathbf{a}_{0}}^{r\left(\mathbf{a}_{0}\right)}(t)$ is periodic (resp. eventually periodic) and lands at a parabolic periodic (resp. eventually periodic) point or at $f_{\mathbf{a}_{0}}\left(-\mathbf{a}_{0}\right)$ which is a repelling periodic (resp. eventually periodic) point.

In the last sentence, "eventually" depends on $t$, meaning that the number of iterates after which $R_{r\left(\mathbf{a}_{0}\right)}^{\mathbf{a}_{0}}(t)$ becomes periodic, and the period, both depend on $t$.

Proof. - Assume that $\mathcal{U}=\mathcal{H}_{\infty}$, the proof being easier for the other components. Let $\mathbf{a}_{0}$ be an accumulation point of $\mathcal{R}_{\infty}^{s}(t)$. Since $\mathbf{a}_{0} \in \mathcal{C}$ and $t \in \mathbf{Q}$ the ray $R_{\mathbf{a}_{0}}^{\infty}(t)$ is well-defined and converges. The landing point is (eventually) periodic, either parabolic or repelling. If it is repelling, and not eventually the critical value, by Lemma 1.3 we should have the stability of the rays $R_{\mathbf{a}_{0}}^{\infty}(t / d+k / d)$. But for parameters a on $\mathcal{R}_{\infty}^{s}(t)$ near $\mathbf{a}_{0}$ the critical value is on $R_{\mathbf{a}}^{\infty}(t)$ (by definition), so at least two of the previous rays crash on the critical point. So there exist 
$p, l$ depending only on $t$ such that $f_{\mathbf{a}_{0}}^{p+l}\left(-\mathbf{a}_{0}\right)=f_{\mathbf{a}_{0}}^{l}\left(-\mathbf{a}_{0}\right)$. If the landing point is eventually parabolic, the resultant of the two polynomials $f_{\mathbf{a}_{0}}^{p}(y)-y$ and $\left(f_{\mathbf{a}_{0}}^{p}\right)^{\prime}(y)-1$ vanishes since they have at least a common root. In both cases $\mathbf{a}_{0}$ is a root of a polynomial. Hence the accumulation set is a finite set and, since it is connected, it reduces to a point.

In the case where the landing point of $R_{\mathbf{a}_{0}}^{\infty}(t)$ is eventually repelling, let $k$ be the first integer such that the critical value lies on $\overline{R_{\mathbf{a}_{0}}^{\infty}\left(d^{k} t\right)}$. Thus the compact set $\overline{R_{\mathbf{a}_{0}}^{\infty}\left(d^{k} t\right)}$ moves continuously (by $\overline{R_{\mathbf{a}}^{\infty}\left(d^{k} t\right)}$ ) for a in a neighbourhood $\Omega$ of $\mathbf{a}_{0}$. In particular, for $\mathbf{a} \in\left(\mathcal{R}_{\infty}^{s}(t) \cup\left\{\mathbf{a}_{0}\right\}\right) \cap \Omega$, the compact set $\overline{R_{\mathbf{a}}^{\infty}(t)}$ is a continuous image of $\overline{R_{\mathbf{a}}^{\infty}\left(d^{k} t\right)}$ since $f_{\mathbf{a}}^{k}$ restricts on $R_{\mathbf{a}}^{\infty}(t)$ to a homeomorphism onto $R_{\mathbf{a}}^{\infty}\left(d^{k} t\right)$ (the holomorphic motion of the closure is obtained using the $\lambda$-Lemma). Therefore the critical value, which for $\mathbf{a} \in \mathcal{R}_{\infty}^{s}(t) \cap \Omega$ is in $R_{\mathbf{a}}^{\infty}(t)$, is for $\mathbf{a}=\mathbf{a}_{0}$ in $\overline{R_{\mathbf{a}_{0}}^{\infty}(t)}$. Therefore $R_{\mathbf{a}_{0}}^{\infty}(t)$ lands at $f_{\mathbf{a}_{0}}\left(-\mathbf{a}_{0}\right)$.

Definition 2.25. - A parameter a is Misiurewicz (or of Misiurewicz type) if for some $l \geqslant 1$, $z=f_{\mathbf{a}}^{l}(-\mathbf{a})$ is a periodic point of $f_{\mathbf{a}}$.

Note that if $f_{\mathbf{a}}^{l}(-\mathbf{a})$ is periodic (with $l \geqslant 1$ ), it is necessarily a repelling point. Indeed, if it is attracting or parabolic it would attract a critical point and there is no other "free" critical point that can converge to it. Note also that all the Misiurewicz points are in $\mathcal{C}$.

Lemma 2.26. - Let $\mathbf{a} \in \mathbf{C} \backslash \mathbf{R}^{-}$be a Misiurewicz point. There exists $t \in \mathbf{Q}$ such that $R_{\mathbf{a}}^{\infty}(t)$ lands at $f_{\mathbf{a}}(-\mathbf{a})$. Moreover, the ray $\mathcal{R}_{\infty}^{s}(t)$ lands at $\mathbf{a}$, for $s$ such that $\mathbf{a} \in s(\mathcal{S})$.

Proof. - The proof is exactly the same as in [5].

COROLLARY 2.27. - If $d$ is odd, $\mathbf{R}^{+}=\mathcal{R}_{\infty}^{\mathrm{Id}}(0) \cup \mathcal{R}_{0}^{\mathrm{Id}}(0) \cup\{*\}$ where $*$ is a Misiurewicz point.

Proof. - If $d=2 l+1, \mathbf{R}^{+} \cap \mathcal{H}_{0}=\mathcal{R}_{0}^{\text {Id }}(0)$ (Remark 2.17). Let $\mathbf{a}_{0}$ be the landing point of the ray $\mathcal{R}_{0}^{\text {Id }}(0), \mathbf{a}_{0} \in \mathbf{R}^{+*}$. The ray $R_{\mathbf{a}_{0}}^{0}(0) \subset \mathbf{R}^{+}$lands at a fixed point, say $x_{0} \in \mathbf{R}^{+*}$. If this fixed point is parabolic, the critical point $-\mathbf{a}_{0}$ is in a Fatou component attached to $x_{0}$. Thus, this Fatou component contains a curve which joins $-\mathbf{a}_{0}$ and $x_{0}$ and avoids 0 . By symmetry $(\sigma)$ this Fatou component contains a curve surrounding 0: this contradicts the fact that Fatou components are simply connected for polynomials. Therefore $x_{0}$ is repelling and $\mathbf{a}_{0}$ is a Misiurewicz parameter, so $x_{0}=f_{\mathbf{a}_{0}}\left(-\mathbf{a}_{0}\right)$ (Lemma 2.24).

The fixed ray $R_{\mathbf{a}_{0}}^{\infty}(0) \subset \mathbf{R}^{+}$also converges to a positive fixed point, say $x_{1}$. Assume that $x_{1} \neq x_{0}$. Then, from the shape of the graph of $\left.f_{\mathbf{a}_{0}}\right|_{\mathbf{R}^{+}}$, it is easy to see that since $x_{0}$ is repelling, either there are two other fixed points (one attracting and one repelling) or there is a parabolic fixed point of multiplier 1 . This implies that, including 0 , there are at least $d+1$ fixed points in $\mathbf{C}$ counted with multiplicity. This is not possible for a polynomial of degree $d$.

Therefore $x_{0}=x_{1}$ and the ray $R_{\mathbf{a}_{0}}^{\infty}(0)$ lands at $f_{\mathbf{a}_{0}}\left(-\mathbf{a}_{0}\right)$. So $\mathcal{R}_{\infty}^{\text {Id }}(0)$ lands at $\mathbf{a}_{0}$ (by Lemma 2.26).

Proposition 2.32 and Proposition 4.15 give the precise dynamical behaviour of $f_{\mathbf{a}}$ for parameters on $\partial \mathcal{H}_{0}$.

The parameters on $\mathbf{R}^{-}$excluded in all the results are obtained by symmetry.

LEMmA 2.28. - Two different rays in $\mathcal{U}$, where $\mathcal{U}$ is a connected component of $\mathcal{H}$, cannot converge to the same parameter.

Proof. - The proof is the same in any $\mathcal{H}_{i}$ so we do it for $\mathcal{U}=\mathcal{H}_{0}$. Assume, to get a contradiction, that two rays of $\mathcal{H}_{0}$ converge to the same point $\mathbf{a}_{0}$. One can suppose (up to changing the rays) that they belong to the same $s(\mathcal{S})$, so that it is enough to consider the case $s=\mathrm{Id}$. Let $\mathcal{R}_{0}(t)$ and $\mathcal{R}_{0}\left(t^{\prime}\right)$ be the two rays under consideration. Let $\gamma$ be the curve

$4^{\mathrm{e}}$ SÉRIE - TOME $40-2007-\mathrm{N}^{\circ} 6$ 
$\mathcal{R}_{0}(t) \cup \mathcal{R}_{0}\left(t^{\prime}\right) \cup\left\{\mathbf{a}_{0}\right\} \cup\{0\}$. There are infinitely many angles of the form $\frac{p}{q\left((d-1)^{k}-1\right)}$ between $t$ and $t^{\prime}$, and infinitely many of them give rays landing to Misiurewicz parameters. Indeed, for such an angle $\theta$, the ray $\mathcal{R}_{0}(\theta)$ converges to a parameter a which is either of Misiurewicz type or such that the map $f_{\mathbf{a}}$ has a parabolic point of period $k$ with multiplier 1 since it is the landing point of a ray in $B_{\mathbf{a}}$ (see Lemma 2.24). As there is only a finite number of parameters a satisfying the second alternative (they are solutions of a polynomial equation of degree at most $d^{k}$ ), we know that infinitely many of these landing parameters are Misiurewicz points (on $\partial \mathcal{H}_{0} \backslash\left\{\mathbf{a}_{0}\right\}$ ) lying in the bounded component of $\mathbf{C} \backslash \gamma$. This contradicts the fact that Misiurewicz parameters are landing points of external parameter rays (Lemma 2.26) because such external rays will have to cross $\gamma$ to enter the bounded component of $\mathbf{C} \backslash \gamma$.

\subsection{Description of the dynamical position of the critical value}

Note that in degree $d \geqslant 3$, the position of the critical value does not give directly the position of the critical point as in degree 2 . We will now give the Böttcher coordinate of the critical point for any parameter $\mathbf{a} \in \mathcal{S} \cap\left(\mathcal{H}_{\infty} \cup \mathcal{H}_{0}\right)$, so in this subsection we forget the exponent specifying the sector for the parameter rays.

LEMMA 2.29. - For $\mathbf{a} \in \mathcal{R}_{\infty}(t)$, the rays $R_{\mathbf{a}}^{\infty}\left(\frac{t}{d}+\frac{\left\lfloor\frac{d-1}{2}\right\rfloor}{d}\right), R_{\mathbf{a}}^{\infty}\left(\frac{t}{d}+\frac{\left\lfloor\frac{d+1}{2}\right\rfloor}{d}\right)$ crash on the critical point $-\mathbf{a}$. If $\mathbf{a} \in \mathcal{R}_{0}(t)$, the critical point $-\mathbf{a}$ belongs to $R_{\mathbf{a}}^{0}\left(\frac{t}{d-1}+\frac{\left\lfloor\frac{d-1}{2}\right\rfloor}{d-1}\right)$.

Proof. - For $\mathbf{a} \in \mathcal{R}_{\infty}(t), f_{\mathbf{a}}(-\mathbf{a})$ belongs to $R_{\mathbf{a}}^{\infty}(t)$, so the two rays crashing on $-\mathbf{a}$ belong to the set of rays $\rceil=\left\{R_{\mathbf{a}}^{\infty}\left(\frac{t}{d}+\frac{k}{d}\right) \mid 0 \leqslant k \leqslant d-1\right\}$. If $\mathbf{a} \in \mathcal{R}_{0}(t)$, the critical point $-\mathbf{a}$ belongs to a unique ray of the set $\beth=\left\{R_{\mathbf{a}}^{0}\left(\frac{t}{d-1}+\frac{k}{d-1}\right) \mid 0 \leqslant k \leqslant d-2\right\}$.

We first describe the case of parameters $\mathbf{a} \in \mathbf{R}^{+}$: this case is more visual because of the symmetry $R_{\mathbf{a}}^{p}(-\theta)=\sigma\left(R_{\mathbf{a}}^{p}(\theta)\right)$ (where $\sigma$ is the complex conjugacy). Then we conclude by moving a through $\mathcal{S}$.

1) For $0<\mathbf{a} \ll 1$, the critical point is on $R_{\mathbf{a}}^{0}\left(\frac{1}{2}\right) \subset \mathbf{R}^{-}$, since $\mathbf{a} \in \mathcal{H}_{0} \cap \mathbf{R}^{+}$. For $d=2 l+1$, $\mathbf{a} \in \mathcal{R}_{0}(0)$ so $t=0$; we verify then that $R_{\mathbf{a}}^{0}\left(\frac{1}{2}\right)=R_{\mathbf{a}}^{0}\left(0+\frac{l}{d-1}\right)$. For $d=2 l+2, \mathbf{a} \in \mathcal{R}_{0}\left(\frac{1}{2}\right)$, so $t=\frac{1}{2}$; we verify in that case that $R_{\mathbf{a}}^{0}\left(\frac{1}{2}\right)=R_{\mathbf{a}}^{0}\left(\frac{1}{2(d-1)}+\frac{l}{d-1}\right)$.

For $\mathbf{a} \in \mathcal{S} \cap \mathcal{H}_{0}, \phi_{\mathbf{a}}^{0}(-\mathbf{a})$ is well-defined and continuous, and so it belongs to $e^{2 i \pi\left(\frac{t}{d-1}+\frac{k}{d-1}\right)} \mathbf{R}^{+}$ for $\mathbf{a} \in \mathcal{R}_{0}(t)$. The integer $k$ is a continuous function of $\mathbf{a}$, so it is constant and therefore equal to $\left\lfloor\frac{d-1}{2}\right\rfloor$.

2) We consider now the case $\mathbf{a} \gg 1$. The fixed rays $R_{\mathbf{a}}^{0}\left(\frac{k}{d-2}\right), R_{\mathbf{a}}^{\infty}\left(\frac{k}{d-1}\right)$ with $k \in \mathbf{N}$ are well defined. Indeed, $-\mathbf{a} \notin K_{\mathbf{a}}$ so every rational ray in $B_{\mathbf{a}}$ converges. Moreover, the only fixed rays in 7 are $R_{\mathbf{a}}^{\infty}(0) \subset \mathbf{R}^{+}$and, if $d$ is odd, $\left.R_{\mathbf{a}}^{\infty}\left(\frac{1}{2}\right)=\right]-\infty, p[$ where $p$ is the unique negative fixed point of $f_{\mathbf{a}}$; note that $p<-\mathbf{a}$ since $f_{\mathbf{a}}(-\mathbf{a})>0$. By Lemma 1.6, distinct internal rays of $R_{\mathbf{a}}^{0}\left( \pm \frac{k}{d-2}\right)$ $(k \in \mathbf{N})$ converge to distinct fixed points, named $x_{ \pm k}$. Those $x_{k}$ are repelling points $(\mathbf{a} \notin \mathcal{C})$. They are the landing points of external rays (see [10]), which are also fixed rays because of the rotation number at $x_{k}$ (see also [16]). Those rays belong to $\left\{R_{\mathbf{a}}^{\infty}\left(\frac{p}{d-1}\right), 0 \leqslant p \leqslant d-2\right\}$. Because of the symmetry, $R_{\mathbf{a}}^{\infty}\left(\frac{ \pm k}{d-1}\right)$ and $R_{\mathbf{a}}^{0}\left(\frac{ \pm k}{d-2}\right)$ converge to $x_{ \pm k}$ for $0 \leqslant k<\left\lfloor\frac{d}{2}\right\rfloor$. Thus, for $l=\left\lfloor\frac{d-1}{2}\right\rfloor$, $\gamma_{\mathbf{a}}=\overline{R_{\mathbf{a}}^{\infty}}\left( \pm \frac{l-1}{d-1}\right) \cup \overline{R_{\mathbf{a}}^{0}}\left( \pm \frac{l-1}{d-2}\right)$ is a curve "separating" $\mathbf{C}$ into two connected components; let $U_{\mathbf{a}}$ be the one which contains $-\mathbf{a}$. The only rays of 7 entering $U_{\mathbf{a}}$ are $R_{\mathbf{a}}^{\infty}\left(\frac{d-1}{2 d}\right), R_{\mathbf{a}}^{\infty}\left(\frac{d+1}{2 d}\right)$; so they crash on $-\mathbf{a}$ for $\mathbf{a} \gg 1$.

By Lemma 1.4, $\gamma_{\mathbf{a}}$ admits a holomorphic motion parameterized by $\mathcal{S} \cap \mathcal{H}_{\infty}$. Indeed, if $-\mathbf{a}$ belongs to $\gamma_{\mathbf{a}}$ the critical value would describe on the external rays a sector of opening more than $\frac{d \pi}{d-1}$ which is impossible since it is exactly the opening of $\Phi_{\infty}(\mathcal{S})$. Therefore $-\mathbf{a}$ stays in 

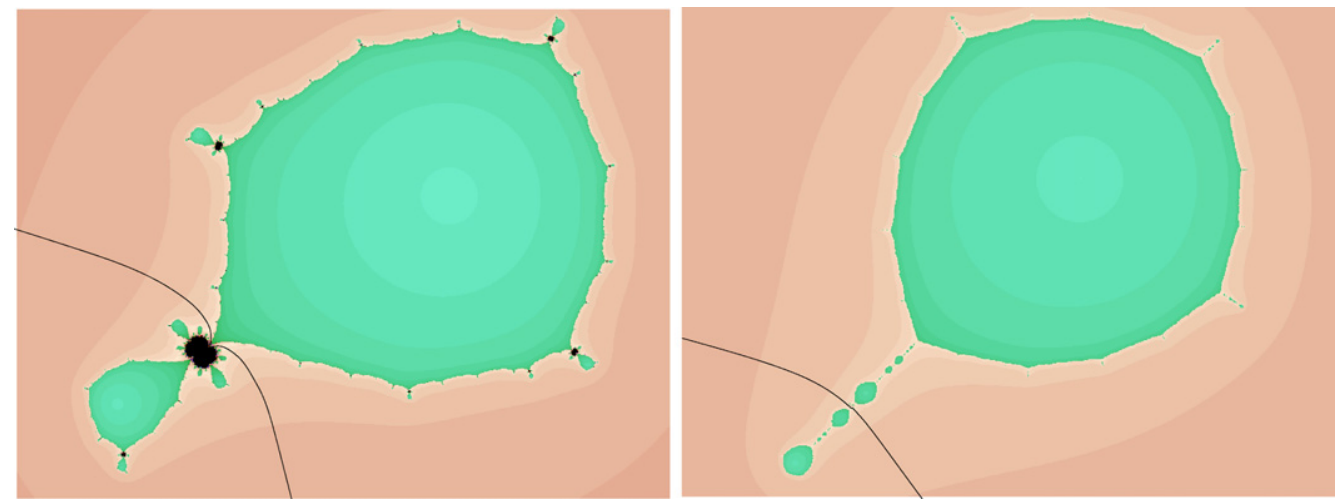

Fig. 6. Two rays converging to the parabolic point (on the left), to the critical point (on the right) for some parameters in $\mathcal{C}_{4}$.

$U_{\mathbf{a}}$ and the only candidates of $\rceil$ in $U_{\mathbf{a}}$ are $R_{\mathbf{a}}^{\infty}\left(\frac{t}{d}+\frac{\left\lfloor\frac{d-1}{2}\right\rfloor}{d}\right)$ and $R_{\mathbf{a}}^{\infty}\left(\frac{t}{d}+\frac{\left\lfloor\frac{d+1}{2}\right\rfloor}{d}\right)$, so they crash on $-\mathbf{a}$.

COROLlaRY 2.30. - For $\mathbf{a} \in \mathcal{S}$, if the critical point $-\mathbf{a}$ belongs to $R_{\mathbf{a}}^{\infty}(\theta)$ then $\theta \in\left[\frac{1}{2}-\frac{1}{d}\right.$, $\left.\frac{1}{2}+\frac{1}{d}\right]$, if $-\mathbf{a} \in R_{\mathbf{a}}^{0}(\theta)$ then $\theta \in\left[\frac{1}{2}-\frac{1}{2(d-1)}, \frac{1}{2}+\frac{1}{d-1}\right]$.

Proposition 2.31. - For $t \in \mathbf{Q}$, the ray $\mathcal{R}_{\infty}(t)$ converges to a parameter $\mathbf{a}_{\infty}(t)$. For $l=\left\lfloor\frac{d-1}{2}\right\rfloor:$

1. if $\frac{t}{d}+\frac{l}{d}$ (resp. $\frac{t}{d}+\frac{l+1}{d}$ ) is periodic by multiplication by $d, \mathbf{a}_{\infty}(t)$ is a parabolic parameter. The ray $R_{\mathbf{a}_{\infty}(t)}^{\infty}\left(\frac{t}{d}+\frac{l}{d}\right)$ (resp. $R_{\mathbf{a}_{\infty}(t)}^{\infty}\left(\frac{t}{d}+\frac{l+1}{d}\right)$ ) lands at a parabolic point $p$, root of the Fatou component $P_{t}$ containing the critical point $-\mathbf{a}_{\infty}(t)$ and the ray $R_{\mathbf{a}_{\infty}(t)}^{\infty}\left(\frac{t}{d}+\frac{l+1}{d}\right)$ (resp. $\left.R_{\mathbf{a}_{\infty}(t)}^{\infty}\left(\frac{t}{d}+\frac{l}{d}\right)\right)$ lands at the preimage of $f_{\mathbf{a}_{\infty}(t)}(p)$ on $\partial P_{t}$;

2. otherwise $\mathbf{a}_{\infty}(t)$ is a Misiurewicz parameter, $R_{\mathbf{a}_{\infty}(t)}^{\infty}\left(\frac{t}{d}+\frac{l}{d}\right)$ and $R_{\mathbf{a}_{\infty}(t)}^{\infty}\left(\frac{t}{d}+\frac{l+1}{d}\right)$ land at $-\mathbf{a}_{\infty}(t)$.

Proof. - We will use the notations: $t_{0}=\frac{t}{d}+\frac{\left\lfloor\frac{d-1}{2}\right\rfloor}{d}, t_{1}=\frac{t}{d}+\frac{\left\lfloor\frac{d+1}{2}\right\rfloor}{d}$ and $x_{0}, x_{1}$ for the landing points of $R_{\mathbf{a}_{\infty}}^{\infty}\left(t_{0}\right), R_{\mathbf{a}_{\infty}}^{\infty}\left(t_{1}\right)$ respectively. We distinguish two cases according to whether $t_{0}, t_{1}$ are periodic or not.

(1) $t_{0}$ is periodic: The point $x_{0}$, which is eventually critical or parabolic, is now periodic and in the Julia set. So it is necessarily parabolic. If $t_{0}$ is fixed the result follows. Assume now that $t_{0}$ is not fixed. Suppose, to get a contradiction, that $x_{0}$ is not the root of $P_{t}$. So some ray with angle $t_{2}=d^{i} t_{0} \neq t_{0}$ converges to the root of the Fatou component $P_{t}$ which contains the critical point, that is to the image $f_{\mathbf{a}}^{i}\left(x_{0}\right)$ belonging to $\partial P_{t}$. Since the angles $\left\{\frac{k}{d^{j}(d-1)}, k \in \mathbf{N}, j \in \mathbf{N}\right\}$ are dense in $\mathbf{R} / \mathbf{Z}$ (the distance between two consecutive terms tends to 0 ), there exist three angles of this form called $\theta_{j} \neq 0$ separating $t_{0}, t_{1}$ and $t_{2}$. Hence the external rays of angles $\theta_{j}$ with the internal rays which have the same end points form a graph $\delta$ that separates $\mathbf{C}$ into three connected components: one contains $R_{\mathbf{a}_{\infty}(t)}^{\infty}\left(t_{2}\right)$ and the critical point, the other ones contain $R_{\mathbf{a}_{\infty}(t)}^{\infty}\left(t_{0}\right)$ and $R_{\mathbf{a}_{\infty}(t)}^{\infty}\left(t_{1}\right)$. By Lemma $1.4, \delta$ varies continuously for $\mathbf{a} \in \mathcal{R}_{\infty}(t)$ since the critical point cannot be on $\gamma$ (Lemma 2.29). Here we get a contradiction since the critical point varies continuously, cannot cross $\delta$ and has to break the rays $R_{\mathbf{a}_{\infty}(t)}^{\infty}\left(t_{0}\right)$ and $R_{\mathbf{a}_{\infty}(t)}^{\infty}\left(t_{1}\right)$ (Lemma 2.29). Hence $x_{0}$ is the root of $P_{t}$. Let $x^{\prime}$ be the other preimage on $\partial P_{t}$ of $f_{\mathbf{a}_{\infty}(t)}\left(x_{0}\right)$. We want to prove now that $R_{\mathbf{a}_{\infty}(t)}^{\infty}\left(t_{1}\right)$ converges to $x^{\prime}$. The curve $\gamma$ formed by all the fixed rays 
(see Lemma 2.29) varies continuously with a on $\mathcal{R}_{\infty}(t)$ up to $\mathbf{a}_{\infty}(t)$ since at this parameter the critical point is not on $\gamma$. So the rays $R_{\mathbf{a}_{\infty}(t)}^{\infty}\left(t_{0}\right)$ and $R_{\mathbf{a}_{\infty}(t)}^{\infty}\left(t_{1}\right)$ are the only preimages of $R_{\mathbf{a}_{\infty}(t)}^{\infty}\left(d t_{0}\right)$ in the same connected component of $\mathbf{C} \backslash \gamma$ as $P_{t}$. So $R_{\mathbf{a}_{\infty}(t)}^{\infty}\left(t_{1}\right)$ converges to $x^{\prime}$.

If $t_{1}$ is periodic instead of $t_{0}$ the proof is the same.

(2) Neither $t_{0}$ nor $t_{1}$ is periodic: Assume for contradiction that $x_{0}$ is eventually parabolic, by the same argument using $\gamma$ as before, $R_{\mathbf{a}_{\infty}(t)}^{\infty}\left(t_{0}\right)$ lands at the root $p$ of $P_{t}$. But $t_{0}$ is not periodic, so there is another ray $R_{\mathbf{a}_{\infty}(t)}^{\infty}\left(d^{i} t_{0}\right)$ landing at $p$. Let $k$ be the first integer such that $d^{i+k} t_{0} \equiv d^{k} t_{0} \bmod 1$; then $f_{\mathbf{a}}^{k-1}(p)$ must be critical since two different rays land at this point: $R_{\mathbf{a}_{\infty}(t)}^{\infty}\left(d^{i+k-1} t_{0}\right)$ and $R_{\mathbf{a}_{\infty}(t)}^{\infty}\left(d^{k-1} t_{0}\right)$ which have the same image $R_{\mathbf{a}_{\infty}(t)}^{\infty}\left(d^{k} t_{0}\right)$. This gives a contradiction. Finally, by Lemma $1.4, x_{0}$ is eventually critical.

Proposition 2.32. - For $t \in \mathbf{Q}$, let $\mathbf{a}_{0}(t)$ be the landing point of $\mathcal{R}_{0}(t)$. We have:

1. if $\frac{t}{d-1}+\frac{\left\lfloor\frac{d-1}{2}\right\rfloor}{d-1}$ is periodic by multiplication by $d-1$, then $R_{\mathbf{a}_{0}(t)}^{0}\left(\frac{t}{d-1}+\frac{\left\lfloor\frac{d-1}{2}\right\rfloor}{d-1}\right)$ lands at a parabolic point which is the root of the Fatou component $P_{t}$ which contains $-\mathbf{a}_{0}(t)$;

2. otherwise, $R_{\mathbf{a}_{0}(t)}^{0}\left(\frac{t}{d-1}+\frac{\left\lfloor\frac{d-1}{2}\right\rfloor}{d-1}\right)$ lands at the critical point $-\mathbf{a}_{0}(t)$.

Proof. - The proof goes exactly as the one of Proposition 2.31 .

LEMMA 2.33. - Let a be a Misiurewicz point on $\partial \mathcal{H}_{j}$ with $j \in\{0, \infty\}$,

$$
R_{\mathbf{a}}^{r(\mathbf{a})}(t) \text { lands at } f_{\mathbf{a}}(-\mathbf{a}) \quad \Longleftrightarrow \quad \mathcal{R}_{j}(t) \text { lands at } \mathbf{a} \text {. }
$$

Proof. - The proof of the implication $\Rightarrow$ is exactly the same as in degree 2 (see [5]). The proof of $\Leftarrow$ is just the case 2 of Propositions 2.31 and 2.32 since - a cannot be at the same time eventually periodic and attracted by a parabolic point.

We can now give another proof of the following corollary:

COROLlaRY 2.27. - If $d$ is odd, $\mathbf{R}^{+}=\mathcal{R}_{\infty}^{\mathrm{Id}}(0) \cup \mathcal{R}_{0}^{\mathrm{Id}}(0) \cup\{*\}$ where $*$ is a Misiurewicz point.

Proof. - If $d=2 l+1, \mathbf{R}^{+} \cap \mathcal{H}_{0}=\mathcal{R}_{0}(0)$ so $t=0$ and $\frac{t}{d-1}+\frac{\left\lfloor\frac{d-1}{2}\right\rfloor}{d-1}=\frac{l}{d-1}=\frac{1}{2}$; this angle is not periodic by multiplication by $d-1$, neither the angle $\frac{l+1}{d-1}$ since their images are $l$ and $l+1$. Thus $\mathcal{R}_{0}(0)$ converges to a parameter $\mathbf{a}_{0}$ such that $R_{\mathbf{a}_{0}}^{0}\left(\frac{1}{2}\right)$ lands $-\mathbf{a}_{0}$. So $\mathbf{a}_{0}$ is a Misiurewicz point. The unique positive fixed point is $f_{\mathbf{a}_{0}}\left(-\mathbf{a}_{0}\right)$ so the fixed ray $R_{\mathbf{a}_{0}}^{\infty}(0) \subset \mathbf{R}^{+}$converges to $f_{\mathbf{a}_{0}}\left(-\mathbf{a}_{0}\right)$ and by Lemma $2.33, \mathcal{R}_{\infty}(0)$ lands at $\mathbf{a}_{0}$.

\section{Graphs, puzzles, para-graphs and para-puzzles}

This section is devoted to the construction of the puzzles and the para-puzzles (see on the subject [3], [9], [13]). We recall in Section 3.1 the graphs used in [17] and we construct in Section 3.2 the analogous graphs in the parameter plane, then we establish the relations between graphs and para-graphs (Sections 3.3 and 3.4) and show how to use them for the question of local connectivity (Section 3.5).

\subsection{Dynamical puzzles and graphs}

We define the puzzles and the graphs (as in [17]) when the Julia set is connected, i.e. for parameters in $\mathcal{C} \backslash \mathbf{R}^{-}$, and obtain the puzzles in the other cases through a holomorphic motion of these graphs. 
Fix $\mathbf{a} \in \mathcal{C} \backslash \mathbf{R}^{-}$. For large $l$ and $\theta_{ \pm}= \pm \frac{1}{(d-1)^{l}-1}$, the ray $R_{\mathbf{a}}^{0}\left(\theta_{ \pm}\right)$is well defined and converges to a point $x_{ \pm}$which is repelling ( $f_{\mathbf{a}}$ possesses at most one parabolic orbit). Let $\eta_{ \pm}$ be the angle of some external ray $R_{\mathbf{a}}^{\infty}\left(\eta_{ \pm}\right)$landing at $x_{ \pm}$(given by Proposition 1.5). Since the internal ray $R_{\mathbf{a}}^{0}\left(\theta_{ \pm}\right)$is fixed by $f_{\mathbf{a}}^{l}$, the external ray $R_{\mathbf{a}}^{\infty}\left(\eta_{ \pm}\right)$will also be fixed by $f_{\mathbf{a}}^{l}$ so $\eta_{ \pm}$is periodic (see Remark 3.2). Using these rays we construct the graphs in $\bar{X}^{\mathbf{a}}$, where $X^{\mathbf{a}}=\left\{z \in \mathbf{C} \mid G_{\mathbf{a}}^{0}(z)<1\right.$ and $\left.G_{\mathbf{a}}^{\infty}(z)<1\right\}$, as follows.

Definition 3.1. - Let $\mathbf{a} \in \mathcal{C} \backslash \mathbf{R}^{-}, \theta_{ \pm}= \pm \frac{1}{(d-1)^{l}-1}$ with $l$ large (as before). A puzzle for $f_{\mathbf{a}}$ is defined by the following graph:

$$
I_{0}^{\mathbf{a}}\left(\theta_{ \pm}\right)=\partial X^{\mathbf{a}} \cup\left(X^{\mathbf{a}} \cap\left(\bigcup_{i \geq 0}\left(\overline{R_{\mathbf{a}}^{0}\left((d-1)^{i} \theta_{ \pm}\right)} \cup \overline{R_{\mathbf{a}}^{\infty}\left(d^{i} \eta_{ \pm}\right)}\right)\right)\right) .
$$

The puzzle pieces of depth $n \geq 0$ are the connected components of

$$
f_{\mathbf{a}}^{-n}\left(X^{\mathbf{a}}\right) \backslash I_{n}^{\mathbf{a}}=f_{\mathbf{a}}^{-n}\left(X^{\mathbf{a}} \backslash I_{0}^{\mathbf{a}}\right), \quad \text { where } I_{n}^{\mathbf{a}}=f_{\mathbf{a}}^{-n}\left(I_{0}^{\mathbf{a}}\right) \text { for all } n \geqslant 1 .
$$

The puzzle piece containing a given point $z$ is denoted by $P_{n}^{\mathbf{a}}(z)$. The puzzle pieces containing the critical value $f_{\mathbf{a}}(-\mathbf{a})$ are denoted simply by $P_{0}^{\mathbf{a}}, \ldots, P_{n}^{\mathbf{a}}, \ldots$ if there is no ambiguity.

The puzzle is the union of all the puzzle pieces.

Remark 3.2. - The ray $R_{\mathbf{a}}^{\infty}\left(\eta_{ \pm}\right)$is the only external ray of the cycle $R_{\mathbf{a}}^{\infty}\left(d^{j} \eta_{ \pm}\right), j \geqslant 0$, to converge to $x_{ \pm}$.

Proof. - Assume (to get a contradiction) that $R_{\mathbf{a}}^{\infty}\left(d^{j} \eta_{ \pm}\right)$with $d^{j} \eta_{ \pm} \neq \eta_{ \pm} \bmod 1$ converges to $x_{ \pm}$. Since there is a finite number of rays in the cycle converging to $x_{ \pm}$we can assume (up to changing the notations) that the angles are all in the interval $\left(\eta_{ \pm}, d^{j} \eta_{ \pm}\right)$. Since the map $f_{\mathbf{a}}^{l-j}$ is conformal at this point, it preserves the "cyclic order" of the rays at $x_{ \pm}$. But it maps $R_{\mathbf{a}}^{\infty}\left(d^{j} \eta_{ \pm}\right)$ to $R_{\mathbf{a}}^{\infty}\left(\eta_{ \pm}\right)$and $R_{\mathbf{a}}^{\infty}\left(\eta_{ \pm}\right)$to $R_{\mathbf{a}}^{\infty}\left(d^{l-j} \eta_{ \pm}\right)$. These rays land at $x_{ \pm}$but (because of the cyclic order) $d^{l-j} \eta_{ \pm}$will not be in the interval $\left(\eta_{ \pm}, d^{j} \eta_{ \pm}\right)$. So the two rays cannot be in the same cycle.

If we fix some $\theta$ as in Definition 3.1 but vary the parameter $\mathbf{a}$ in $\mathbf{C} \backslash \mathbf{R}^{-}$, we will see that for the graph $I_{0}^{\mathbf{a}}(\theta)$ the landing points of the rays, $x_{ \pm}$, can become parabolic, the rays $R_{\infty}^{\mathbf{a}}\left(\eta_{ \pm}\right)$and $R_{\mathbf{a}}^{0}\left(\theta_{ \pm}\right)$can land at different points, the rays can crash on critical points and no more be welldefined etc. For these reasons we should restrict the domain (in the parameter space) on which we consider the graph at each depth. The para-puzzle pieces defined in Section 3.2 correspond exactly to the region where the dynamical pieces are defined by the same rays.

\subsection{Para-graphs and para-puzzles}

The para-graphs are just the copy of dynamical graphs in the parameter plane so depend from a preferred parameter. Let $\mathbf{a}_{0} \in \mathcal{C} \backslash \mathbf{R}^{-}$and $I_{0}^{\mathbf{a}_{0}}(\theta)$ be the graph associated to this parameter by Definition 3.1, with $\theta \in\left\{\theta_{ \pm}\right\}$.

Definition 3.3. - For $n \in \mathbf{N}$, let $\kappa_{n}$ be the set of all the pairs $(\mathcal{U}, v)$ where:

- $\mathcal{U}$ is a connected component of $\mathcal{H}_{i}$ with $i \in\{\infty, 0, \ldots, n\}$;

$-(d-1)^{n-i} v=1$ if $0 \leqslant i \leqslant n$ and $d^{n} v=1$ if $i=\infty$.

Let $\mathcal{X}_{n}$ be the connected component containing $\mathbf{a}_{0}$ of $\mathbf{C} \backslash\left(\bigcup_{(\mathcal{U}, v) \in \kappa_{n}} \overline{\mathcal{E}_{\mathcal{U}}(v)}\right)$.

Definition 3.4. - For $n \in \mathbf{N}$, let $\Theta_{n}$ be the set of all the pairs $(\mathcal{U}, t)$ where:

- $\mathcal{U}$ is a connected component of $\mathcal{H}_{i}$ with $i \in\{\infty, 0, \ldots, n\}$;

$-(d-1)^{n-i} t \in\left\{\theta, \ldots,(d-1)^{l-1} \theta\right\}$ if $0 \leqslant i \leqslant n$;

- $d^{n} t \in\left\{d^{j} \eta, j \geqslant 0\right\}$ if $i=\infty$.

$4^{\text {e }}$ SÉRIE - TOME $40-2007-\mathrm{N}^{\circ} 6$ 

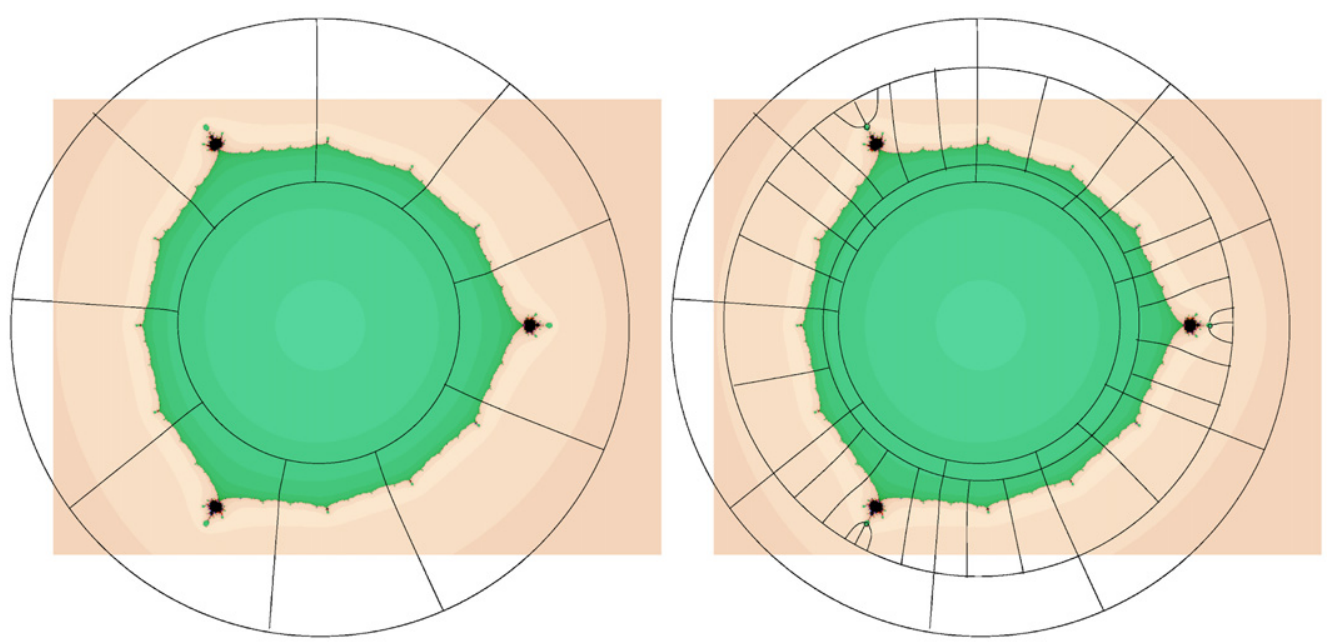

Fig. 7. Schematic representation of para-graphs $\mathcal{I}_{0}$ and $\mathcal{I}_{1}$ for $\mathcal{C}_{4}$.

Definition 3.5. - The para-puzzle is the union of the para-puzzle pieces. The para-puzzle pieces of depth $n$ are the connected components of $\mathcal{X}_{n} \backslash \mathcal{I}_{n}$ where

$$
\mathcal{I}_{n}(\theta)=\partial \mathcal{X}_{n} \cup \bigcup_{(\mathcal{U}, t) \in \Theta_{n}}\left(\overline{\cup \mathcal{R}_{\mathcal{U}}(t)} \cap \mathcal{X}_{n}\right)
$$

The para-puzzle piece containing a given parameter a will be denoted by $\mathcal{P}_{n}(\mathbf{a})$. For the given parameter $\mathbf{a}_{0}$ we will simply write $\mathcal{P}_{n}$ for $\mathcal{P}_{n}\left(\mathbf{a}_{0}\right)$.

The points of $\mathbf{R}^{-}$can be added or not to the graph. We only care of parameters in $\mathcal{S}$ and for these parameters, all the para-puzzle pieces are compactly contained in $\mathbf{C} \backslash \mathbf{R}^{-}$by the following remark.

Remark 3.6. - For parameters $\mathbf{a}_{0} \in \mathcal{S}$, the para-puzzle piece $\mathcal{P}_{0}\left(\mathbf{a}_{0}\right)$ is compactly contained in $\mathbf{C} \backslash \mathbf{R}^{-}$. Moreover there is only one connected component of $\cup \mathcal{R}_{p}(t) \backslash\{p\}$ in $\mathcal{P}_{0}\left(\mathbf{a}_{0}\right)$. Thus for the simplicity of the exposition we will forget the exponent in $\mathcal{R}_{p}^{s}(t)$ and call $\mathcal{R}_{p}(t)$ the parameter ray that belongs to $\mathcal{P}_{0}\left(\mathbf{a}_{0}\right)$.

Proof. - By definition of the para-graphs, any piece of depth 0 is bounded only by rays in $\mathcal{H}_{0}$ and in $\mathcal{H}_{\infty}$ and equipotentials. If $\mathcal{P}_{0}\left(\mathbf{a}_{0}\right)$ intersects $\mathbf{R}^{+}$then it is compactly contained in the interior of $\mathcal{S} \cup \sigma \mathcal{S} \cup \tau^{-1} \mathcal{S}$. Indeed, the sector $\sigma \mathcal{S} \cup \tau^{-1} \mathcal{S}=\rho^{-1}(\mathcal{S} \cup \sigma \mathcal{S})$ with $\rho=e^{\frac{i \pi}{d-1}}$ contains all the rays of $\mathcal{H}_{0}$ in a sector of angles of width greater than $1+\frac{1}{d-2}$ by proposition 2.4 and using the coordinate $\Phi_{0}$. Therefore, $\mathcal{P}_{0}\left(\mathbf{a}_{0}\right)$ is contained in the interior of $\mathcal{S} \cup \sigma \mathcal{S} \cup \tau^{-1} \mathcal{S}$ since by definition $\mathcal{P}_{0}\left(\mathbf{a}_{0}\right) \cap \mathcal{H}_{0}$ corresponds to angles in a sector of width less than 1 . Using the same argument, if $\mathcal{P}_{0}\left(\mathbf{a}_{0}\right)$ intersects $\rho \mathbf{R}^{+}$then it is compactly contained in the interior of $\mathcal{S} \cup \tau \sigma \mathcal{S} \cup \tau \mathcal{S}$ which is $\mathcal{S} \cup \tau(\mathcal{S} \cup \sigma \mathcal{S})$. Hence, $\mathcal{P}_{0}\left(\mathbf{a}_{0}\right)$ is compactly contained in $\mathbf{C} \backslash \mathbf{R}^{-}$since the interior of $\mathcal{S} \cup \rho \mathcal{S} \cup \sigma \mathcal{S}$ is included in $\mathbf{C} \backslash \mathbf{R}^{-}$.

Since by definition $\mathcal{P}_{0}\left(\mathbf{a}_{0}\right) \cap \mathcal{H}_{0}$ corresponds to angles in a sector of width less than 1 , $\cup \mathcal{R}_{0}(t) \backslash\{0\}$ has only one connected component in $\mathcal{P}_{0}\left(\mathbf{a}_{0}\right)$. The same holds for $\cup \mathcal{R}_{\infty}(t) \backslash$ $\{\infty\}$. 


\subsection{Holomorphic motion of the dynamical graphs}

Definition 3.7. - Let $\Lambda$ be a $\mathbf{C}$-analytic variety. Let $\lambda_{0} \in \Lambda$. A holomorphic motion of a subset $\Gamma \subset \overline{\mathbf{C}}$ parameterized by $\Lambda$ is a map $\Psi: \Lambda \times \Gamma \rightarrow \overline{\mathbf{C}}$ such that $\Psi(., z)$ is holomorphic on $\Lambda, \Psi^{\lambda}=\Psi(\lambda,$.$) is injective on \Gamma$ and $\Psi^{\lambda_{0}}=\mathrm{Id}$.

For the given parameter $\mathbf{a}_{0} \in \mathcal{C} \backslash \mathbf{R}^{-}$, we define now the set of parameters for which the graph $I_{0}^{\mathbf{a}_{0}}(\theta)$ admits a holomorphic motion. Let $\eta$ be the angle of the external ray converging to the same point as $R_{\mathbf{a}_{0}}^{0}(\theta)$ in $I_{0}^{\mathbf{a}_{0}}(\theta)$.

LEMMA 3.8. - Let $\Omega_{\eta}$ be the set of parameters $\mathbf{a} \in \mathbf{C} \backslash \mathbf{R}^{-}$such that for all $i \geqslant 0$ the ray $R_{\mathbf{a}}^{\infty}\left(d^{i} \eta\right)$ is well-defined and converges to a repelling periodic point.

1. $\Omega_{\eta}$ is a non-empty open set;

2. the set $\Gamma_{\mathbf{a}_{0}}^{\infty}(\eta)=\bigcup_{i \geqslant 0} \overline{R_{\mathbf{a}_{0}}^{\infty}\left(d^{i} \eta\right)}$ admits a holomorphic motion parameterized by $\Omega_{\eta}$;

3. the boundary $\partial \Omega_{\eta}$ is a subset of $\mathbf{R}^{-} \cup \bigcup_{i \geqslant 0} \overline{\cup \mathcal{R}_{\infty}\left(d^{i} \eta\right)}$ without isolated points.

Proof. -1 and 2: Since $\mathbf{a}_{0} \in \Omega_{\eta}$, it is clearly not empty. To prove that $\Omega_{\eta}$ is open, take $\mathbf{a}_{1} \in \Omega_{\eta}$. For each $i \geqslant 0$, the landing point of $R_{\mathbf{a}_{1}}^{\infty}\left(d^{i} \eta\right)$ is periodic and repelling, thus not eventually critical. Therefore the compact set $\overline{R_{\mathbf{a}_{1}}^{\infty}\left(d^{i} \eta\right)}$ admits a holomorphic motion in some neighbourhood of $\mathbf{a}_{1}$ (Lemma 1.4). The set $\Gamma_{\mathbf{a}_{1}}^{\infty}(\eta)$ is a finite union of such compact sets, so it admits a holomorphic motion in a neighbourhood of $\mathbf{a}_{1}$. Hence $\Omega_{\eta}$ is open.

3. a) Remark that $\mathbf{R}^{-} \subset \mathbf{C} \backslash \Omega_{\eta}$ (by definition), so there is no isolated point of $\partial \Omega_{\eta}$ in $\mathbf{R}^{-}$. Assume for contradiction that there is a point $\mathbf{a}_{1}$ of $\mathbf{C} \backslash \mathbf{R}^{-}$isolated in $\partial \Omega_{\eta}=\overline{\Omega_{\eta}} \backslash \Omega_{\eta}$. Then there exists an open neighbourhood $\mathcal{O}$ of $\mathbf{a}_{1}$ such that $\mathcal{O} \backslash\left\{\mathbf{a}_{1}\right\} \subset \Omega_{\eta}$. Since the parameter $\mathbf{a}_{1}$ is not in $\Omega_{\eta}$, one of the rays either is not well defined or converges to a parabolic point. If the ray $R_{\mathbf{a}_{1}}^{\infty}\left(d^{i} \eta\right)$ crashes on $-\mathbf{a}_{1}$, then $f_{\mathbf{a}_{1}}\left(-\mathbf{a}_{1}\right)$ belongs to $R_{\mathbf{a}_{1}}^{\infty}\left(d^{i+1} \eta\right)$ and $\mathbf{a}_{1} \in \mathcal{R}_{\infty}^{s}\left(d^{i+1} \eta\right)$ for some $s$ composition of $\tau$ and $\sigma$. Then for parameters $\mathbf{a} \in \mathcal{R}_{\infty}^{s}\left(d^{i+1} \eta\right) \cap \mathcal{O}$ near $\mathbf{a}_{1}$, the ray $R_{\mathbf{a}}^{\infty}\left(d^{i} \eta\right)$ also crashes. This contradicts the fact that $\mathcal{O} \backslash\left\{\mathbf{a}_{1}\right\} \subset \Omega_{\eta}$. Consider now the case where all the rays $R_{\mathbf{a}_{1}}^{\infty}\left(d^{i} \eta\right)$ are well-defined but converge to a parabolic periodic cycle ( $d^{i} \eta$ is periodic). For $\mathbf{a} \in \mathcal{O}$, the landing point $x_{i}(\mathbf{a})$ of the ray $R_{\mathbf{a}}^{\infty}\left(d^{i} \eta\right)$ defines a holomorphic map (Lemma 1.4). It is repelling for $\mathbf{a} \in \mathcal{O} \backslash\left\{\mathbf{a}_{1}\right\}$ and parabolic at $\mathbf{a}_{1}$. So $\left(f_{\mathbf{a}}^{k}\right)^{\prime}(x(\mathbf{a}))$ can be extended to a holomorphic map from $\mathcal{O}$ to $\mathbf{C} \backslash \mathbf{D}$ (where $k$ denotes the period of the cycle). Its modulus reaches its minimum at $\mathbf{a}_{1}$; this contradicts the maximum principle for the map $\mathbf{a} \mapsto 1 /\left(f_{\mathbf{a}}^{k}\right)^{\prime}(x(\mathbf{a}))$.

b) For $\mathbf{a} \in \partial \Omega_{\eta} \backslash \mathbf{R}^{-}$, either one ray $R_{\mathbf{a}}^{\infty}\left(d^{i} \eta\right)$ crashes on the critical point $-\mathbf{a}$ and so $\mathbf{a} \in \cup \mathcal{R}_{\infty}\left(d^{i+1} \eta\right)$, or the rays $R_{\mathbf{a}}^{\infty}\left(d^{i} \eta\right)$ converge to a parabolic periodic cycle and so $\mathbf{a} \in \mathcal{P} \mathcal{A}=$ $\left\{\mathbf{a} \mid \exists x\right.$ such that $f_{\mathbf{a}}^{k}(x)=x$ and $\left.\left(f_{\mathbf{a}}^{k}\right)^{\prime}(x)=1\right\}$ (where $k$ is the period of $\eta$ ). The set $\mathcal{P} \mathcal{A}$ is finite since every $\mathbf{a} \in \mathcal{P} \mathcal{A}$ is a root of the discriminant of the two polynomials $f_{\mathbf{a}}^{k}(z)-z$ and $\left(f_{\mathbf{a}}^{k}\right)^{\prime}(z)-1$. Since there is no isolated points in $\partial \Omega_{\eta}$ those parameters of $\mathcal{P} \mathcal{A} \cap \partial \Omega_{\eta}$ are in the closure $\cup \mathcal{R}_{\infty}\left(d^{i} \eta\right)$ for $i \geqslant 0$.

The same result holds for internal rays:

LEMMA 3.9. - Let $\Omega_{\theta}^{\prime}$ be the set of parameters $\mathbf{a} \in \mathbf{C} \backslash \mathbf{R}^{-}$such that for all $i \geqslant 0$ the ray $R_{\mathbf{a}}^{0}\left((d-1)^{i} \theta\right)$ is well-defined and converges to a repelling periodic point.

1. $\Omega_{\theta}^{\prime}$ is a non-empty open set;

2. the set $\Gamma_{\mathbf{a}_{0}}^{0}(\theta)=\bigcup_{i \geqslant 0} \overline{R_{\mathbf{a}_{0}}^{0}\left((d-1)^{i} \theta\right)}$ admits a holomorphic motion parameterized by $\Omega_{\theta}^{\prime}$;

3. the boundary $\partial \Omega_{\theta}^{\prime}$ is a subset of $\mathbf{R}^{-} \cup \bigcup_{i \geqslant 0} \overline{\cup \mathcal{R}_{0}\left((d-1)^{i} \theta\right)}$. It has no isolated points.

COROLLARY 3.10. - In the connected component containing $\mathbf{a}_{0}$ of $\Omega_{\eta} \cap \Omega_{\theta}^{\prime}$, the rays $R_{\mathbf{a}}^{0}(\theta)$ and $R_{\mathbf{a}}^{\infty}(\eta)$ land at a common point.

$4^{\text {e }}$ SÉRIE - TOME $40-2007-\mathrm{N}^{\circ} 6$ 
Proof. - The landing points $x_{0}(\mathbf{a})$ of $R_{\mathbf{a}}^{0}(\theta), x_{\infty}(\mathbf{a})$ of $R_{\mathbf{a}}^{\infty}(\eta)$, are both repelling periodic points. The period is determined by the angles $\theta$, and $\eta$, and is at most say $k$. At the parameter $\mathbf{a}_{0}$ the points coincide by definition of the graph: $x=x_{0}\left(\mathbf{a}_{0}\right)=x_{\infty}\left(\mathbf{a}_{0}\right)$. Since $x$ is repelling, by Rouché's Theorem, on some neighbourhood $U$ of $x$ there is exactly one point of period less than $k$, for $\mathbf{a}$ in a neighbourhood $\mathcal{U} \subset \Omega_{\eta} \cap \Omega_{\theta}^{\prime}$ of $\mathbf{a}_{0}$. Moreover, the points $x_{0}(\mathbf{a})$ and $x_{\infty}(\mathbf{a})$ vary continuously for $\mathbf{a} \in \Omega_{\eta} \cap \Omega_{\theta}^{\prime}$ (Lemmas 3.8 and 3.9). Therefore, they coincide on $\mathcal{U}$ and finally on the connected component containing $\mathbf{a}_{0}$ of $\Omega_{\eta} \cap \Omega_{\theta}^{\prime}$ (since they are holomorphic maps).

COROLLARY 3.11. - The para-puzzle piece $\mathcal{P}_{0}$ is contained in the connected component of $\Omega_{\eta} \cap \Omega_{\theta}^{\prime}$ containing $\mathbf{a}_{0}$. Therefore the graph $I_{0}^{\mathbf{a}_{0}}(\theta)$ admits a holomorphic motion defined on $\mathcal{P}_{0}\left(\mathbf{a}_{0}\right)$ so that $I_{0}^{\mathbf{a}}(\theta)$ is well defined (i.e. the rays of the graph are well defined).

Proof. - The boundary $\partial \Omega_{\theta}^{\prime}$ is included in $\Omega_{\eta} \cup \mathbf{R}^{-}$, except for the landing points of the rays, since $\partial \Omega_{\theta}^{\prime} \cap \partial \Omega_{\eta} \subset \mathbf{R}^{-} \cup\left(\overline{\cup \mathcal{R}_{0}\left((d-1)^{i} \theta\right)} \cap \overline{\cup \mathcal{R}_{\infty}\left(d^{i} \eta\right)}\right)$. The same holds for $\partial \Omega_{\eta}$, so the boundary of $\Omega_{\eta} \cap \Omega_{\theta}^{\prime}$ is simply the union $\partial \Omega_{\eta} \cup \partial \Omega_{\theta}^{\prime}$. Thus $\left(\partial \Omega_{\eta} \cup \partial \Omega_{\theta}^{\prime}\right) \cap \mathcal{X}_{0}$ is included in $\mathcal{I}_{0}(\theta) \cup \mathbf{R}^{-}$. Therefore $\mathcal{P}_{0}$ is contained in the connected component of $\Omega_{\eta} \cap \Omega_{\theta}^{\prime}$ containing $\mathbf{a}_{0}$ (since $\mathcal{P}_{0}=\mathcal{P}_{0}\left(\mathbf{a}_{0}\right)$ is a connected component of $\mathbf{C} \backslash \mathcal{I}_{0}(\theta)$ in $\mathbf{C} \backslash \mathbf{R}^{-}$).

Hence, the graph $I_{0}^{\mathbf{a}}(\theta)=\partial X_{\mathbf{a}} \cup\left(\left(\Gamma_{\mathbf{a}}^{\infty}(\eta) \cup \Gamma_{\mathbf{a}}^{0}(\theta)\right) \cap X_{\mathbf{a}}\right)$ is well defined for a in $\mathcal{P}_{0}\left(\mathbf{a}_{0}\right)$, since $\mathcal{P}_{0}\left(\mathbf{a}_{0}\right)$ is included in $\mathcal{X}_{0}$. The holomorphic motion of the graph $I_{0}^{\mathbf{a}_{0}}$ follows from Lemma 3.8, Lemma 3.9 and the fact that the map $(\mathbf{a}, z) \rightarrow \phi_{\mathbf{a}}^{p} \circ\left(\phi_{\mathbf{a}_{0}}^{p}\right)^{-1}(z)$ defines a holomorphic motion of the equipotentials $E_{\mathbf{a}_{0}}^{p}(1)$ for $\mathbf{a}$ in $\mathcal{X}_{0}$ and $p \in\{0, \infty\}$.

From now on through the rest of the paper, we restrict ourself to parameters $\mathbf{a}_{0} \in \mathcal{S}$ and study para-puzzles inside the open region $\mathcal{P}_{0}\left(\mathbf{a}_{0}\right)$. Hence by Remark 3.6 we don't need assumptions on the sector containing the parameters considered.

COROLLARY 3.12. - For $n \geqslant 1$, the points which are in $\mathcal{P}_{0}$ of $\mathcal{I}_{n}(\theta) \cap \mathcal{C}$ are of Misiurewicz type.

Proof. - A parameter $\mathbf{a} \in \mathcal{I}_{n}(\theta) \cap \partial \mathcal{C}$ is necessarily the landing point of a ray $\mathcal{R}_{\infty}(t)$ with $d^{n} t \in\left\{d^{j} \eta, j \geqslant 0\right\}$ (by definition of $\mathcal{I}_{n}(\theta)$ ). Thus the ray $R_{\mathbf{a}}^{\infty}(t)$ belongs to $I_{n}^{\mathbf{a}}$ since its image by $f_{\mathbf{a}}^{n}, R_{\mathbf{a}}^{\infty}\left(d^{n} t\right)$, belongs to $I_{0}^{\mathbf{a}}$ (by definition of $t$ and of $I_{0}^{\mathbf{a}}$ ). Since they are in $\mathcal{P}_{0}$, the landing points of rays in $I_{0}^{\mathrm{a}}$ are repelling periodic points (Lemma 3.8 and Corollary 3.10). Therefore a is a Misiurewicz point since we are in the second alternative of Lemma 2.24: $R_{\mathbf{a}}^{\infty}\left(d^{n} t\right)$ lands at a repelling periodic point.

LEMMA 3.13. - For parameters $\mathbf{a} \in \mathcal{P}_{0}\left(\mathbf{a}_{0}\right)$, the following equivalence holds:

$$
\mathbf{a} \in \mathcal{I}_{n} \quad \Leftrightarrow \quad f_{\mathbf{a}}(-\mathbf{a}) \in I_{n}^{\mathbf{a}} .
$$

Proof. - By construction of $\mathcal{I}_{n}$, the rays and equipotentials involved in $\mathcal{I}_{n}$ and $I_{n}^{\text {a correspond to }}$ each other via the change of coordinates (Remark 2.16 and Corollary 2.23). From Corollary 3.12 and its proof, the points in $\mathcal{I}_{n} \cap \partial \mathcal{C}$ are Misiurewicz points and $f_{\mathbf{a}}(-\mathbf{a})$ is the landing point of the corresponding ray in $I_{n}^{\mathbf{a}}$. Conversely, if $f_{\mathbf{a}}(-\mathbf{a}) \in I_{n}^{\mathbf{a}}$ is in the Julia set, it is the landing point of some external ray $R_{\mathbf{a}}^{\infty}(t)$ of $I_{n}^{\mathbf{a}}$, so $d^{n} t \in\left\{d^{j} \eta, j \geqslant 0\right\}$. Since $f_{\mathbf{a}}(-\mathbf{a})$ is eventually periodic, a is a Misiurewicz point and by Lemma 2.26 the external ray $\mathcal{R}_{\infty}(t)$ lands at a. Hence, the parameter a belongs to $\mathcal{I}_{n}$ (by definition of this para-graph).

COROLlaRY 3.14. - For $\mathbf{a} \in \mathcal{P}_{n}\left(\mathbf{a}_{0}\right)$, the n-th para-puzzle piece, the critical point $-\mathbf{a}$ is not on any of the graphs $I_{0}^{\mathrm{a}}, \ldots, I_{n+1}^{\mathrm{a}}$.

COROLLARY 3.15. - The para-puzzle pieces are simply connected. 
Proof. - It is equivalent to prove that the graph $\mathcal{I}_{n}$ is connected. Any part of an equipotential involved in $\mathcal{I}_{n} \cap \mathcal{H}_{i}(i \in \mathbf{N})$ is connected to $\partial \mathcal{C}$ by a ray in $\mathcal{I}_{n}$. By Corollary 3.12 , this ray converges to a Misiurewicz parameter, say $\mathbf{a}_{1}$. At this parameter, in the dynamical graph $I_{n}^{\mathbf{a}_{1}}$, some external ray $R_{\mathbf{a}_{1}}^{\infty}\left(t^{\prime}\right)$ converges to $f_{\mathbf{a}_{1}}\left(-\mathbf{a}_{1}\right)$. Then the external parameter ray $\mathcal{R}_{\infty}\left(t^{\prime}\right)$ of $\mathcal{I}_{n}$ (by Lemma 3.13) converges to $\mathbf{a}_{1}$ (Lemma 2.26). Finally all these external rays are connected to the external equipotential of the graph $\mathcal{I}_{n}$.

Let $n \geqslant 1$ and $\mathcal{P}_{n}=\mathcal{P}_{n}\left(\mathbf{a}_{0}\right) \subset \mathcal{P}_{n-1}=\mathcal{P}_{n-1}\left(\mathbf{a}_{0}\right)$.

LEMMA 3.16. - There exists a holomorphic motion $h_{n}: \mathcal{P}_{n} \times I_{n+1}^{\mathbf{a}_{0}} \rightarrow \overline{\mathbf{C}}$ such that:

$-I_{n+1}^{\mathbf{a}}=h_{n}^{\mathbf{a}}\left(I_{n+1}^{\mathbf{a}_{0}}\right)$ for all $\mathbf{a} \in \mathcal{P}_{n}$;

- $h_{n}$ coincides with $h_{n-1}$ on $\mathcal{P}_{n} \times I_{n}^{\mathbf{a}_{0}}$;

- for every $\mathbf{a} \in \mathcal{P}_{n}$ the following diagram is commutative:

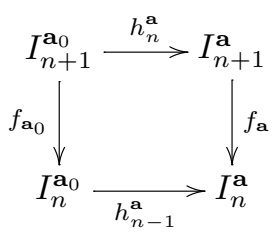

Proof. - By Corollary 3.11, the graph $I_{0}^{\mathbf{a}_{0}}$ admits a holomorphic motion on $\mathcal{P}_{0}\left(\mathbf{a}_{0}\right)$. For $\mathbf{a} \in \mathcal{P}_{n}$ the critical point is not on any of the graphs $I_{k}^{\mathrm{a}}$ for $k \leqslant n+1$ (Corollary 3.14), so we can pullback the holomorphic motion of $I_{0}^{\mathbf{a}_{0}}$ (by $f_{\mathbf{a}}^{j}$ with $j \leqslant n+1$ ) to get the sequence of holomorphic motions of the graphs $I_{j}^{\mathbf{a}_{0}}$ on the restricted domain $\mathcal{P}_{j-1}$. By construction they satisfy the announced properties.

\subsection{Relation between graphs and para-graphs}

LEMMA 3.17. - The following map $H_{n}$ is a homeomorphism.

$$
H_{n}:\left\{\begin{aligned}
\mathcal{P}_{n} \cap \mathcal{I}_{n+1} & \longrightarrow P_{n}^{\mathbf{a}_{0}} \cap I_{n+1}^{\mathbf{a}_{0}} \\
\mathbf{a} & \longmapsto\left(h_{n}^{\mathbf{a}}\right)^{-1}\left(f_{\mathbf{a}}(-\mathbf{a})\right) .
\end{aligned}\right.
$$

Proof. - For a in $\mathcal{P}_{n} \cap \mathcal{I}_{n+1},\left(h_{n}^{\mathbf{a}}\right)^{-1}\left(f_{\mathbf{a}}(-\mathbf{a})\right)$ is well-defined by Lemmas 3.16 and 3.13. The image $H_{n}\left(\mathcal{P}_{n} \cap \mathcal{I}_{n+1}\right)$ is clearly included in $I_{n+1}^{\mathbf{a}_{0}}$. Moreover for $\mathbf{a}=\mathbf{a}_{0}$, the critical value $f_{\mathbf{a}_{0}}\left(-\mathbf{a}_{0}\right)$ belongs to the puzzle piece $P_{n}^{\mathbf{a}_{0}}$. Therefore $f_{\mathbf{a}}(-\mathbf{a})$ belongs to the (open) puzzle piece bounded by $h_{n}^{\mathbf{a}}\left(\partial P_{n}^{\mathbf{a}_{0}}\right)$, since $f_{\mathbf{a}}(-\mathbf{a})$ and $I_{n}^{\mathbf{a}}=h_{n}^{\mathbf{a}}\left(I_{n}^{\mathbf{a}_{0}}\right)$ move continuously and never meet when $\mathbf{a} \in \mathcal{P}_{n}$ (Corollary 3.14). Hence, $H_{n}\left(\mathcal{P}_{n} \cap \mathcal{I}_{n+1}\right) \subset P_{n}^{\mathbf{a}_{0}}$ since $\left(h_{n}^{\mathbf{a}}\right)^{-1}$ is injective on $I_{n+1}^{\mathbf{a}}$.

By construction, the map $H_{n}$ is clearly a homeomorphism on the rays and equipotentials of $\mathcal{I}_{n+1} \cap \mathcal{P}_{n}$ that are in $\mathcal{H}_{\infty}$. We prove now that it is injective in $\mathcal{H}$. Assume by contradiction that $\mathcal{U}_{1}, \mathcal{U}_{2}$ are two connected components of $\mathcal{H}$ and that there exist parameters $\mathbf{a}_{1}, \mathbf{a}_{2}$ on two rays $\mathcal{R}_{\mathcal{U}_{1}}\left(t_{1}\right), \mathcal{R}_{\mathcal{U}_{2}}\left(t_{2}\right)$ respectively such that $H_{n}\left(\mathbf{a}_{1}\right)=H_{n}\left(\mathbf{a}_{2}\right)$. Since $\mathcal{P}_{n}$ is a simply connected region (Corollary 3.15) that avoids the center of all the components of $\mathcal{H}_{i}$ for $0 \leqslant i \leqslant n$, we can define functions $r_{\mathcal{U}_{1}}(\mathbf{a})$ and $r_{\mathcal{U}_{2}}(\mathbf{a})$ on $\mathcal{P}_{n}$ by Lemma 2.22. Since for $j=1,2$ the critical value $f_{\mathbf{a}_{j}}\left(-\mathbf{a}_{j}\right)$ belongs to $R_{\mathbf{a}_{j}}^{r_{\mathcal{U}_{j}}\left(\mathbf{a}_{j}\right)}\left(t_{j}\right)$ (Corollary 2.23), $H_{n}\left(\mathbf{a}_{j}\right)$ then belongs to $\left(h_{n}^{\mathbf{a}_{j}}\right)^{-1}\left(R_{\mathbf{a}_{j}}^{r_{\mathcal{U}_{j}}\left(\mathbf{a}_{j}\right)}\left(t_{j}\right)\right)=R_{\mathbf{a}_{0}}^{r_{\mathcal{U}_{j}}\left(\mathbf{a}_{0}\right)}\left(t_{j}\right)$. Since $H_{n}\left(\mathbf{a}_{1}\right)=H_{n}\left(\mathbf{a}_{2}\right)$, the two rays have a common point so coincide, and $\mathcal{U}_{1}=\mathcal{U}_{2}, t_{1}=t_{2}$. The same arguments work (simpler) for the injectivity on the equipotentials in $\mathcal{H} \cap \mathcal{I}_{n+1}$.

To achieve the proof of injectivity, it is enough to show that $H_{n}$ is injective on $\mathcal{I}_{n+1} \cap \partial \mathcal{C}$. Thus, we consider two distinct rays $\mathcal{R}_{\mathcal{U}_{1}}\left(t_{1}\right), \mathcal{R}_{\mathcal{U}_{2}}\left(t_{2}\right) \subset \mathcal{I}_{n+1}$ landing at points $\mathbf{a}_{1}, \mathbf{a}_{2} \in \mathcal{P}_{n}$ such that

$4^{e}$ SÉRIE - TOME $40-2007-\mathrm{N}^{\circ} 6$ 
$H_{n}\left(\mathbf{a}_{1}\right)=H_{n}\left(\mathbf{a}_{2}\right)$, with $\mathcal{U}_{j}$ connected components of $\mathcal{H} \cup \mathcal{H}_{\infty}$. As before, the corresponding dynamical rays $R_{\mathbf{a}_{j}}^{r \mathbf{U}_{j}\left(\mathbf{a}_{j}\right)}\left(t_{j}\right)$ for $j=1,2$ are pulled back by the holomorphic motion to the rays $R_{\mathbf{a}_{0}}^{r \mathcal{U}_{j}\left(\mathbf{a}_{0}\right)}\left(t_{j}\right)$. These rays land at a common point: $H_{n}\left(\mathbf{a}_{1}\right)=H_{n}\left(\mathbf{a}_{2}\right)$. Since this point is eventually repelling and not eventually critical, this situation is possible only if one (at least) of the centers $r_{\mathcal{U}_{1}}\left(\mathbf{a}_{0}\right)$ or $r_{\mathcal{U}_{2}}\left(\mathbf{a}_{0}\right)$ is at $\infty$ (Lemmas 2.21 and 1.6), or in the trivial case where $\mathcal{U}_{1}=\mathcal{U}_{2}, t_{1}=t_{2}$. Say $r_{\mathcal{U}_{2}}\left(\mathbf{a}_{0}\right)=\infty$, so $\mathcal{U}_{2}=\mathcal{H}_{\infty}$ and $r_{\mathcal{U}_{2}}(\mathbf{a})=\infty$ for every $\mathbf{a} \in \mathcal{P}_{n}$. Now, pulled back to the dynamical plane of $\mathbf{a}_{1}$ (through the holomorphic motion) the rays $R_{\mathbf{a}_{1}}^{\infty}\left(t_{2}\right)$ and $R_{\mathbf{a}_{1}}^{r_{\mathcal{U}_{1}}\left(\mathbf{a}_{1}\right)}\left(t_{1}\right)$ still land at a common point, by Corollary 3.10. This common point is $h_{n}^{\mathbf{a}_{1}}\left(H_{n}\left(\mathbf{a}_{1}\right)\right)=f_{\mathbf{a}_{1}}\left(-\mathbf{a}_{1}\right)$. This implies that $\mathcal{R}_{\infty}\left(t_{2}\right)$ lands at $\mathbf{a}_{1}$ (Lemma 2.26) and therefore that $\mathbf{a}_{1}=\mathbf{a}_{2}$.

The surjectivity follows from the same kind of arguments. The map is clearly surjective on the part of $I_{n+1}^{\mathbf{a}_{0}} \cap P_{n}^{\mathbf{a}_{0}}$ which is in $\overline{B_{\mathbf{a}_{0}}(\infty)}$ (the closure of the basin of $\infty$ ) by construction and by Lemma 2.26. Now let $z$ be a point in $P_{n}^{\mathbf{a}_{0}}$ on $I_{n+1}^{\mathbf{a}_{0}} \cap \widetilde{B}_{\mathbf{a}_{0}}$, so $z \in R_{\mathbf{a}_{0}}^{r}(t)$ for some center $r$ and angle $t$. Let $z_{1}$ be the landing point of this ray. By Remark 3.2 and Corollary 3.14 there is only one external ray in $I_{n+1}^{\mathbf{a}_{0}}$ that also lands at $z_{1}$, say $R_{\mathbf{a}_{0}}^{\infty}\left(t^{\prime}\right)$. Thus, the ray $\mathcal{R}_{\infty}\left(t^{\prime}\right)$ belongs to the para-graph $\mathcal{I}_{n+1}$ (by the surjectivity). It lands at a parameter $\mathbf{a}_{1}$ and there is only one ray in $\mathcal{I}_{n+1}$ that also lands at $\mathbf{a}_{1}$ (by the injectivity of $H_{n}$ ). Consider the simple arc formed by the union of this ray, $\left\{\mathbf{a}_{1}\right\}$ and $\mathcal{R}_{\infty}\left(t^{\prime}\right)$. Its image by $H_{n}$ must contain $z$, by the injectivity of $H_{n}$ and since there is no other branch of $I_{n+1}^{\mathbf{a}_{0}}$ at $z_{1}$. This reasoning extends to the parameters of $\mathcal{I}_{n+1}$ lying on equipotentials.

COROLLARY 3.18. - Let $\mathbf{a} \in \mathcal{P}_{n-1}$. The parameter a belongs to the annulus $\mathcal{P}_{n-1} \backslash \overline{\mathcal{P}}_{n}$ if and only if the critical value $f_{\mathbf{a}}(-\mathbf{a})$ lies in $P_{n-1}^{\mathbf{a}} \backslash \overline{C_{n}^{\mathbf{a}}}$ where $C_{n}^{\mathbf{a}}$ is the puzzle piece bounded by $h_{n-1}^{\mathbf{a}}\left(\partial P_{n}^{\mathbf{a}_{0}}\right)$.

Proof. - By definition, $\mathbf{a} \in \mathcal{P}_{n-1}$ implies that $f_{\mathbf{a}}(-\mathbf{a}) \in P_{n-1}^{\mathbf{a}}$. In the proof of Lemma 3.17 we showed that the piece $P_{n}^{\mathbf{a}}$ is bounded by $h_{n-1}^{\mathbf{a}}\left(\partial P_{n}^{\mathbf{a}_{0}}\right)$, for $\mathbf{a} \in \mathcal{P}_{n}$. Thus if $f_{\mathbf{a}}(-\mathbf{a})$ lies in $P_{n-1}^{\mathbf{a}} \backslash \overline{C_{n}^{\mathbf{a}}}$, then the parameter $\mathbf{a}$ is in $\mathcal{P}_{n-1} \backslash \overline{\mathcal{P}}_{n}$.

Conversely, suppose a belongs to $\mathcal{P}_{n-1} \backslash \overline{\mathcal{P}}_{n}$ and denote by $\mathcal{P}_{n}($ a) the new para-puzzle piece of depth $n$ containing a (if $\mathbf{a} \in \mathcal{X}_{n-1} \backslash \mathcal{X}_{n}$ the result is clear). We construct a continuous path $\mathbf{a}_{t} \subset \mathcal{P}_{n-1}$ joining $\mathbf{a}_{0}$ to $\mathbf{a}_{1}=\mathbf{a}$, crossing $\partial \mathcal{P}_{n}\left(\right.$ resp. $\left.\partial \mathcal{P}_{n}(\mathbf{a})\right)$ at exactly one point $\mathbf{a}_{t_{0}}\left(\right.$ resp. $\left.\mathbf{a}_{t_{1}}\right)$ on equipotentials of $\mathcal{I}_{n}$ and avoiding $\mathcal{I}_{n} \backslash\left\{\mathbf{a}_{t_{0}}, \mathbf{a}_{t_{1}}\right\}$. For this, we connect $\mathbf{a}_{0}$ by a path in $\mathcal{P}_{n}$ to a point $\mathbf{a}^{\prime} \in \mathcal{H}_{\infty} \cap \mathcal{P}_{n}$ then we follow the ray containing $\mathbf{a}^{\prime}$ and cross $\partial \mathcal{P}_{n}$ at an equipotential, we then take an equipotential contained in $\mathcal{X}_{n-1} \backslash \overline{\mathcal{X}_{n}}$ and join $\mathbf{a}_{1}$ by a ray entering $\mathcal{P}_{n}(\mathbf{a})$ and a piece of path, as before, inside $\mathcal{P}_{n}(\mathbf{a})$.

Thus, for $t<t_{0}$, the critical value $f_{\mathbf{a}_{t}}\left(-\mathbf{a}_{t}\right)$ belongs to the puzzle piece bounded by $h_{n-1}^{\mathbf{a}_{t}}\left(\partial P_{n}^{\mathbf{a}_{0}}\right)$. Moreover, since the parameter path $\mathbf{a}_{t}$ crosses $\mathcal{I}_{n}$ at $t=t_{0}$ on a ray (changing so the value of its potential) the critical value $f_{\mathbf{a}_{t}}\left(-\mathbf{a}_{t}\right)$ goes out of the piece bounded by $h_{n-1}^{\mathbf{a}}\left(\partial P_{n}^{\mathbf{a}_{0}}\right)$ when $t$ passes over $t_{0}$. Then Lemma 3.17 insures that, for $t_{0}<t<t_{1}$, the critical value does not cross $I_{n}^{\mathbf{a}_{t}}$ again. Hence the critical value $f_{\mathbf{a}_{t}}\left(-\mathbf{a}_{t}\right)$ is outside the piece bounded by $h_{n-1}^{\mathbf{a}_{t}}\left(\partial P_{n}^{\mathbf{a}_{0}}\right)$. Now at $t_{1}$, the critical value $f_{\mathbf{a}_{t_{1}}}\left(-\mathbf{a}_{t_{1}}\right)$ belongs to $I_{n}^{\mathbf{a}_{t_{1}}}$, but not to $h_{n-1}^{\mathbf{a}_{t_{1}}}\left(\partial P_{n}^{\mathbf{a}_{0}}\right)$ since $H_{n-1}\left(\mathbf{a}_{t_{1}}\right) \notin \partial P_{n}^{\mathbf{a}_{0}}$ by the injectivity of $H_{n}$ (see Lemma 3.17). Thus as before going inside $\mathcal{P}_{n}(\mathbf{a})$ along a ray, the critical value enters a new puzzle piece which is not bounded by $h_{n-1}^{\mathbf{a}_{t}}\left(\partial P_{n}^{\mathbf{a}_{0}}\right)$.

COROLLARY 3.19. - If $\overline{P_{n}^{\mathbf{a}_{0}}} \subset P_{n-1}^{\mathbf{a}_{0}}$ then $\overline{\mathcal{P}}_{n} \subset \mathcal{P}_{n-1}$.

Proof. - Assume, to get a contradiction, that $\partial \mathcal{P}_{n} \cap \partial \mathcal{P}_{n-1} \neq \emptyset$. In the graph $I_{0}^{\mathbf{a}_{0}}(\theta)$ two rays never converge to the same point, nor do they in $I_{n}^{\mathbf{a}_{0}}(\theta)$ by pullback, nor in $\mathcal{I}_{n}$ by isomorphism 
(or Lemma 2.26). Therefore, the intersection $\partial \mathcal{P}_{n} \cap \partial \mathcal{P}_{n-1}$ contains at least a part of external rays. Indeed, if it contains a part of internal ray it then also contains a part of external ray, so we only have to consider case of the external rays. Let $\mathbf{a}_{1}$ be such an intersection point contained in some ray $\mathcal{R}_{\infty}(t)$. So $d^{n} t \in\left\{d^{j} \eta, j \geqslant 0\right\}$ but also $d^{n-1} t \in\left\{d^{j} \eta, j \geqslant 0\right\}$. Through a path in $\mathcal{P}_{n}$ one can go from $\mathbf{a}_{1}$ to the "center" $\mathbf{a}_{0}$ of $\mathcal{P}_{n}$ without crossing the graph $\mathcal{I}_{n}$ (outside $\mathbf{a}_{1}$ ). Since the graph admits a holomorphic motion in $\mathcal{P}_{n}$, the critical value $f_{\mathbf{a}}(-\mathbf{a})$ enters the piece $P_{n}^{\mathbf{a}}$ which is bounded by $h_{n-1}^{\mathbf{a}}\left(\partial P_{n}^{\mathbf{a}_{0}}\right)$ (this is clear taking a path which starts by some part of equipotential). On the path and by holomorphic motion, the ray $R_{\mathbf{a}}^{\infty}(t) \cap X_{n}^{\mathbf{a}}$ is in the boundary $\partial P_{n}^{\mathbf{a}}=h_{n-1}^{\mathbf{a}}\left(\partial P_{n}^{\mathbf{a}_{0}}\right)$. Therefore $R_{\mathbf{a}_{0}}^{\infty}(t)$ belongs to $I_{n}^{\mathbf{a}_{0}}$. But, since $d^{n-1} t \in\left\{d^{j} \eta, j \geqslant 0\right\}$, the ray $R_{\mathbf{a}_{0}}^{\infty}(t)$ is also in $I_{n-1}^{\mathbf{a}_{0}}$. Therefore, $\partial P_{n}^{\mathbf{a}_{0}} \cap \partial P_{n}^{\mathbf{a}_{0}} \neq \emptyset$ which contradicts the hypothesis.

COROLlARY 3.20. - If $\partial \mathcal{P}_{n+1} \subset \mathcal{P}_{n}$, then $H_{n}$ induces a homeomorphism between $\partial \mathcal{P}_{n+1}$ and $\partial P_{n+1}^{\mathbf{a}_{0}}$.

Proof. - Since $H_{n}$ is not well defined on $\partial \mathcal{P}_{n}$ we need the assumption that $\partial \mathcal{P}_{n+1} \subset \mathcal{P}_{n}$. Then the result follows from Lemma 3.17.

\subsection{Graphs and renormalization}

In the dynamical plane, the graphs of Definition 3.1 are used to prove the following theorem (see [17]):

THEOREM [7,17]. - The boundary of $B_{\mathbf{a}}$, as well as of any connected component of $\widetilde{B}_{\mathbf{a}}$, is a Jordan curve for any $\mathbf{a} \in \mathbf{C}{ }^{7}$

It follows (by Remark 3.21) from Proposition 3.22 which is a formulation of Yoccoz' theorem in the context of the family $\left(f_{\mathbf{a}}\right)$.

Remark 3.21. - Let $p$ be a point in some region $U \subset$ C. If a sequence of disjoint annuli $A_{k}$ are homotopic in $U \backslash\{p\}$ and satisfy $\sum_{k \geqslant 0} \bmod A_{k}=\infty$ then the diameter of $U_{k}$, the connected component of $U \backslash A_{k}$ containing $p$, shrinks to 0 .

Proof. - This is a direct consequence of the following classical results (see [1]):

- Grötzsch inequality: $\bmod A \geqslant \sum_{i} \bmod A_{i}$ when $A_{i}$ are disjoint sub-annuli of $A$ homotopic to $A$;

- for any compact $K$ contained in a disk $D$, if the annulus $D \backslash K$ has infinite modulus, then $K$ is just a point.

Proposition 3.22. - Let $\mathbf{a} \in \mathcal{C} \backslash \mathbf{R}^{-}$. There exist $\epsilon= \pm 1$ and $l_{0}$ such that for $l \geqslant l_{0}$ the puzzle defined by $I_{0}^{\mathbf{a}}(\theta)$ with $\theta=\frac{\epsilon}{(d-1)^{l}-1}$ gives a sequence of non-degenerate annuli $A_{n_{i}}^{\mathbf{a}}$ satisfying:

1. for $i \geqslant 1, A_{n_{i}}^{\mathbf{a}}=P_{n_{i}}^{\mathbf{a}} \backslash \overline{P_{n_{i}+1}^{\mathbf{a}}}$; so $A_{n_{i}}^{\mathbf{a}}$ surrounds the critical value for $i \geqslant 1$ but maybe not for $i=0$;

2. $f_{\mathbf{a}}^{n_{i}-n_{0}}$ induces a non-ramified covering map from $\overline{A_{n_{i}}^{\mathbf{a}}}$ onto $\overline{A_{n_{0}}^{\mathbf{a}}}$;

3. either $\sum_{i \geqslant 0} \bmod A_{n_{i}}^{\mathbf{a}}=\infty\left(\right.$ where $\bmod A_{n_{i}}^{\mathbf{a}}$ denotes the modulus of $\left.A_{n_{i}}^{\mathbf{a}}\right)$ or there exists $k>1$ such that $f_{\mathbf{a}}^{k}: P_{n+k}^{\mathbf{a}} \rightarrow P_{n}^{\mathbf{a}}$ is a quadratic-like map for every large $n$.

The proof of this proposition can be found in [17] as a consequence of Lemma 2.9, Lemma 2.10 and Theorem 1.10 (Theorem of Yoccoz) in this article. Similar formulations can be found in $[7,13]$. It will be used several times later.

\footnotetext{
${ }^{7}$ For completeness, we will sketch its proof in Section 5.3.

$4^{\mathrm{e}}$ SÉRIE - TOME $40-2007-\mathrm{N}^{\circ} 6$
} 
DEFINITION 3.23. - A proper holomorphic map $f: U \rightarrow V$ is quadratic-like if $U, V$ are topological disks with $\bar{U} \subset V$ and if the degree of $f$ is 2 .

A map $f$ is said to be renormalizable if there exist disks $U, V$ and some integer $k>1$ such that $f^{k}: U \rightarrow V$ is quadratic-like and if the orbit of the unique critical point $x$ of the restriction $f_{\mid U}^{k}$ stays in $U$, i.e. $f^{k n}(x) \in U$ for all $n \geqslant 0$. The integer $k$ is called the period.

LEMMA 3.24. - The map $f_{\mathbf{a}}$ is renormalizable if and only if there exists $l_{1}>l_{0}$ such that for $l \geqslant l_{1}$ the second case of the alternative of Proposition 3.22 .3 occurs for the graphs $I_{0}^{\mathbf{a}}(\theta)$ defined by $\theta=\frac{\epsilon}{(d-1)^{l}-1}$.

Proof. - First we suppose that $f_{\mathbf{a}}$ satisfies the second case of the alternative of Proposition 3.22.3. Then, there exist $n_{0}$ and $k>1$ such that $f_{\mathbf{a}}^{k}: P_{n+k}^{\mathbf{a}} \rightarrow P_{n}^{\mathbf{a}}$ is a quadratic-like map for every $n \geqslant n_{0}$. Since $f_{\mathbf{a}}(-\mathbf{a})$ belongs to $P_{n}^{\mathbf{a}}$, the critical point $-\mathbf{a}$ belongs to $f_{\mathbf{a}}^{k-1}\left(P_{n+k}^{\mathbf{a}}\right)=$ $P_{n-1}^{\mathbf{a}}\left(f_{\mathbf{a}}^{k}(-\mathbf{a})\right)$ for every $n \geqslant n_{0}$ and the restriction $f_{\mathbf{a}}^{k-1}: P_{n+k}^{\mathbf{a}} \rightarrow P_{n-1}^{\mathbf{a}}\left(f_{\mathbf{a}}^{k}(-\mathbf{a})\right)$ is a homeomorphism. Denote by $x_{\mathbf{a}}$ the unique preimage of $-\mathbf{a}$ by the restriction. Since the pieces are nested, this preimage does not depend on $n$ and $x_{\mathbf{a}} \in P_{i}$ for every $i \geqslant k$. The point $x_{\mathbf{a}}$ is the critical point of the restriction $f_{\mathbf{a}}^{k}: P_{n+k}^{\mathbf{a}} \rightarrow P_{n}^{\mathbf{a}}$ for every $n \geqslant n_{0}$. To prove that $f_{\mathbf{a}}$ is renormalizable it is enough to see that if we fix some $n \geqslant n_{0}$, the image $f_{\mathbf{a}}^{k i}\left(x_{\mathbf{a}}\right)$ belongs to $P_{n+k}^{\mathbf{a}}$ for all $i \geqslant 0$. This follows from the fact that $x_{\mathbf{a}} \in P_{n+k+k i}^{\mathbf{a}}$ for every $i \geqslant 0$ so that $f_{\mathbf{a}}^{k i}\left(x_{\mathbf{a}}\right) \in P_{n+k}^{\mathbf{a}}$.

Now we assume that $f_{\mathbf{a}}$ is renormalizable, and that the first alternative of 3 of Proposition 3.22 does not hold. Let $K_{\mathrm{a}}$ denote its filled Julia set of the renormalization and $k$ the period: $K_{\mathbf{a}}=\bigcap_{i \geqslant 0}\left(f_{\mathbf{a}}^{k}\right)^{-i}(U)$ for $U$ as in Definition 3.23. We prove first that the intersection $K_{\mathbf{a}} \cap \partial B_{\mathbf{a}}$ contains at most one point. Assume that there are at least two points in this intersection but also that $K_{\mathbf{a}}$ is not contained in $\partial B_{\mathbf{a}}$. Then there is a bounded connected component in $\mathbf{C} \backslash\left(\partial B_{\mathbf{a}} \cup K_{\mathbf{a}}\right)$ so there are points on the boundary of this connected component (and also on $\partial B_{\mathbf{a}}$ ) which are not in $\partial B_{\mathbf{a}}(\infty)$; this is not possible for a polynomial. If $K_{\mathbf{a}} \subset \partial B_{\mathbf{a}}$ we would deduce by iteration that $\partial B_{\mathbf{a}}=K_{\mathbf{a}}$, since $\partial B_{\mathbf{a}}$ is a Jordan curve; this is not possible for a polynomial (namely for $f_{\mathbf{a}}^{k}$ ). Now we can prove that $K_{\mathbf{a}}$ is included in the puzzle pieces $P_{n}^{\mathbf{a}}(-\mathbf{a})$ as follows. If $K_{\mathbf{a}}$ is cut by $\partial P_{n}^{\mathbf{a}}(-\mathbf{a})$ there are some rays in $\tilde{B}_{\mathbf{a}}$ converging to points of $K_{\mathbf{a}}$, so by iteration some ray of the graph of depth 0 converges to a point of $K_{\mathbf{a}} \cap \partial B_{\mathbf{a}}$. The intersection point has to be fixed by $f_{\mathbf{a}}^{k}$ (else there are more than one point in the intersection). For $l_{1}>l_{0}$, the rays of the graphs defined for $l \geqslant l_{1}$ are not $k$-periodic so cannot converge to $K_{\mathbf{a}} \cap \partial B_{\mathbf{a}}$. Therefore $K_{\mathbf{a}}$ is included in all the puzzle pieces $P_{i}^{\mathbf{a}}(-\mathbf{a})$, so $f_{\mathbf{a}}^{k}: P_{n+k}^{\mathbf{a}} \rightarrow P_{n}^{\mathbf{a}}$ is quadratic-like and we are in the second case of the alternative of Proposition 3.22.3 for those graphs.

Definition 3.25. - A set $\mathbf{M}_{0}$ is a copy of $\mathbf{M}$ if there exist a homeomorphism $\chi$ and an integer $k>1$ (the period) such that

1. $\mathbf{M}_{0}=\chi^{-1}(\mathbf{M})$,

2. $\chi^{-1}(\partial \mathbf{M}) \subset \partial \mathcal{C}$ and for every $\mathbf{a} \in \mathbf{M}_{0}$,

3. $f_{\mathbf{a}}$ is renormalizable near the critical point $-\mathbf{a}$ with $f_{\mathbf{a}}^{k}$ topologically conjugated to $z^{2}+\chi(\mathbf{a})$ on neighbourhoods of the filled Julia sets.

PROPOSITION 3.26. - If $f_{\mathbf{a}_{0}}$ is renormalizable, $\mathbf{M}_{\mathbf{a}_{0}}=\bigcap_{n \geqslant 0} \mathcal{P}_{n}\left(\mathbf{a}_{0}\right)$ is a copy of $\mathbf{M}$.

Proof. - Since $f_{\mathbf{a}_{0}}$ is renormalizable, there exist $l_{1}>l_{0}$ such that the graphs defined in Lemma 3.24 satisfy the second case of the alternative of Proposition 3.22.3. We prove that $\left\{f_{\mathbf{a}}^{k}: P_{n}^{\mathbf{a}} \rightarrow P_{n-k}^{\mathbf{a}}, \mathbf{a} \in \mathcal{P}_{n}\left(\mathbf{a}_{0}\right)\right\}$ form a Mandelbrot-like family.

For $n \geqslant n_{0}$ and $\mathcal{P}_{n}=\mathcal{P}_{n}\left(\mathbf{a}_{0}\right)$, we consider the mapping $\mathbf{f}: \mathcal{W}^{\prime} \rightarrow \mathcal{W}$ defined by $\mathcal{W}=\{(\mathbf{a}, z) \mid$ $\left.\mathbf{a} \in \mathcal{P}_{n}, z \in P_{n-k}^{\mathbf{a}}\right\}, \mathcal{W}^{\prime}=\left\{(\mathbf{a}, z) \mid \mathbf{a} \in \mathcal{P}_{n}, z \in P_{n}^{\mathbf{a}}\right\}$ and $\mathbf{f}(\mathbf{a}, z)=\left(\mathbf{a}, f_{\mathbf{a}}^{k}(z)\right)$. They form an analytic family of quadratic-like maps in the sense of Douady and Hubbard [6, p. 304] since they satisfy the following three properties: 
- the map $\mathrm{f}: \mathcal{W}^{\prime} \rightarrow \mathcal{W}$ is holomorphic and proper;

- the holomorphic motion of the disk $P_{n}^{\mathbf{a}}$, resp. $P_{n-k}^{\mathbf{a}}$, is a homeomorphism between $\mathcal{W}^{\prime}$, resp. $\mathcal{W}$, and $\mathcal{P}_{n} \times \mathbf{D}$ which is fibered over $\mathcal{P}_{n}$ (since $\mathbf{a} \in \mathcal{P}_{n}$ );

- the projection $\overline{\mathcal{W}}^{\prime} \cap \mathcal{W} \rightarrow \mathcal{P}_{n}$ (i.e. the first coordinate) is proper, since $\overline{\mathcal{W}}^{\prime} \cap \mathcal{W}=\{(\mathbf{a}, z) \mid$ $\left.\mathbf{a} \in \mathcal{P}_{n}, z \in \overline{P_{n}^{\mathbf{a}}}\right\}$.

Let $\mathbf{M}_{\mathbf{f}}=\left\{\mathbf{a} \mid K\left(f_{\mathbf{a}}^{k}\right)\right.$ is connected $\}$ denote the connectedness locus of $\mathbf{f}$, where $K\left(f_{\mathbf{a}}^{k}\right)=$ $\bigcap_{i \geqslant 0}\left(f_{\mathbf{a}}^{k}\right)^{-i}\left(P_{n}^{\mathbf{a}}\right)$ denote its filled Julia set. Then $\mathbf{M}_{\mathbf{f}}$ coincides with $\mathbf{M}_{\mathbf{a}_{0}}$. Indeed, for $\mathbf{a} \in \mathbf{M}_{\mathbf{a}_{0}}$, the critical point $-\mathbf{a}$, and its orbit under $f_{\mathbf{a}}$, does never cross the graphs $I_{j}^{\mathbf{a}}(j \geqslant 0)$ since a belongs to every para-puzzle piece. Therefore the critical point $x_{\mathbf{a}}$ of $\left.f_{\mathbf{a}}^{k}\right|_{P_{n}^{\mathbf{a}}}$ never escapes the piece $P_{n}^{\mathbf{a}}$ (by iteration by $f_{\mathbf{a}}^{k}$ ). Hence $K\left(f_{\mathbf{a}}^{k}\right)$ is connected and $\mathbf{a} \in \mathbf{M}_{\mathbf{f}}$. Conversely, for $\mathbf{a} \in \mathcal{P}_{n} \backslash \overline{\mathcal{P}}_{n+1}$, the critical value belongs to $A_{n}^{\mathbf{a}}$ (by Corollary 3.18 ). Thus $f_{\mathbf{a}}^{k}\left(f_{\mathbf{a}}(-\mathbf{a})\right.$ ) is not in $P_{n-k}^{\mathbf{a}}$ and therefore the critical point of $f_{\mathbf{a}}^{k}$ escapes the domain; then the filled Julia set is not connected anymore so that $\mathbf{a} \notin \mathbf{M}_{\mathbf{f}}$.

Moreover, by Corollary 3.19 and Proposition 3.22, there exists a sequence $n_{i}$ such that $\overline{\mathcal{P}_{n_{i}+1}} \subset \mathcal{P}_{n_{i}}$. Thus $\mathbf{M}_{\mathbf{a}_{0}}$ is also the intersection of the closed pieces: $\mathbf{M}_{\mathbf{a}_{0}}=\bigcap_{n>0} \overline{\mathcal{P}}_{n}$ and therefore is compact.

Now, the theory of Mandelbrot-like families of Douady and Hubbard (see [6], Theorem II.2, Propositions II.14 and IV.21) gives a continuous map $\chi: \mathcal{P}_{n} \rightarrow \mathbf{C}$ such that the maps $f_{\mathbf{a}}^{k}$ and $z^{2}+\chi(\mathbf{a})$ are quasi-conformally conjugated on a neighbourhood of the filled Julia sets, for every $\mathbf{a} \in \mathcal{P}_{n}$.

Moreover, since $\mathbf{M}_{\mathbf{f}}$ is compact, the map $\chi$ induces a homeomorphism between $\mathbf{M}_{\mathbf{f}}$ and the Mandelbrot set $\mathbf{M}$ if we are in the following situation (see [6]): for a closed disk $\Delta \subset \mathcal{P}_{n}$ containing $\mathbf{M}_{\mathbf{f}}$ in its interior, the quantity $f_{\mathbf{a}}^{k}\left(x_{\mathbf{a}}\right)-x_{\mathbf{a}}$, (where $x_{\mathbf{a}}$ denotes the critical point of $\left.f_{\mathbf{a}}^{k}\right|_{P_{n}^{\mathbf{a}}}$ should turn exactly once around 0 when a describes $\partial \Delta$. We verify this property now.

Take some piece $\mathcal{P}_{p}\left(\mathbf{a}_{0}\right)=\Delta$, compactly contained $\mathcal{P}_{n}\left(\mathbf{a}_{0}\right)$ (see Corollary 3.19). It is a topological disk containing $\mathcal{M}_{\mathbf{a}_{0}}$ in its interior. To compute the degree on $\partial \Delta$ of $\gamma(\mathbf{a})=$ $f_{\mathbf{a}}^{k}\left(x_{\mathbf{a}}\right)-x_{\mathbf{a}}$ we make a homotopy of this curve $\gamma$ to the curve $H_{p-1}(\mathbf{a})-x_{\mathbf{a}_{0}}$ as follows, where $H_{p}$ is as in Lemma 3.17. The critical point $x_{\mathbf{a}}$ of $f_{\mathbf{a}}^{k}$ satisfies that $f_{\mathbf{a}}^{k-1}\left(x_{\mathbf{a}}\right)=-\mathbf{a}$, so $\gamma(\mathbf{a})=$ $f_{\mathbf{a}}(-\mathbf{a})-x_{\mathbf{a}}$. Let $h(\mathbf{a}, z)=h_{p-1}(\mathbf{a}, z)-x_{\mathbf{a}}$, then $\gamma(\mathbf{a})=h\left(\mathbf{a}, H_{p-1}(\mathbf{a})\right)$. Assume that $\mathcal{P}_{p-1}$ is a round disk (if not use a conformal representation); then the homotopy is simply $G(t, \mathbf{a})=$ $h\left(\mathbf{a}_{0}+t\left(\mathbf{a}-\mathbf{a}_{0}\right), H_{p-1}(\mathbf{a})\right)$ joining $G(0, \mathbf{a})=H_{p-1}(\mathbf{a})-x_{\mathbf{a}_{0}}$ and $G(1, \mathbf{a})=f_{\mathbf{a}}(-\mathbf{a})-x_{\mathbf{a}}$.

Since $H_{p-1}$ is a homeomorphism from $\partial \mathcal{P}_{p}$ to $\partial P_{p}^{\mathbf{a}_{0}}$ (piece that surrounds $x_{\mathbf{a}_{0}}$ ), the degree of $H_{p-1}(\mathbf{a})-x_{\mathbf{a}_{0}}$ around 0 is exactly 1 , when a describes $\partial \mathcal{P}_{p}$.

PROPOSITION. - 1. If $f_{\mathbf{a}}$ has a periodic point $x \neq 0$ of multiplier $\rho$ with $|\rho| \leq 1$, then $f_{\mathbf{a}}$ is renormalizable near $x$ and $\mathbf{a}$ belongs to a copy of $\mathrm{M}$.

Proof. - Since $x \neq 0$, it is not in $\widetilde{B}_{\mathbf{a}}$ and, since it is not eventually repelling, it is not on any of the graphs. So we can consider the sequence $\left(P_{n}^{\mathbf{a}}(x)\right)$ of puzzle pieces containing $x$. Since $x$ is periodic, this sequence of pieces is periodic i.e., $f_{\mathbf{a}}^{k}\left(P_{n+k}^{\mathbf{a}}(x)\right)=P_{n}^{\mathbf{a}}(x)$ for any large $n$ and for some $k>1$. Choose the smallest $k$ with this property. There exists some $i \leqslant k$ such that the critical point $-\mathbf{a}$ belongs to the piece $P_{n}^{\mathbf{a}}\left(f_{\mathbf{a}}^{i}(x)\right)$, for every sufficiently large $n$. Otherwise the map $f_{\mathbf{a}}^{k}: P_{n+k}^{\mathbf{a}}(x) \rightarrow P_{n}^{\mathbf{a}}(x)$ would be invertible and its inverse $g: P_{n}^{\mathbf{a}}(x) \rightarrow P_{n}^{\mathbf{a}}(x)$ either would be an automorphism or has an attracting fixed point (by Schwarz' lemma). This is not possible since on the one hand $\left|g^{\prime}(x)\right| \geqslant 1$ and on the other hand the sequence $P_{n}^{\mathbf{a}}(x)$ is strictly decreasing $\left(\exists n \mid P_{n+k}^{\mathbf{a}}(x)=g\left(P_{n}^{\mathbf{a}}(x)\right) \neq P_{n}^{\mathbf{a}}(x)\right)$. This integer $i$ is independent of $n$ since the pieces $P_{n}^{\mathbf{a}}\left(f_{\mathbf{a}}^{j}(-\mathbf{a})\right)$ are disjoint for $j<k$. Therefore the map $f_{\mathbf{a}}^{k}: P_{n+k}^{\mathbf{a}}\left(f_{\mathbf{a}}^{i}(x)\right) \rightarrow P_{n}^{\mathbf{a}}\left(f_{\mathbf{a}}^{i}(x)\right)$ is quadratic-like. Hence we are in the situation of Lemma 3.24 where we proved that a belongs to a copy of the Mandelbrot set $\mathbf{M}$ (see Proposition 3.26).

$4^{\mathrm{e}}$ SÉRIE - TOME $40-2007-\mathrm{N}^{\circ} 6$ 
COROLLARY. - 1. Any bounded hyperbolic component either is a connected component of $\mathcal{H}$ or a hyperbolic component of a copy of $\mathbf{M}$.

Proof. - Let $\mathcal{U}$ be a hyperbolic component which is not in $\mathcal{H}$. For $\mathbf{a} \in \mathcal{U}$, the map $f_{\mathbf{a}}$ has an attracting periodic cycle, which is not the fixed point 0 . Thus we are in the situation of Proposition 1 so that the parameter a belongs to a copy of $\mathbf{M}$.

COROLLARY. - 2. If $f_{\mathbf{a}}$ has a periodic point $x$ of multiplier $\lambda=e^{2 i \pi \theta}$ with $\theta \in \mathbf{R} \backslash \mathbf{Q}$, then $f_{\mathbf{a}}$ is linearizable near $x$ if and only if $\theta \in \mathcal{B}$. Moreover, if $\theta \notin \mathcal{B}$ there exist periodic cycles in any neighbourhood of $x$.

Proof. - The map $f_{\mathbf{a}}$ is renormalizable by Proposition 1 . So there is a homeomorphism that conjugates $f_{\mathbf{a}}^{k}$ to a quadratic polynomial $z^{2}+\chi(\mathbf{a})$ on a neighbourhood of its Julia set (see [6]). The multipliers at the fixed points are the same by Naïshul's Theorem (see [15]). So the result follows from Yoccoz' and Brjuno's work (see [20]).

\section{Local connectivity}

Fix $\mathbf{a}_{0} \in \partial \mathcal{C} \cap \mathcal{S}$. Take $l_{0} \geqslant l_{1}$ given by Proposition 3.22 and Lemma 3.24, and $\theta \in$ $\left\{ \pm \frac{1}{(d-1)^{l}-1}\right\}$ with $l \geqslant l_{0}$.

Recall that the sequence of graphs $I_{n}^{\mathbf{a}_{0}}(\theta)$ and the para-graph $\mathcal{I}_{n}(\theta)$ associated in Definition 3.5 satisfy the following properties:

- The sequence of puzzle pieces $P_{n}^{\mathbf{a}_{0}}$ containing the critical value is well-defined since the critical value $f_{\mathbf{a}_{0}}\left(-\mathbf{a}_{0}\right)$ is on none of the graphs $I_{n}^{\mathbf{a}_{0}}, n \geqslant 0$.

- The sequence of para-puzzle pieces $\left(\mathcal{P}_{n}\right)_{n \in \mathbf{N}}$ containing $\mathbf{a}_{0}$ is well-defined by Lemma 3.17 (since the parameter $\mathbf{a}_{0}$ also never belongs to a graph $\mathcal{I}_{n}$ ).

- There exists a sequence of (non-degenerate) annuli $\left(A_{n_{i}}^{\mathbf{a}_{0}}\right)_{i \in \mathbf{N}}$ such that, for $i \geqslant 1, A_{n_{i}}^{\mathbf{a}_{0}}=$ $P_{n_{i}}^{\mathbf{a}_{0}} \backslash \overline{P_{n_{i}+1}^{\mathbf{a}_{0}}}$ (so surrounds the critical value $f_{\mathbf{a}_{0}}\left(-\mathbf{a}_{0}\right)$ ) and the map $f_{\mathbf{a}_{0}}^{n_{i}-n_{0}}: \overline{A_{n_{i}}^{\mathbf{a}_{0}}} \rightarrow \overline{A_{n_{0}}^{\mathbf{a}_{0}}}$ induces a non-ramified covering map (Proposition 3.22).

- The annuli $\mathcal{A}_{n_{i}}=\mathcal{P}_{n_{i}} \backslash \overline{\mathcal{P}_{n_{i}+1}}$ are non-degenerate (Corollary 3.19) and surround $\mathbf{a}_{0}$.

\subsection{Tools for proving local connectivity: estimation of moduli and connectivity questions}

The next proposition follows from Shishikura's trick to compare moduli of annuli.

Proposition 4.1. - There exists a constant $K>1$ such that, for $i \geqslant 0$,

$$
\frac{1}{K} \bmod A_{n_{i}}^{\mathbf{a}_{0}} \leqslant \bmod \mathcal{A}_{n_{i}} \leqslant K \bmod A_{n_{i}}^{\mathbf{a}_{0}}
$$

Proof. - The idea is to get a $K$-quasi-conformal homeomorphism between $\mathcal{A}_{n_{i}}$ and $A_{n_{i}}^{\mathbf{a}_{0}}$ extending the map $H_{n_{i}}$ (via Słodkovksi's theorem and the dynamical covering).

Fix $n \in\left\{n_{i}, i \geq 0\right\}$ and let $d_{n}$ be the degree of $f_{\mathbf{a}_{0}}^{n-n_{0}}: A_{n}^{\mathbf{a}_{0}} \rightarrow A_{n_{0}}^{\mathbf{a}_{0}}$. For every $\mathbf{a} \in \mathcal{P}_{n}$ we define, for $m \leqslant n$, the dynamical annuli $A_{m}^{\mathrm{a}}$, "motion" of $A_{m}^{\mathrm{a}_{0}}$, by the connected component of $P_{m}^{\mathbf{a}} \backslash h_{m}^{\mathbf{a}}\left(\partial P_{m+1}^{\mathbf{a}_{0}}\right)$ that intersects $I_{m+1}^{\mathbf{a}}$ in its interior. By definition (Lemma 3.16) the following diagram is commutative for $\mathbf{a} \in \mathcal{P}_{n}$.

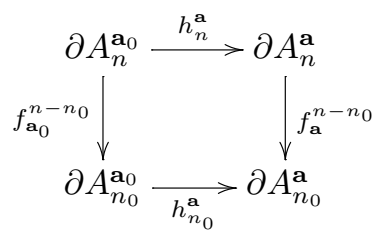


Thus $f_{\mathbf{a}}^{n-n_{0}}$ maps $\overline{A_{n}^{\mathbf{a}}}$ to $\overline{A_{n_{0}}^{\mathbf{a}}}$ and induces a non-ramified covering. Indeed, the critical value $f_{\mathbf{a}}(-\mathbf{a})$ remains outside $I_{n}^{\mathbf{a}}$ so that the critical point $-\mathbf{a}$ and all its preimages cannot enter $\overline{A_{n}^{\mathbf{a}}}$. At depth $n_{0}$ we extend the holomorphic motion $h_{n_{0}}: \mathcal{P}_{n_{0}} \times \partial A_{n_{0}}^{\mathbf{a}_{0}} \rightarrow \overline{\mathbf{C}}$ by Słodkowski's Theorem [19] to a holomorphic motion $\widetilde{h}_{n_{0}}: \mathcal{P}_{n_{0}} \times \overline{\mathbf{C}} \longrightarrow \overline{\mathbf{C}}$. For every $\mathbf{a} \in \mathcal{P}_{n_{0}}$, the map $\widetilde{h}_{n_{0}}^{\mathbf{a}}$ is a $K_{\mathbf{a}}$-quasi-conformal homeomorphism, with $K_{\mathbf{a}}=\frac{1+|\phi(\mathbf{a})|}{1-|\phi(\mathbf{a})|}$ where $\phi: \mathcal{P}_{n_{0}} \rightarrow \mathbf{D}$ is a conformal representation sending $\mathbf{a}_{0}$ to 0 . For every $\mathbf{a} \in \mathcal{P}_{n}$ the homeomorphism $\widetilde{h}_{n_{0}}^{\mathbf{a}}: \overline{A_{n_{0}}^{\mathbf{a}_{0}}} \rightarrow \overline{A_{n_{0}}^{\mathbf{a}}}$ liftsvia the holomorphic covering maps $f_{\mathbf{a}_{0}}^{n-n_{0}}$ and $f_{\mathbf{a}}^{n-n_{0}}$-to a quasi-conformal homeomorphism $\widetilde{h}_{n}^{\mathbf{a}}: \overline{A_{n}^{\mathbf{a}_{0}}} \longrightarrow \overline{A_{n}^{\mathbf{a}}}$ with the same dilatation $K_{\mathbf{a}}$. Moreover the identity $f_{\mathbf{a}}^{n-n_{0}} \circ h_{n}^{\mathbf{a}}=h_{n_{0}}^{\mathbf{a}} \circ f_{\mathbf{a}_{0}}^{n-n_{0}}$ ensures that the map $\widetilde{h}_{n}: \mathcal{P}_{n} \times \overline{A_{n}^{\mathbf{a}}} \rightarrow \overline{\mathbf{C}},(\mathbf{a}, z) \mapsto \widetilde{h}_{n}^{\mathbf{a}}(z)$, is a holomorphic motion that extends $h_{n}$. From Corollary 3.18 we know that a belongs to $\mathcal{A}_{n}$ if and only if $f_{\mathbf{a}}(-\mathbf{a})$ belongs to $A_{n}^{\mathbf{a}}$ so that a belongs to $\overline{\mathcal{A}_{n}}$ if and only if $f_{\mathbf{a}}(-\mathbf{a})$ belongs to $\overline{A_{n}^{\mathbf{a}}}$ or equivalently a belongs to $\mathcal{A}_{n} \cup \partial \mathcal{P}_{n+1}$ if and only if $\left(\widetilde{h}_{n}^{\mathbf{a}}\right)^{-1}\left(f_{\mathbf{a}}(-\mathbf{a})\right) \in A_{n}^{\mathbf{a}_{0}} \cup \partial P_{n+1}^{\mathbf{a}_{0}}$. Therefore the following map $\widetilde{H}_{n}$ is well-defined

$$
\widetilde{H}_{n}:\left\{\begin{aligned}
\mathcal{A}_{n} \cup \partial \mathcal{P}_{n+1} & \longrightarrow A_{n}^{\mathbf{a}_{0}} \cup \partial P_{n+1}^{\mathbf{a}_{0}}, \\
\mathbf{a} & \longmapsto \widetilde{H}_{n}(\mathbf{a})=\left(\widetilde{h}_{n}^{\mathbf{a}}\right)^{-1}\left(f_{\mathbf{a}}(-\mathbf{a})\right) .
\end{aligned}\right.
$$

From [6, IV.3] the map $\widetilde{H}_{n}$ is $K_{n}$-quasi-regular with $K_{n}=\sup \left\{K_{\mathbf{a}}, \mathbf{a} \in \mathcal{P}_{n}\right\}$ (see also [18]). Moreover $\widetilde{H}_{n}$ is a bijection since it agrees with $H_{n}$ on $\partial \mathcal{P}_{n+1}$ (Lemma 3.17). Therefore $\widetilde{H}_{n}$ is a $K$-quasi-conformal homeomorphism from $\mathcal{A}_{n}$ to $A_{n}^{\mathbf{a}_{0}}$ with $K=\sup \left\{K_{\mathbf{a}}, \mathbf{a} \in \overline{\mathcal{P}}_{n_{0}+1}\right\}<+\infty$. The result then follows.

In the rest of this subsection we prove the connectedness of the intersection of the para-puzzle pieces with $\partial \mathcal{C}$ and $\partial \mathcal{U}$.

LEMMA 4.2. - Let $\mathcal{U}$ be a connected component of $\mathcal{H}$. For every $n \geqslant 0$, the intersection of $\mathcal{U}$ and $\overline{\mathcal{P}}_{n}$ is a sector of $\mathcal{U}$ bounded by

$$
\partial \mathcal{P}_{n} \cap \mathcal{U}=\left(\mathcal{X}_{n} \cap\left(\mathcal{R}_{\mathcal{U}}\left(t_{n}\right) \cup \mathcal{R}_{\mathcal{U}}\left(t_{n}^{\prime}\right)\right)\right) \cup \widetilde{\mathcal{E}}_{\mathcal{U}}\left(v_{n}\right),
$$

where $\widetilde{\mathcal{E}}_{\mathcal{U}}\left(v_{n}\right)$ is a part of the equipotential $\mathcal{E}_{\mathcal{U}}\left(v_{n}\right)$ and $t_{n} \leqslant t_{n}^{\prime}$. Moreover, as $n$ tends to infinity, $v_{n} \rightarrow 0$ and $t_{n}, t_{n}^{\prime}$ converge to a common value.

Proof. - Since every ray of $\partial \mathcal{P}_{n} \cap \mathcal{U}$ is associated to an external ray, it is not possible to have more than two rays in $\partial \mathcal{P}_{n} \cap \mathcal{U}$ (two consecutive internal rays are connected by a section of equipotential and similarly for two consecutive external rays). So $t_{n}, t_{n}^{\prime}$ are consecutive angles in $\Theta_{n}$. We prove that $t_{n}-t_{n}^{\prime} \rightarrow 0$ for $\mathcal{H}_{0}$; the proof is the same for any $\mathcal{U}$. By definition of the graphs, any puzzle piece of depth 0 intersects $\mathcal{H}_{0}$ under an angular sector of width less than $1 / d$. Therefore the puzzle pieces of greater depth have rays in $\mathcal{H}_{0}$ whose angles are consecutive angles divided $d-1$. Thus $\left|t_{n}^{\prime}-t_{n}\right| \leqslant \frac{1}{d(d-1)^{n}}$, so $\lim t_{n}=\lim t_{n}^{\prime}=t$ (since both sequences are monotone).

LEMMA 4.3. - For every connected component $\mathcal{U}$ of $\mathcal{H}$, the intersection $\overline{\mathcal{P}}_{n} \cap \partial \mathcal{U}$ is a connected set for all $n \geqslant 0$.

Proof. - From Lemma 4.2, we know that $\partial \mathcal{U} \cap \overline{\mathcal{P}}_{n}$ is just the decreasing intersection of the compact connected sets $\overline{\Phi_{\mathcal{U}}\left(S_{k}^{n}\right)}$ where $S_{k}^{n}$ is the sector in the disk between the angles $t_{n}$ and $t_{n}^{\prime}$ and of potential less than $v_{k}^{n}$, where $\left(v_{k}^{n}\right)_{k \in \mathbf{N}}$ is a sequence which tends to 0 with $k$. Therefore it is compact and connected.

LEMmA 4.4. - For every $n \geqslant 0$, the intersection $\overline{\mathcal{P}}_{n} \cap \partial \mathcal{C}$ is connected.

$4^{\text {e }}$ SÉRIE - TOME $40-2007-\mathrm{N}^{\circ} 6$ 
Proof. - The property of the para-puzzle pieces we use here is to be a disk whose boundary is a succession of arcs of the following form: a part of an equipotential in $\mathcal{H}_{\infty}$ followed by a part of a ray in $\mathcal{X}_{n}$ converging to a point of $\partial \mathcal{C}$ and another part of a ray in $\mathcal{X}_{n}$ followed by a part of an equipotential in $\mathcal{H}$ (by Remark 3.2 and Lemma 3.17). We denote the property by $(*)$. Fix $n$ and let $\mathcal{G}_{n}$ be the bounded connected component of $\overline{\mathbf{C}} \backslash \mathcal{E}_{\infty}\left(1 / d^{n}\right)$. Consider $B(k)$ the set of disks $\mathcal{D} \subset \overline{\mathcal{G}}_{n}$ satisfying Property $(*)$ and such that $\mathcal{G}_{n} \backslash \mathcal{D}$ has $k$ connected components.

We prove by recurrence on $k$ that for any disk $\mathcal{D} \in B(k), \overline{\mathcal{D}} \cap \partial \mathcal{C}$ is connected.

Let $\mathcal{D} \in B(1)$, we prove by contradiction that $\overline{\mathcal{D}} \cap \partial \mathcal{C}$ is connected. Let $V$ be the complement $\mathcal{G}_{n} \backslash \overline{\mathcal{D}}$. Since $\mathcal{D}$ belongs to $B(1)$ there are only two parts of equipotentials in its boundary: one part of $\mathcal{E}_{\infty}\left(1 / d^{n}\right)$ and one part of an equipotential in a component $\mathcal{U}$ of $\mathcal{H}$. The complement $V$ has the same property. Therefore the intersection $\partial V \cap \mathcal{C}$ is reduced to the landing points of the external rays so is included into $\partial \mathcal{U} \cap \mathcal{C}$. Assume now that $\overline{\mathcal{D}} \cap \partial \mathcal{C}$ is not connected: $\overline{\mathcal{D}} \cap \partial \mathcal{C}=A \sqcup B$ where $A$ and $B$ are non-empty, closed and disjoint. The intersection $\overline{\mathcal{D}} \cap \partial \mathcal{U}$ is connected (it is the intersection of a decreasing sequence of connected compacts as in the previous Lemma 4.3) so we can assume that it is contained in $A$. Therefore $A^{\prime}=(V \cap \partial \mathcal{C}) \cup A$ is closed since the closure of $V \cap \partial \mathcal{C}$ is included in $A$. Moreover $A^{\prime}$ is disjoint from $B$ and $A^{\prime} \sqcup B=\partial \mathcal{C}$. This contradicts the fact that $\partial \mathcal{C}$ is connected.

Now fix some integer $k \geqslant 1$. Assume that we have proved the result for $B(i)$ with $i \leqslant k$. Take $\mathcal{D}$ a disk of $B(k+1)$. There exists at least a connected component, $\mathcal{V}$, of $\mathcal{G}_{n} \backslash \overline{\mathcal{D}}$ whose boundary intersects $\mathcal{E}_{\infty}\left(1 / d^{n}\right)$ under exactly one component. Then $\mathcal{D} \cup \overline{\mathcal{V}}$ is a disk in $B(k)$. So, with the same argument used before for $k=1$, if $\overline{\mathcal{D}} \cap \partial \mathcal{C}$ is not connected then $(\overline{\mathcal{D}} \cup \overline{\mathcal{V}}) \cap \partial \mathcal{C}$ is not connected neither. This gives the contradiction.

Now we can conclude in the non-renormalizable case.

LEMMA 4.5. - If the map $f_{\mathbf{a}_{0}}$ is not renormalizable, then $\partial \mathcal{C}$ and $\partial \mathcal{U}$ are locally connected at $\mathbf{a}_{0}$, where $\mathcal{U}$ is any connected component of $\mathcal{H}$.

Proof. - By Lemma 3.24 if the map $f_{\mathbf{a}_{0}}$ is not renormalizable it satisfies the first alternative of Proposition 3.22.3. The sequence of annuli considered in Proposition 3.22 has the property that $\sum_{i \geq 0} \bmod A_{n_{i}}^{\mathbf{a}_{0}}=\infty$. Hence $\sum_{i \geq 0} \bmod \mathcal{A}_{n_{i}}=\infty$ by Proposition 4.1. Thus the diameter of $\mathcal{P}_{n_{i}}$ shrinks to 0 by Remark 3.21.

Finally, $\overline{\mathcal{P}_{n_{i}}} \cap \partial \mathcal{U}$, resp. $\overline{\mathcal{P}_{n_{i}}} \cap \partial \mathcal{C}$, form a basis of connected neighbourhoods of $\mathbf{a}_{0}$ in $\partial \mathcal{U}$, resp. in $\partial \mathcal{C}$, by Lemma 4.3 , resp. Lemma 4.4 (since $\mathcal{P}_{n_{i}}$ is a neighbourhood of $\mathbf{a}_{0}$ ).

Corollary 4. - $\partial \mathcal{C}$ is locally connected at $\mathbf{a}_{0}$ as soon as $\mathbf{a}_{0}$ does not belong to a copy of $\mathrm{M}$.

Proof. - By Proposition 3.26 we are in the first alternative of Proposition 3.22.3, so the result follows from Lemma 4.5 .

\subsection{Local connectivity of $\partial \mathcal{H}_{0}$ and Wakes of $\mathcal{H}_{0}$}

Proposition 4.6. - Let $\mathbf{a}_{0} \in \partial \mathcal{H}_{0} \cap \mathcal{S}$. If $f_{\mathbf{a}_{0}}$ is renormalizable of period $k$, there exist $k$-periodic angles $t$ (under multiplication by $d-1$ ) and $\zeta, \zeta^{\prime}$ (under multiplication by $d$ ) such that the rays $\mathcal{R}_{0}(t), \mathcal{R}_{\infty}(\zeta)$ and $\mathcal{R}_{\infty}\left(\zeta^{\prime}\right)$ converge to $\mathbf{a}_{0}$. Moreover the curve $\mathcal{R}_{\infty}(\zeta) \cup \mathcal{R}_{\infty}\left(\zeta^{\prime}\right) \cup$ $\left\{\mathbf{a}_{0}\right\}$ separates $\mathbf{M}_{\mathbf{a}_{0}} \backslash\left\{\mathbf{a}_{0}\right\}$ from $\mathcal{H}_{0}$ where $\mathbf{M}_{\mathbf{a}_{0}}=\bigcap_{n \geqslant 0} \mathcal{P}_{n}\left(\mathbf{a}_{0}\right)$. In the dynamical plane, the rays $R_{\mathbf{a}_{0}}^{0}(t), R_{\mathbf{a}_{0}}^{\infty}(\zeta), R_{\mathbf{a}_{0}}^{\infty}\left(\zeta^{\prime}\right)$ converge to the same point which is a parabolic periodic point and $R_{\mathbf{a}_{0}}^{\infty}(\zeta) \cup R_{\mathbf{a}_{0}}^{\infty}\left(\zeta^{\prime}\right) \cup\left\{\mathbf{a}_{0}\right\}$ separates $f_{\mathbf{a}_{0}}\left(-\mathbf{a}_{0}\right)$ from $B_{\mathbf{a}_{0}}$.

Proof. - The para-puzzle piece $\mathcal{P}_{n}=\mathcal{P}_{n}\left(\mathbf{a}_{0}\right)$ intersects $\mathcal{H}_{0}$ since it is a neighbourhood of $\mathbf{a}_{0} \in \partial \mathcal{H}_{0}$. In particular, its boundary $\partial \mathcal{P}_{n}$ contains two rays in $\mathcal{H}_{0}$, say $\mathcal{R}_{0}\left(t_{n}\right), \mathcal{R}_{0}\left(t_{n}^{\prime}\right)$, landing 


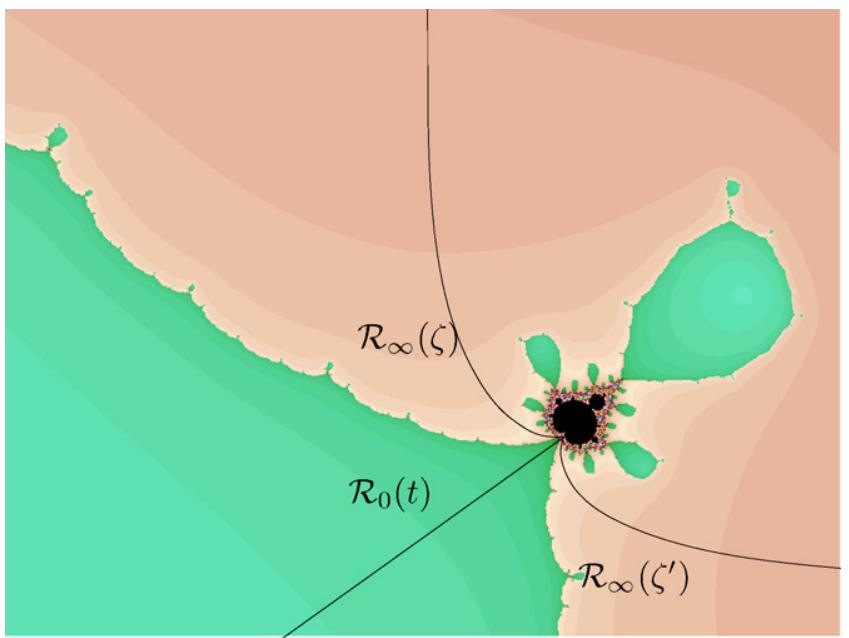

Fig. 8. Separation of $\mathbf{M}_{\mathbf{a}_{0}}$ (copy of $\mathbf{M}$ ) from $\mathcal{H}_{0}$ by rays.

at parameters called $\mathbf{a}_{n}, \mathbf{a}_{n}^{\prime}$ respectively and two external rays say $\mathcal{R}_{\infty}\left(\zeta_{n}\right), \mathcal{R}_{\infty}\left(\zeta_{n}^{\prime}\right)$ also landing at $\mathbf{a}_{n}$ and $\mathbf{a}_{n}^{\prime}$ respectively. The sequences of angles $\left(t_{n}\right),\left(t_{n}^{\prime}\right)$ converge to some common value $t$ with $t_{n} \leqslant t \leqslant t_{n}^{\prime}$ (see Lemma 4.2). Moreover, the sequence of intervals $\left(\zeta_{n}, \zeta_{n}^{\prime}\right)$ is decreasing, so the angles $\zeta_{n}, \zeta_{n}^{\prime}$ converge to some values $\zeta, \zeta^{\prime}$.

The boundary of the dynamical puzzle pieces $P_{n}^{\mathbf{a}}$ is given for the subsequence $n_{i}$ such that $\partial \mathcal{P}_{n_{i}+1} \subset \mathcal{P}_{n_{i}}$ by the bijection described in Corollary 3.20. It contains parts of the rays $R_{\mathbf{a}_{0}}^{0}\left(t_{n_{i}}\right), R_{\mathbf{a}_{0}}^{0}\left(t_{n_{i}}^{\prime}\right)$ and $R_{\mathbf{a}_{0}}^{\infty}\left(\zeta_{n_{i}}\right), R_{\mathbf{a}_{0}}^{\infty}\left(\zeta_{n_{i}}^{\prime}\right)$ landing at points $z_{n_{i}}\left(\mathbf{a}_{0}\right), z_{n_{i}}^{\prime}\left(\mathbf{a}_{0}\right)$ respectively. Since $f_{\mathbf{a}_{0}}$ is renormalizable, the puzzle pieces are "periodic" i.e., $f_{\mathbf{a}_{0}}^{k}\left(\overline{P_{n+k}^{\mathbf{a}_{0}}}\right)=\overline{P_{n}^{\mathbf{a}_{0}}}$ by Lemma 3.24. Hence the angles satisfy the relation $(d-1)^{k} \theta_{n+k}=\theta_{n} \bmod 1$ for the rays in $B_{\mathbf{a}}$ and $d^{k} \theta_{n+k}=\theta_{n} \bmod 1$ for the external rays. This is clear for the rays in $B_{\mathbf{a}_{0}}$ since there are only two rays in $\partial P_{n}^{\mathbf{a}_{0}} \cap B_{\mathbf{a}_{0}}$ and it follows for the external rays since they converge to the same points $z_{n}, z_{n}^{\prime}$. Then the angles $t, \zeta, \zeta^{\prime}$ satisfy the same relations and therefore are of the form $\frac{p}{(d-1)^{k}-1}, \frac{q}{d^{k}-1}, \frac{q^{\prime}}{d^{k}-1}$ respectively.

We prove now that $\mathcal{R}_{0}(t), \mathcal{R}_{\infty}(\zeta)$ and $\mathcal{R}_{\infty}\left(\zeta^{\prime}\right)$ converge to $\chi_{\mathbf{a}_{0}}^{-1}(1 / 4)$ where $\chi_{\mathbf{a}_{0}}$ is the homeomorphism that maps $\mathbf{M}_{\mathbf{a}_{0}}$ to $\mathbf{M}$. The proof is the same for the three rays, we do it for $\mathcal{R}_{0}(t)$. The ray $\mathcal{R}_{0}(t)$ converges, since $t$ is rational, to some parameter $\mathbf{a}_{1}$. For every $n \geqslant 0$ the part of the ray $\mathcal{R}_{0}(t) \cap \mathcal{X}_{n}$ is in $\mathcal{P}_{n}$, because of the bijection between $\partial \mathcal{P}_{n_{i}}$ and $\partial P_{n_{i}}^{\mathbf{a}_{0}}$ and of Lemma 4.2. Thus $\mathbf{a}_{1}$ belongs to $\mathbf{M}_{\mathbf{a}_{0}}$. In the dynamical plane, the ray $R_{\mathbf{a}_{1}}^{0}(t)$ lands at a periodic point $z_{1}$, since $t$ is periodic. Its period, say $k$, is the period of $t$ (two rays in $B_{\mathbf{a}_{1}}$ cannot land at the same point). The point $z_{1}$ cannot be eventually critical since $t$ is periodic. It is parabolic by Lemma 1.4 since the ray $R_{\mathbf{a}_{1}}^{0}(t)$ is not stable in any neighbourhood of $\mathbf{a}_{1}$ by the following remarks. For $\mathbf{a} \in \mathcal{R}_{0}(t)$ near $\mathbf{a}_{1}$, the critical value is on $R_{\mathbf{a}}^{0}(t)=R_{\mathbf{a}}^{0}\left((d-1)^{k} t\right)$. Moreover the critical point $-\mathbf{a}$ is in $f_{\mathbf{a}}^{k-1}\left(P_{n+k}^{\mathbf{a}}\right)$ since $f_{\mathbf{a}_{1}}$ is renormalizable and $\mathbf{a}$ is very close to $\mathbf{a}_{1}$. So $-a$ is on the preimage of $R_{\mathbf{a}}^{0}(t)$ that belongs to $f_{\mathbf{a}}^{k-1}\left(P_{n+k}^{\mathbf{a}}\right)$, i.e. on $R_{\mathbf{a}}^{0}\left((d-1)^{k-1} t\right)$. Thus the ray $R_{\mathbf{a}_{1}}^{0}\left((d-1)^{k-1} t\right)$, as well as its iterated preimage $R_{\mathbf{a}_{1}}^{0}(t)$, is not stable. Then the point $z_{1}$ is, for the return map $f_{\mathbf{a}_{1}}^{k}$, a parabolic fixed point. Moreover its multiplier is 1 since the ray $R_{\mathbf{a}_{1}}^{0}(t)$ is fixed (by $f_{\mathbf{a}_{1}}^{k}$ ). Therefore, under the bijection $\chi_{\mathbf{a}_{0}}$ the parameter $\mathbf{a}_{1}$ corresponds to the cusp of $\mathbf{M}$, i.e. $\mathbf{a}_{1}=\chi_{\mathbf{a}_{0}}^{-1}(1 / 4)$.

Finally, the three rays $\mathcal{R}_{0}(t), \mathcal{R}_{\infty}(\zeta), \mathcal{R}_{\infty}\left(\zeta^{\prime}\right)$ converge to the same parameter $\mathbf{a}_{1}=\chi_{\mathbf{a}_{0}}^{-1}(1 / 4)$ of $\mathbf{M}_{\mathbf{a}_{0}}$, and the proof above shows that $\mathbf{M}_{\mathbf{a}_{0}} \subset \mathcal{P}_{n}$ is in the connected component of 
$\mathbf{C} \backslash\left(\overline{\mathcal{R}}_{0}\left(t_{n}\right) \cup \overline{\mathcal{R}}_{0}\left(t_{n}^{\prime}\right) \cup \overline{\mathcal{R}}_{\infty}\left(\zeta_{n}\right) \cup \overline{\mathcal{R}}_{\infty}\left(\zeta_{n}^{\prime}\right)\right)$ that contains $\mathbf{a}_{0}$. Therefore, $\mathbf{M}_{\mathbf{a}_{0}}$ is in the closure of the connected component of $\mathbf{C} \backslash\left(\overline{\mathcal{R}}_{\infty}(\zeta) \cup \overline{\mathcal{R}}_{\infty}\left(\zeta^{\prime}\right)\right)$ not containing $\mathcal{H}_{0}$. (Misiurewicz parameters in $\mathbf{M}_{\mathbf{a}_{0}}$ are accessible by external rays $\mathcal{R}_{\infty}(\theta)$ with $\theta \in\left(\zeta, \zeta^{\prime}\right)$.) Then the only possible intersection between $\mathbf{M}_{\mathbf{a}_{0}}$ and $\overline{\mathcal{H}}_{0}$ is the cusp $\mathbf{a}_{1}$. Therefore $\mathbf{a}_{1}=\mathbf{a}_{0}$.

The three rays $R_{\mathbf{a}_{0}}^{0}(t), R_{\mathbf{a}_{0}}^{\infty}(\zeta), R_{\mathbf{a}_{0}}^{\infty}\left(\zeta^{\prime}\right)$ converge to points in $\cap P_{n}^{\mathbf{a}_{0}}$, fixed by $f_{\mathbf{a}_{0}}^{k}$. But $K_{\mathbf{a}_{0}, k}=\bigcap P_{n}^{\mathbf{a}_{0}}$ contains only one fixed point with rotation number 1 called $\beta$. Thus the three rays land at the same point: $\beta$. They separate $K_{\mathbf{a}_{0}, k}$ from $B_{\mathbf{a}_{0}}$, i.e. $f_{\mathbf{a}_{0}}\left(-\mathbf{a}_{0}\right)$ from $B_{\mathbf{a}_{0}}$. Indeed, any eventually repelling periodic point in $K_{\mathbf{a}_{0}, k}$ (for instance $\beta^{\prime}$ the preimage of $\beta$ by $f_{\mathbf{a}_{0}}^{k}$ ) is accessible by an external ray whose angle is between $\zeta_{n}$ and $\zeta_{n}^{\prime}$, so at the limit between $\zeta$ and $\zeta^{\prime}$.

DEFINITION 4.7. - A parameter, a, is called parabolic (or of parabolic type) if $f_{\mathbf{a}}$ has a parabolic periodic point.

COROLlaRY 4.8. - Any $\mathbf{a}_{0} \in \partial \mathcal{H}_{0}$, for which $f_{\mathbf{a}_{0}}$ is renormalizable, is the cusp of a copy of $\mathbf{M}$. More precisely, the intersection $\mathbf{M}_{\mathbf{a}_{0}} \bigcap \partial \mathcal{H}_{0}$ reduces to $\left\{\mathbf{a}_{0}\right\}$ for $\mathbf{M}_{\mathbf{a}_{0}}=\bigcap \mathcal{P}_{n}\left(\mathbf{a}_{0}\right)$. Moreover $\mathbf{a}_{0}=\chi_{\mathbf{a}_{0}}^{-1}(1 / 4)$ where $\mathbf{M}=\chi \mathbf{a}_{0}\left(\mathbf{M}_{\mathbf{a}_{0}}\right)$, so $\mathbf{a}_{0}$ is parabolic.

Proof. - This follows from Proposition 4.6, its proof above and the use of symmetries.

COROLlARY 4.9. - The boundary of $\mathcal{H}_{0}$ is locally connected.

Proof. - It is locally connected at parameters $\mathbf{a} \in \partial \mathcal{H}_{0}$ which are not renormalizable (Lemma 4.5). For parameters $\mathbf{a}_{0} \in \partial \mathcal{H}_{0}$ which are renormalizable, we consider the sequence $\mathcal{Q}_{n}$ of subsets of $\partial \mathcal{H}_{0}$ defined by $\mathcal{Q}_{n}=\overline{\mathcal{P}_{n}} \cap \partial \mathcal{H}_{0}$. These subsets are connected neighbourhoods of $\mathbf{a}_{0}$ in $\partial \mathcal{H}_{0}$ (Lemma 4.3). Moreover they form a basis since $\left(\bigcap_{n \geqslant 0} \overline{\mathcal{P}_{n}} \cap \partial \mathcal{H}_{0}\right) \subset\left(\mathbf{M}_{\mathbf{a}_{0}} \cap \partial \mathcal{H}_{0}\right)=$ $\left\{\mathbf{a}_{0}\right\}$ by Corollary 4.8 .

The following lemma is used in Theorem 3.

Lemma 4.10. - Let $\mathbf{a} \in \mathcal{C}$. For any point $z \in \partial B_{\mathbf{a}}$, there are at most two external rays converging to $z$. Moreover, if $z$ is not eventually critical and if there are two external rays converging to $z$, then $z$ is (eventually) periodic; moreover the two rays define two connected components, each of them contains at least one critical point of $f_{\mathbf{a}}^{k}$ for some $k \geqslant 0$.

Proof. - Assume that $z$ is not eventually critical. Consider the closure of all the external rays converging to $z$ and let $V$ be a connected component of its complement. Assume to get a contradiction that there is no critical point of $f_{\mathbf{a}}^{k}$ in $V$ (for every $k \geq 0$ ). Then the iterates $f_{\mathbf{a}}^{k}$ restrict to homeomorphisms on $V$. This contradicts the fact that the map is doubling the angles on $B_{\mathbf{a}}(\infty)$ so that the image of $V$ by some $f_{\mathbf{a}}^{k}$ will contain all $B_{\mathbf{a}}(\infty)$ and $f_{\mathbf{a}}^{k+1}$ will no more be injective on $V$.

Assume now that there are at least three external rays converging to $z$. Let $V_{0}$ be the component containing the critical point 0 and (up to iterating) one component $V_{1}$ contains the first inverse image of $B_{\mathbf{a}}$. Note that this implies that $-\mathbf{a} \in V_{1}$. Take $V_{2}$ to be a third component. Since $V_{2}$ contains a critical point of $f_{\mathbf{a}}^{i}$ (for some $i \geqslant 0$ ) it is mapped by some iterate of $f_{\mathbf{a}}$, to $V_{1}$ (which contains $-\mathbf{a}$ and the preimage of $B_{\mathbf{a}}$ ). Indeed, all these sectors are mapped to sectors attached to $\partial B_{\mathbf{a}}$ as long as they do not contain a critical point. Therefore $z$ is a periodic point, of period say $k$. Then the fact that $V_{2}$ is mapped to $V_{1}$ contradicts the fact that $f_{\mathbf{a}}^{k}$ preserves the cyclic order of the rays landing at $z$ since there is a finite number of external rays landing at $z$ (see for instance [16]).

If a critical point $c$ is on the boundary of $B_{\mathbf{a}}$, by the previous description it is not possible that two external rays converge to $f(c)$. Therefore, $f(c)$ is the landing point of exactly one external ray and $c$ of exactly 2 and so for every $z$ which is eventually critical. 
Now we can describe more precisely the boundary of $\partial \mathcal{H}_{0}$ :

THEOREM. - 3. Let $\mathbf{a} \in \partial \mathcal{H}_{0} \backslash \mathbf{R}^{-}$; there exists a unique parameter ray in $\mathcal{H}_{0}$ landing at $\mathbf{a}$, say $\mathcal{R}_{0}^{s}(t)$. The following dichotomy holds:

- there is a unique external parameter ray converging to $\mathbf{a}$. In this case $f_{\mathbf{a}}$ is not renormalizable so that a does not belong to a copy of $\mathbf{M}$. Moreover in the dynamical plane, the ray $R_{\mathbf{a}}^{0}(t)$ lands at the critical value $f_{\mathbf{a}}(-\mathbf{a}) \in \partial B_{\mathbf{a}}$ and there is a unique external ray converging to $f_{\mathbf{a}}(-\mathbf{a})$;

- there are exactly two external parameter rays converging to $\mathbf{a}$. In this case $\mathbf{a}$ is the cusp of a copy of M. Furthermore, in the dynamical plane, the ray $R_{\mathbf{a}}^{0}(t)$ lands at a parabolic point on $\partial B_{\mathbf{a}}$. The angle $t$ is necessarily periodic by multiplication by $d-1$.

Note that in the first case, the angle $t$ can be periodic by multiplication by $d-1$ in this case a is a Misiurewicz parameter. In Proposition 2.32, we give the exact conditions on $t \in \mathbf{Q}$ so that a is of parabolic or of Misiurewicz type. If $t \in \mathbf{R} \backslash \mathbf{Q}$ we are clearly in the first case.

Proof. - We do the proof in several steps.

Any parameter a of $\partial \mathcal{H}_{0} \backslash \mathbf{R}^{-}$is the landing point of a ray in $\mathcal{H}_{0}$.

We consider the fundamental domain $s(\mathcal{S})$ containing a. For $d>3$, the map $\Phi_{0}$ restricts to a homeomorphism $\check{\Phi}_{0}$ from $s(\mathcal{S}) \cap \mathcal{H}_{0}$ onto $\Delta_{d}$ (see Proposition 2.4). Since the boundary of $\mathcal{H}_{0}$ and therefore of $s(\mathcal{S}) \cap \mathcal{H}_{0}$ (Remark 2.17) is locally connected, the inverse map $\Psi_{0}=\check{\Phi}_{0}^{-1}$ extends continuously to the boundaries: $\bar{\Psi}_{0}: \overline{\Delta_{d}} \rightarrow \overline{s(\mathcal{S}) \cap \mathcal{H}_{0}}$. The analogue statement for $d=3$ gives a continuous extension $\bar{\Psi}_{0}$ from $\overline{\mathbf{D}} \backslash \mathbf{R}^{+}$into $s(\dot{\mathcal{S}}) \cap \overline{\mathcal{H}_{0}}$ (one can use a double covering argument to see this). Therefore the parameter a on the boundary of $\mathcal{H}_{0}$ is the limit of a ray $\mathcal{R}_{0}^{s}(t)$.

Now we suppose first that $f_{\mathbf{a}}$ is not renormalizable.

Note that a does not belong to a copy of $\mathbf{M}$ by the definition of "renormalizable".

In the dynamical plane, the sequence of puzzle pieces $\left(P_{n}^{\mathbf{a}}\right)$ shrinks to one point namely $f_{\mathbf{a}}(-\mathbf{a})$. Moreover, taking the subsequence $n_{i}$ such that $\overline{\mathcal{P}_{n_{i}+1}} \subset \mathcal{P}_{n_{i}}$, the existence of the homeomorphism between $\partial \mathcal{P}_{n_{i}+1}$ and $\partial P_{n_{i}+1}^{\mathbf{a}}$ preserving angles and potentials (Corollary 3.20) insures that the ray $R_{\mathbf{a}}^{0}(t)$ enters all the puzzle pieces $P_{n_{i}+1}^{\mathbf{a}}$ for $i \geqslant 0$. Thus $R_{\mathbf{a}}^{0}(t)$ converges to $f_{\mathbf{a}}(-\mathbf{a})$.

By Lemma 4.10, there is only one external ray landing at $z=f_{\mathbf{a}}(-\mathbf{a})$ since $-\mathbf{a} \in \partial B_{\mathbf{a}}$. Assume now that two external rays $\mathcal{R}_{\infty}(\xi), \mathcal{R}_{\infty}\left(\xi^{\prime}\right)$ land at $\mathbf{a}$. These two rays enter any parapuzzle piece $\mathcal{P}_{n}(\mathbf{a})$ so by the homeomorphism of Corollary 3.20 the rays $R_{\infty}^{\mathbf{a}}(\xi)$ and $R_{\infty}^{\mathbf{a}}\left(\xi^{\prime}\right)$ enter all the pieces $P_{n}^{\mathbf{a}}$. Since the intersection $\bigcap_{n \geqslant 0} P_{n}^{\mathbf{a}}$ reduces to $f_{\mathbf{a}}(-\mathbf{a})$, the rays both converge to the same point $z=f_{\mathbf{a}}(-\mathbf{a})$. But we have just seen that this is not possible.

Now we consider the second case of the dichotomy: $f_{\mathbf{a}}$ is renormalizable.

In this case Proposition 3.26 insures that $\mathbf{M}_{\mathbf{a}}=\cap \mathcal{P}_{n}(\mathbf{a})$ is a copy of $\mathbf{M}$ and $\mathbf{a}$ is the cusp $\chi^{-1}(1 / 4)$ where $\chi$ is the homeomorphism between $\mathbf{M}_{\mathbf{a}}$ and $\mathbf{M}$. There are two external rays $\mathcal{R}_{\infty}(\zeta), \mathcal{R}_{\infty}\left(\zeta^{\prime}\right)$ converging to a by Proposition 4.6 and in the dynamical plane the ray $R_{\mathbf{a}}^{0}(t)$ converges to a point $z \in \partial B_{\mathbf{a}}$ which is a parabolic periodic point. Hence the angle $t$ is periodic by multiplication by $d-1$.

To prove that there are only two external parameter rays converging to a we proceed by contradiction. Assume that there is a third ray $\xi$ converging to a. To fix the ideas assume that the cyclic order at $\infty$ is $\zeta^{\prime}, \xi, \zeta$. Then the Mandelbrot copy belongs to one connected component of the complement of $\mathcal{R}_{\infty}(\xi) \cup \mathcal{R}_{\infty}(\zeta) \cup \mathcal{R}_{\infty}\left(\zeta^{\prime}\right) \cup\{\mathbf{a}\}$, say the one containing the rays of angle between $\zeta^{\prime}$ and $\xi$. Since the ray $\mathcal{R}_{\infty}(\xi)$ enters every para-puzzle piece, the ray $R_{\mathbf{a}}^{\infty}(\xi)$ enters every puzzle piece $P_{n}^{\mathbf{a}}$ by the homeomorphism of Corollary 3.20. So the ray $R_{\mathbf{a}}^{\infty}(\xi)$

$4^{\text {e }}$ SÉRIE - TOME $40-2007-\mathrm{N}^{\circ} 6$ 


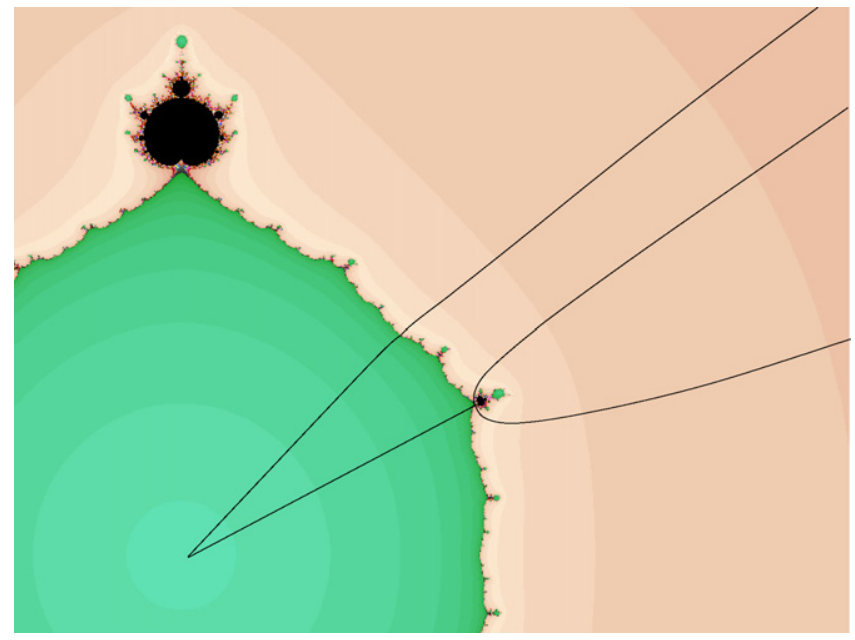

Fig. 9. Points on $\partial \mathcal{H}_{0}$ and rays converging to them.

converges to a point $z$ of $J\left(f_{\mathbf{a}}^{k}\right)$ (the Julia set of the renormalized map). There exist points in $J\left(f_{\mathbf{a}}^{k}\right)$ that are accessible by external ray of angle $\xi^{\prime} \in \mathbf{Q}$ between $\xi$ and $\zeta$ such that neither $\frac{t}{d}+\frac{\left\lfloor\frac{d-1}{2}\right\rfloor}{d}$ nor $\frac{t}{d}+\frac{\left\lfloor\frac{d-1}{2}\right\rfloor+1}{d}$ is periodic by multiplication by $d$. Then the ray $\mathcal{R}_{\infty}\left(\xi^{\prime}\right)$ lands at a Misiurewicz parameter by Proposition 2.31. This Misiurewicz parameter belongs to $\mathbf{M}_{\mathbf{a}}$ since the ray $\mathcal{R}_{\infty}\left(\xi^{\prime}\right)$ enters every para-puzzle piece $\mathcal{P}_{n}$ (by the homeomorphism of Corollary 3.20). But this contradicts the fact that $\mathbf{M}_{\mathbf{a}}$ belongs to the connected component containing the rays of angle between $\zeta^{\prime}$ and $\xi$.

Remark 4.11. - The fact that $t$ is $k$-periodic by multiplication by $d-1$ does not imply that $f_{\mathbf{a}}$ is renormalizable.

Proof. - It is possible that the sequence $f_{\mathbf{a}}^{i}\left(P_{n+k}^{\mathbf{a}}\right)$ avoids the critical point since there are other preimages of $f_{\mathbf{a}}(-\mathbf{a})$ on $\partial B_{\mathbf{a}}$ in degree $d>3$. See Proposition 2.32.

DEFINITION 4.12. - We define the wake $\mathcal{W}\left(\mathbf{a}_{0}\right)$ of any point $\mathbf{a}_{0} \in \partial \mathcal{H}_{0} \cap \mathcal{S}$ as follows.

If $f_{\mathbf{a}_{0}}$ is not renormalizable let us take $\mathcal{W}\left(\mathbf{a}_{0}\right)=\emptyset$; else let $\mathcal{W}\left(\mathbf{a}_{0}\right)$ be the connected component of

$$
\mathbf{C} \backslash\left(\overline{\mathcal{R}}_{\infty}\left(\zeta_{0}\right) \cup \overline{\mathcal{R}}_{\infty}\left(\zeta_{0}^{\prime}\right)\right) \text { containing } \mathbf{M}_{\mathbf{a}_{0}} \backslash\left\{\mathbf{a}_{0}\right\}
$$

where $\zeta_{0}, \zeta_{0}^{\prime}$ are periodic angles (by multiplication by $d$ ) such that the rays $\mathcal{R}_{\infty}\left(\zeta_{0}\right)$ and $\mathcal{R}_{\infty}\left(\zeta_{0}^{\prime}\right)$ converge to $\mathbf{a}_{0}$. For parameters not in $\mathcal{S}$ we use the symmetries to define the wake.

Remark 4.13. - By Theorem 3 there are at most two rays converging to a parameter $\mathbf{a} \in \partial \mathcal{H}_{0}$ (those defined in Proposition 4.6) so that the wake is well defined. Moreover since $\mathbf{a}$ is the landing point of a ray $\mathcal{R}_{0}^{s}(t)$, we can also call $\mathcal{W}^{s}(t)$ the wake $\mathcal{W}(\mathbf{a})$. Note that the wake of a parameter $\mathbf{a} \in \mathcal{S}$ is not necessarily contained in $\mathcal{S}$.

LEMMA 4.14. - For any parameter a in $\mathcal{W}\left(\mathbf{a}_{0}\right)$, the rays $R_{\mathbf{a}}^{\infty}\left(\zeta_{0}\right), R_{\mathbf{a}}^{\infty}\left(\zeta_{0}^{\prime}\right)$ and $R_{\mathbf{a}}^{0}\left(t_{0}\right)$ converge to the same point which is repelling of period $k$ (the period of $\mathbf{M}_{\mathbf{a}_{0}}$ ), where $\zeta_{0}, \zeta_{0}^{\prime}$ define the wake $\mathcal{W}\left(a_{0}\right)$ and $\mathcal{R}_{0}^{s}\left(t_{0}\right)$ is landing at $\mathbf{a}_{0}$. For $\mathbf{a}=\mathbf{a}_{0}$ these three dynamical rays also land at a common point, which is a k-periodic parabolic point. Moreover, for $\mathbf{a} \in \mathcal{W}\left(\mathbf{a}_{0}\right) \cup$ $\left\{\mathbf{a}_{0}\right\}$, the critical value is in the corresponding dynamical wake: the connected component of $\mathbf{C} \backslash\left(\bar{R}_{\mathbf{a}}^{\infty}\left(\zeta_{0}\right) \cup \bar{R}_{\mathbf{a}}^{\infty}\left(\zeta_{0}^{\prime}\right)\right)$ which does not contain $B_{\mathbf{a}}$. 


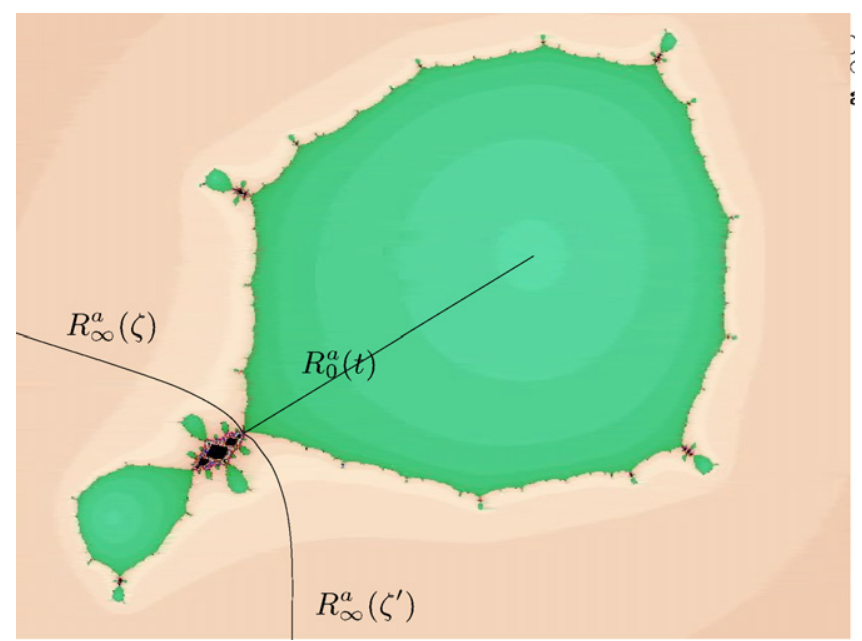

Fig. 10. Illustration of Lemma 4.14, $\mathbf{a} \in \mathbf{M}_{\mathbf{a}_{0}}$ of Fig. 8 .

Proof. - Note first that, for every parameter $\mathbf{a} \in \mathbf{M}_{\mathbf{a}_{0}}$, the three rays $R_{\mathbf{a}}^{\infty}\left(\zeta_{0}\right), R_{\mathbf{a}}^{\infty}\left(\zeta_{0}^{\prime}\right)$ and $R_{\mathbf{a}}^{0}\left(t_{0}\right)$ converge to the same $k$-periodic point. For $\mathbf{a}=\mathbf{a}_{0}$, this follows from Proposition 4.6. Then it is easy to check that all the arguments of Proposition 4.6 go through for the parameters $\mathbf{a}$ in $\mathbf{M}_{\mathbf{a}_{0}}$. Indeed, the boundary of the puzzle pieces $\partial P_{n}^{\mathbf{a}}$ and $\partial P_{n}^{\mathbf{a}_{0}}$ are identified through the holomorphic motion defined on the neighbourhood $\mathcal{P}_{n-1}$ of $\mathbf{M}_{\mathbf{a}_{0}}$.

After this remark, the proof goes exactly as point 3 of Lemma 3.8 and Corollary 3.10, so we give here just the steps of the argumentation. We consider the set $\Omega$ of parameters a such that $R_{\mathbf{a}}^{\infty}\left(\zeta_{0}\right), R_{\mathbf{a}}^{\infty}\left(\zeta_{0}^{\prime}\right)$ and $R_{\mathbf{a}}^{0}\left(t_{0}\right)$ converge to the same point which is a repelling periodic point of period $k$. Then $\Omega$ is open and non-empty. Its boundary is included in $P P_{k} \cup \bigcup_{i \geqslant 0}\left(\mathcal{R}_{0}((d-\right.$ $\left.1)^{i} t_{1}\right) \cup \bigcup \mathcal{R}_{\infty}\left(d^{i} \zeta_{0}\right) \cup \bigcup \mathcal{R}_{\infty}\left(d^{i} \zeta_{0}^{\prime}\right)$ ) (see Definition 2.11), where $P P_{k}$ is the set of parameters a such that $f_{\mathbf{a}}$ has a parabolic point of period $k$ and multiplier 1 . We claim that, in the region $\mathcal{W}\left(\mathbf{a}_{0}\right)$, there are no parameter rays of $\bigcup \mathcal{R}_{\infty}\left(d^{i} \zeta_{0}\right), \bigcup \mathcal{R}_{\infty}\left(d^{i} \zeta_{0}^{\prime}\right)$ and $\mathcal{R}_{0}\left((d-1)^{i} t_{0}\right)$ with $i \geqslant 0$. For this we look first in the dynamical plane of $f_{\mathbf{a}_{0}}$. The angles $\zeta_{0}, \zeta_{0}^{\prime}$ are the limits of the sequences $\left(\zeta_{n}\right)_{n \geqslant 2},\left(\zeta_{n}^{\prime}\right)_{n \geqslant 2}$ defined as follows: the two internal rays $R_{\mathbf{a}_{0}}^{0}\left(t_{n}\right), R_{\mathbf{a}_{0}}^{0}\left(t_{n}^{\prime}\right)$ of $\partial P_{n}^{\mathbf{a}_{0}}$ converge to points $z_{n}, z_{n}^{\prime}$ to which are attached the external rays $R_{\mathbf{a}_{0}}^{\infty}\left(\zeta_{n}\right), R_{\mathbf{a}_{0}}^{\infty}\left(\zeta_{n}^{\prime}\right)$ of $\partial P_{n}^{\mathbf{a}_{0}}$. There is no iterate of $R_{\mathbf{a}_{0}}^{\infty}\left(\zeta_{n}\right), R_{\mathbf{a}_{0}}^{\infty}\left(\zeta_{n}^{\prime}\right)$ in $Q_{n}$, the connected component containing $f_{\mathbf{a}_{0}}\left(-\mathbf{a}_{0}\right)$ of

$$
\mathbf{C} \backslash\left(\overline{R_{\mathbf{a}_{0}}^{0}\left(t_{n}\right)} \cup \overline{R_{\mathbf{a}_{0}}^{0}\left(t_{n}^{\prime}\right)} \cup \overline{R_{\mathbf{a}_{0}}^{\infty}\left(\zeta_{n}\right)} \cup \overline{R_{\mathbf{a}_{0}}^{\infty}\left(\zeta_{n}^{\prime}\right)}\right) .
$$

Otherwise, such an iterate would be attached to an internal ray of some $\partial P_{j}^{\mathbf{a}_{0}}$ for $j \leqslant n$, with angle in $\left(t_{n}, t_{n}^{\prime}\right)$. But this is not possible since $\partial P_{n}^{\mathbf{a}_{0}}$ contains only two rays in $B_{\mathbf{a}_{0}}$, they are in $\partial Q_{n}$, and since $P_{n}^{\mathbf{a}_{0}} \subset P_{j}^{\mathbf{a}_{1}}$. Therefore, there is no element of $d^{i} \zeta_{0}, d^{i} \zeta_{0}^{\prime}$ in the segment $\left(\zeta_{n}, \zeta_{n}^{\prime}\right)$ "defining" the region $Q_{n}$, and so neither in the limit interval $\left(\zeta_{0}, \zeta_{0}^{\prime}\right)$. Thus there are no rays of $\bigcup \mathcal{R}_{\infty}\left(d^{i} \zeta_{0}\right), \bigcup \mathcal{R}_{\infty}\left(d^{i} \zeta_{0}^{\prime}\right), i \geqslant 0$ in $\mathcal{W}\left(\mathbf{a}_{0}\right)$. On the other hand, there is no point of $P P_{k}$ in $\mathcal{W}\left(\mathbf{a}_{0}\right)$ : otherwise this would contradict the maximum principle for the multiplier of the landing point of $R_{\mathbf{a}}^{0}\left(t_{0}\right)$ (as in Lemma 3.8). Therefore, $\mathcal{W}\left(\mathbf{a}_{0}\right) \subset \Omega$.

\subsection{Local connectivity of $\partial \mathcal{U}$ for a component $\mathcal{U}$ of $\mathcal{H} \backslash \mathcal{H}_{0}$}

PROPOSITION 4.15. - Let $\mathcal{U}$ be a connected component of $\mathcal{H} \backslash \mathcal{H}_{0}$. Let $\mathbf{a}_{0} \in \partial \mathcal{U} \cap \mathcal{S}$ be such that $f_{\mathbf{a}_{0}}$ is renormalizable and denote by $\chi_{\mathbf{a}_{0}}$ the homeomorphism between $\mathbf{M}_{\mathbf{a}_{0}}$ and $\mathbf{M}$ where $\mathbf{M}_{\mathbf{a}_{0}}=\cap \mathcal{P}_{n}\left(\mathbf{a}_{0}\right)$ (see Proposition 4.6). Then:

$4^{\mathrm{e}}$ SÉRIE - TOME $40-2007-\mathrm{N}^{\circ} 6$ 


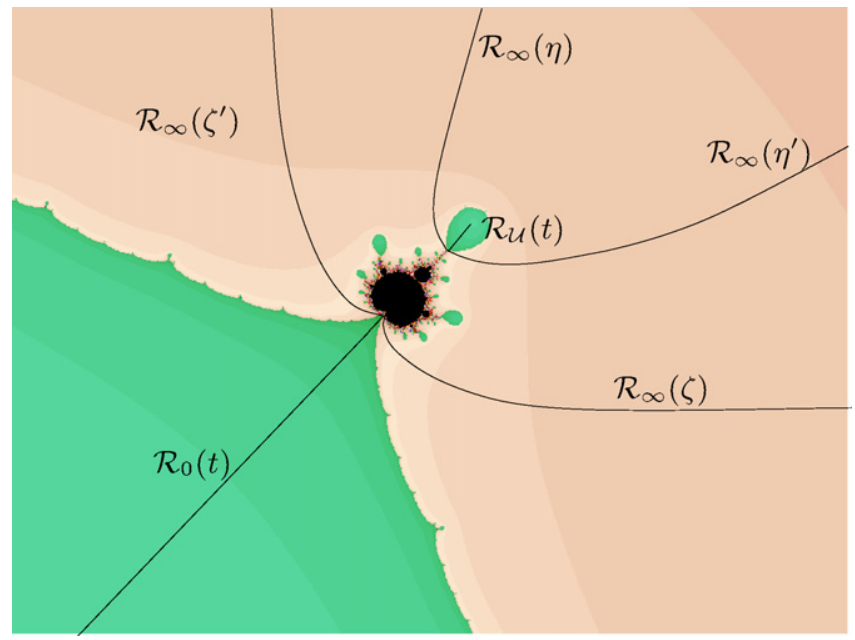

Fig. 11. Intersection of $\mathcal{U}$ and the copy $\mathbf{M}_{0}$ of $\mathbf{M}$.

$-\partial \mathcal{U} \cap \mathbf{M}_{\mathbf{a}_{0}}=\left\{\mathbf{a}_{0}\right\} ;$

- $\mathbf{M}_{\mathbf{a}_{0}} \cap \partial \mathcal{H}_{0}=\chi \chi_{\mathbf{a}_{0}}^{-1}(1 / 4):=\mathbf{a}_{1}$ the cusp of $\mathbf{M}_{\mathbf{a}_{0}}$;

$-\mathcal{U} \subset \mathcal{W}\left(\mathbf{a}_{1}\right)$ the wake of $\mathbf{a}_{1}$;

- $\mathbf{a}_{0}$ is the landing point of three rays $\mathcal{R}_{\mathcal{U}}\left(t_{1}\right), \mathcal{R}_{\infty}(\eta)$ and $\mathcal{R}_{\infty}\left(\eta^{\prime}\right)$ where $d^{i} \eta=\zeta_{1}, d^{i} \eta^{\prime}=\zeta_{1}^{\prime}$ with $t_{1}$ and $\zeta_{1}, \zeta_{1}^{\prime} k$-periodic by multiplication by $d-1$, and by $d$ respectively. Here $i$ is the depth of $\mathcal{U}$, i.e., $\mathcal{U} \subset \mathcal{H}_{i} \backslash \mathcal{H}_{i-1}$, and $t_{1}, \zeta_{1}, \zeta_{1}^{\prime}$ are associated to $\mathbf{a}_{1}$ by Proposition 4.6;

- the curve $\overline{\mathcal{R}}_{\infty}(\eta) \cup \overline{\mathcal{R}}_{\infty}\left(\eta^{\prime}\right)$ separates $\mathcal{U}$ from $\mathbf{M}_{\mathbf{a}_{0}}$.

Proof. - We will not prove the items in the order they appear. The proof is very similar to that of Proposition 4.6. Using Lemma 2.22, we can define in $\mathcal{P}_{n}=\mathcal{P}_{n}\left(\mathbf{a}_{0}\right)$ a holomorphic function $r(\mathbf{a})$ which coincides, for $\mathbf{a} \in \mathcal{U}$, with the center of $U(\mathbf{a})$, the connected component of $\widetilde{B}_{\text {a }}$ containing the critical value. Since $\mathcal{P}_{n}$ intersects $\mathcal{U}, \partial \mathcal{P}_{n}$ contains two internal rays $\mathcal{R}_{\mathcal{U}}\left(\tau_{n}\right), \mathcal{R}_{\mathcal{U}}\left(\tau_{n}^{\prime}\right)$ (Lemma 4.2) with landing points $u_{n}, u_{n}^{\prime}$ respectively, but also external rays $\mathcal{R}_{\infty}\left(\eta_{n}\right), \mathcal{R}_{\infty}\left(\eta_{n}^{\prime}\right)$ landing at $u_{n}, u_{n}^{\prime}$ respectively. Using the homeomorphism of Corollary 3.20, the boundary $\partial P_{n}^{\mathbf{a}_{0}}$ contains the part in $\mathcal{X}_{n}$ of the rays $R_{\mathbf{a}_{0}}^{r\left(\mathbf{a}_{0}\right)}\left(\tau_{n}\right), R_{\mathbf{a}_{0}}^{r\left(\mathbf{a}_{0}\right)}\left(\tau_{n}^{\prime}\right)$ and $R_{\mathbf{a}_{0}}^{\infty}\left(\eta_{n}\right)$, $R_{\mathbf{a}_{0}}^{\infty}\left(\eta_{n}^{\prime}\right)$ (Corollary 2.23), with common landing points, say $z_{n}, z_{n}^{\prime}$ respectively, at least for $n$ in the subsequence $\left(n_{i}\right)_{i \geq 0}$ given in Proposition 3.22.

1. We prove first that $\partial \mathcal{P}_{n} \cap \mathcal{H}_{0} \neq \emptyset$ and that $\mathbf{M}_{\mathbf{a}_{0}} \cap \partial \mathcal{H}_{0}=\chi_{\mathbf{a}_{0}}^{-1}(1 / 4):=\mathbf{a}_{1}$ :

Since for every $m \geqslant 0, P_{m}^{\mathbf{a}_{0}}$ intersects $U(\mathbf{a})$, which is of depth $i$, the image $f_{\mathbf{a}_{0}}^{i}\left(P_{m}^{\mathbf{a}_{0}}\right)$ intersects $B_{\mathbf{a}_{0}}$. Then, for $i \leqslant k j \leqslant m$, the image $f_{\mathbf{a}_{0}}^{k j}\left(P_{m}^{\mathbf{a}_{0}}\right)$ which is the puzzle piece $P_{m-k j}^{\mathbf{a}_{0}}$ containing the critical value, intersects $B_{\mathbf{a}_{0}}$. Thus, $P_{n}^{\mathbf{a}_{0}} \cap B_{\mathbf{a}_{0}} \neq \emptyset$ for any $n$. By the homeomorphism of Corollary 3.20, we deduce that $\mathcal{P}_{n} \cap \mathcal{H}_{0} \neq \emptyset$. So there is some point $\mathbf{a}_{1}$ in the intersection $\bigcap_{n \geqslant 0} \mathcal{P}_{n} \cap \overline{\mathcal{H}}_{0}=\mathbf{M}_{\mathbf{a}_{0}} \cap \overline{\mathcal{H}}_{0}$. Applying Proposition 4.6 (or Corollary 4.8) to this point $\mathbf{a}_{1}$, we deduce that $\mathbf{M}_{\mathbf{a}_{0}} \cap \partial \mathcal{H}_{0}$ is reduced to $\mathbf{a}_{1}$ and is the cusp of $\chi_{\mathbf{a}_{0}}^{-1}(1 / 4)$ of $\mathbf{M}_{\mathbf{a}_{0}}$ since $\mathbf{M}_{\mathbf{a}_{1}}=\mathbf{M}_{\mathbf{a}_{0}}$. Indeed, $\mathbf{a}_{1} \in \mathbf{M}_{\mathbf{a}_{0}}=\cap \mathcal{P}_{n}$, the pieces $\mathcal{P}_{n}\left(\mathbf{a}_{0}\right)$ and $\mathcal{P}_{n}\left(\mathbf{a}_{1}\right)$ coincide.

2. We prove here that $\mathcal{P}_{n}$ contains in its boundary the part in $\mathcal{X}_{n}$ of the rays $\mathcal{R}_{0}\left(\tau_{n+i}\right)$, $\mathcal{R}_{0}\left(\tau_{n+i}^{\prime}\right), \mathcal{R}_{\infty}\left(\zeta_{n+i}\right), \mathcal{R}_{\infty}\left(\zeta_{n+i}^{\prime}\right)$ with $\zeta_{n}=d^{i} \eta_{n}$ and $\zeta_{n}^{\prime}=d^{i} \eta_{n}^{\prime}$, at least for infinitely many $n \in \mathbf{N}$ :

We prove it for the dynamical puzzle piece $P_{n}^{\mathbf{a}_{0}}$ and then use the homeomorphism of Corollary 3.20. We have seen in point 1 above that the puzzle piece $P_{n+i}^{\mathbf{a}_{0}}$ contains in its boundary 
the rays $R_{\mathbf{a}_{0}}^{r\left(\mathbf{a}_{0}\right)}\left(\tau_{n+i}\right), R_{\mathbf{a}_{0}}^{r\left(\mathbf{a}_{0}\right)}\left(\tau_{n+i}^{\prime}\right)$ and also $R_{\mathbf{a}_{0}}^{0}\left(t_{n+i}\right), R_{\mathbf{a}_{0}}^{0}\left(t_{n+i}^{\prime}\right)$. Since $f_{\mathbf{a}_{0}}^{i}\left(P_{n+i}^{\mathbf{a}_{0}}\right)$ is the piece $P_{n}^{\mathbf{a}_{0}}\left(f_{\mathbf{a}_{0}}^{i}\left(f_{\mathbf{a}_{0}}\left(-\mathbf{a}_{0}\right)\right)\right)$, it contains only two rays of $B_{\mathbf{a}_{0}}$ in its boundary, so that the rays $R_{\mathbf{a}_{0}}^{r\left(\mathbf{a}_{0}\right)}\left(\tau_{n+i}\right)$ and $R_{\mathbf{a}_{0}}^{0}\left(t_{n+i}^{\prime}\right)$ have the same image by $f_{\mathbf{a}_{0}}^{i}$. In particular, $(d-1)^{i} t_{n+i}^{\prime}=\tau_{n+i}=$ $t_{n}^{\prime}$. Therefore the puzzle piece $f_{\mathbf{a}_{0}}^{i-1}\left(P_{n+i}^{\mathbf{a}_{0}}\right)$ contains the critical point $-\mathbf{a}_{0}$ since it is simply connected and $f_{\mathbf{a}_{0}}$ maps its boundary with degree two on its image. Thus $f_{\mathbf{a}_{0}}^{i}\left(P_{n+i}^{\mathbf{a}_{0}}\right)=P_{n}^{\mathbf{a}_{0}}$ so that $i$ is a multiple of $k$. The piece $P_{n}^{\mathbf{a}_{0}}$ contains in its boundary the rays $R_{\mathbf{a}_{0}}^{0}\left(\tau_{n+i}\right), R_{\mathbf{a}_{0}}^{0}\left(\tau_{n+i}^{\prime}\right)$, with end points $z_{n+i}, z_{n+i}^{\prime}$ respectively, to which converge the external rays $R_{\mathbf{a}_{0}}^{\infty}\left(\zeta_{n+i}\right)$, $R_{\mathbf{a}_{0}}^{\infty}\left(\zeta_{n+i}^{\prime}\right)$ with $\zeta_{n+i}=d^{i} \eta_{n+i}, \zeta_{n+i}^{\prime}=d^{i} \eta_{n+i}^{\prime}$.

Using the homeomorphism of Corollary 3.20 we deduce that the para-puzzle piece $\mathcal{P}_{n}$ contains in its boundary the rays $\mathcal{R}_{0}\left(\tau_{n+i}\right), \mathcal{R}_{\infty}\left(\zeta_{n+i}\right)$ landing at a common parameter $\mathbf{a}_{n+i}$ and $\mathcal{R}_{0}\left(\tau_{n+i}^{\prime}\right), \mathcal{R}_{\infty}\left(\zeta_{n+i}^{\prime}\right)$ landing at some other parameter $\mathbf{a}_{n+i}^{\prime}$, at least for $n$ in the subsequence $\left(n_{j}\right)_{j \geqslant 0}$ defined in Proposition 3.22.

3. We prove now that $\mathcal{U} \subset \mathcal{W}\left(\mathbf{a}_{1}\right)$ :

The pieces $\mathcal{P}_{n}\left(\mathbf{a}_{0}\right)$ and $\mathcal{P}_{n}\left(\mathbf{a}_{1}\right)$ coincide. As in Proposition 4.6 applied to $\mathbf{a}_{1}$, the sequences of angles $\left(\tau_{n}\right),\left(\tau_{n}^{\prime}\right)$ admit a common limit $\tau$ which is, by point 2 above, equal to the common limit $t_{1}$ of the sequences $\left(t_{n}\right)_{n \geqslant 2},\left(t_{n}^{\prime}\right)_{n \geqslant 2}$. The monotone sequences $\left(\zeta_{n}\right)_{n \geqslant 2},\left(\zeta_{n}^{\prime}\right)_{n \geqslant 2}$ converge to limits $\zeta_{1}, \zeta_{1}^{\prime}$ respectively. By Proposition 4.6, the angle $\tau$ is $k$-periodic by multiplication by $d-1$, the angles $\zeta_{1}, \zeta_{1}^{\prime}$ are $k$-periodic by multiplication by $d$ and the rays $\mathcal{R}_{0}\left(t_{1}\right), \mathcal{R}_{\infty}\left(\zeta_{1}\right), \mathcal{R}_{\infty}\left(\zeta_{1}^{\prime}\right)$ land at $\mathbf{a}_{1}=\chi_{\mathbf{a}_{0}}^{-1}(1 / 4)$. The curve $\overline{\mathcal{R}_{\infty}\left(\zeta_{1}\right)} \cup \overline{\mathcal{R}_{\infty}\left(\zeta_{1}^{\prime}\right)}$ bounds $\mathcal{W}\left(\mathbf{a}_{1}\right)$.

Let $Q_{n}$ be the connected component containing $\mathbf{a}_{0}$ of

$$
\mathbf{C} \backslash\left(\overline{\mathcal{R}_{0}\left(\tau_{n+i}\right)} \cup \overline{\mathcal{R}_{0}\left(\tau_{n+i}^{\prime}\right)} \cup \overline{\mathcal{R}_{\infty}\left(\zeta_{n+i}\right)} \cup \overline{\mathcal{R}_{\infty}\left(\zeta_{n+i}^{\prime}\right)}\right) .
$$

The para-puzzle piece $\mathcal{P}_{n}$ is contained in $Q_{n}$ and $\mathcal{R}_{\mathcal{U}}\left(\tau_{n}\right)$ is in the boundary of $\mathcal{P}_{n}$. Thus $\mathcal{U}$ is included in $Q_{n}$ since $\mathcal{U} \cap \partial Q_{n}=\emptyset$. Thus the component $\mathcal{U}$ is totally included in $\mathcal{W}\left(\mathbf{a}_{1}\right)$.

4. Finally, we prove that $\mathcal{R}_{\mathcal{U}}\left(t_{1}\right), \mathcal{R}_{\infty}(\eta), \mathcal{R}_{\infty}\left(\eta^{\prime}\right)$ land at the same parameter, which is $\mathbf{a}_{0}$, and that the curve $\gamma=\overline{\mathcal{R}_{\infty}(\eta)} \cup \overline{\mathcal{R}_{\infty}\left(\eta^{\prime}\right)}$ separates $\mathcal{U}$ from $M_{\mathbf{a}_{0}}$ :

Since $t_{1}, \eta, \eta^{\prime}$ are rational $\left(d^{i} \eta=\zeta_{1}\right.$ and $\left.d^{i} \eta^{\prime}=\zeta_{1}^{\prime}\right)$ the rays $\mathcal{R}_{\mathcal{U}}\left(t_{1}\right), \mathcal{R}_{\infty}(\eta), \mathcal{R}_{\infty}\left(\eta^{\prime}\right)$ converge to parameters $\mathbf{a}_{t_{1}}, \mathbf{a}_{\eta}, \mathbf{a}_{\eta^{\prime}}$ respectively which are either parabolic or Misiurewicz parameters after Lemma 2.24.

If $\mathbf{a}_{t_{1}}$ is a Misiurewicz parameter, the ray $R_{\mathbf{a}_{t_{1}}}^{r\left(\mathbf{a}_{t_{1}}\right)}\left(t_{1}\right)$ converges to $f_{\mathbf{a}_{t_{1}}}\left(-\mathbf{a}_{t_{1}}\right)$ (Lemma 2.24). Moreover, the rays $R_{\mathbf{a}_{t_{1}}}^{0}\left(t_{1}\right), R_{\mathbf{a}_{t_{1}}}^{\infty}\left(\zeta_{1}\right)$ and $R_{\mathbf{a}_{t_{1}}}^{\infty}\left(\zeta_{1}^{\prime}\right)$ land at the same point which is repelling (Lemma 4.14). Indeed, $\mathbf{a}_{t_{1}}$ is in $\mathcal{W}\left(\mathbf{a}_{1}\right)$ since $\overline{\mathcal{R}_{\mathcal{U}}\left(t_{1}\right)} \subset \mathcal{W}\left(\mathbf{a}_{1}\right) \cup\left\{\mathbf{a}_{1}\right\}$ but $\mathbf{a}_{t_{1}} \neq \mathbf{a}_{1}$ since $\mathbf{a}_{1}$ is a parabolic parameter. Pulling back along the critical orbit we obtain that $R_{\mathbf{a}_{t_{1}}}^{r\left(\mathbf{a}_{t_{1}}\right)}\left(t_{1}\right), R_{\mathbf{a}_{t_{1}}}^{\infty}(\eta)$ and $R_{\mathbf{a}_{t_{1}}}^{\infty}\left(\eta^{\prime}\right)$ land at the same point, i.e., at $f_{\mathbf{a}_{t_{1}}}\left(-\mathbf{a}_{t_{1}}\right)$. Therefore, by Lemma 2.26 the rays $\mathcal{R}_{\mathcal{U}}\left(t_{1}\right)$, $\mathcal{R}_{\infty}(\eta), \mathcal{R}_{\infty}\left(\eta^{\prime}\right)$ land at the same parameter $\mathbf{a}_{t_{1}}$. The proof is exactly the same in case $\mathbf{a}_{\eta}$, or $\mathbf{a}_{\eta^{\prime}}$, is of Misiurewicz type.

Assume now that every parameter $\mathbf{a} \in\left\{\mathbf{a}_{t_{1}}, \mathbf{a}_{\eta}, \mathbf{a}_{\eta^{\prime}}\right\}$ is parabolic. Then, the landing point of $R_{\mathrm{a}}^{0}\left(t_{1}\right)$ is a parabolic periodic point. Thus, the map $f_{\mathrm{a}}^{k}$ possesses a parabolic fixed point of multiplier 1 . Then, the only possibility for $\mathbf{a} \in \mathbf{M}_{\mathbf{a}_{0}}$ is to be the cusp of $\mathbf{M}_{\mathbf{a}_{0}}$, i.e. $\mathbf{a}=\chi_{\mathbf{a}_{0}}^{-1}(1 / 4)$ so $\mathbf{a}_{t_{1}}=\mathbf{a}_{\eta}=\mathbf{a}_{\eta^{\prime}}$.

In both cases, the curve $\gamma=\mathcal{R}_{\infty}(\eta) \cup \mathcal{R}_{\infty}\left(\eta^{\prime}\right) \cup\left\{\mathbf{a}_{t_{1}}\right\}$ separates the plane into two components. Let $V$ denote the one containing $\mathcal{U}$ (since $\mathcal{U} \cap \gamma=\emptyset$ ). The para-puzzle piece $\mathcal{P}_{n}$ intersects $V$ and $\mathcal{U}$. Using Lemma 4.3 one can see that for any ray $\mathcal{R}_{\infty}(\xi)$ in $V \cap \mathcal{P}_{n}$, the angle $\xi$ is either in $\left(\eta^{\prime}, \eta_{n}^{\prime}\right)$ or in $\left(\eta_{n}, \eta\right)$. Assume (to get a contradiction) that $\mathbf{M}_{\mathbf{a}_{0}}$ intersects $V$. Then let $\mathbf{a}$ be a Misiurewicz parameter in the intersection $\mathbf{M}_{\mathbf{a}_{0}} \cap V$. It is the landing point of an external ray $\mathcal{R}_{\infty}(\xi)$. This ray belongs to $V$ but also enters every $\mathcal{P}_{n}$ since it converges to $\mathbf{a} \in \mathbf{M}_{\mathbf{a}_{0}}$. Hence $\xi$ is either in $\left(\eta^{\prime}, \eta_{n}^{\prime}\right)$ or in $\left(\eta_{n}, \eta\right)$, so $\xi=\eta$ or $\xi=\eta^{\prime}$. Then $\mathbf{a}=\mathbf{a}_{t_{1}}$ but this contradicts

$4^{\text {e }}$ SÉRIE - TOME $40-2007-\mathrm{N}^{\circ} 6$ 
the fact that $\mathbf{a} \in V$ (since $\mathbf{a}_{t_{1}} \in \partial V$ ). Therefore, the curve $\gamma$ separates $\mathbf{M}_{\mathbf{a}_{0}}$ from $\mathcal{U}$ and the unique possible intersection between $\overline{\mathcal{U}}$ and $\mathbf{M}_{\mathbf{a}_{0}}$ is $\mathbf{a}_{t_{1}}$, so that $\mathbf{a}_{0}=\mathbf{a}_{t_{1}}$ since $\mathbf{a}_{0} \in \mathbf{M}_{\mathbf{a}_{0}}$ and $\mathbf{a}_{0} \in \overline{\mathcal{U}}$.

We will see in Theorem 4 that $\mathbf{a}_{0}$ is always a Misiurewicz parameter.

COROLLARY 4.16. - If $\mathcal{U}$ is a connected component of $\mathcal{H} \backslash \mathcal{H}_{0}$, there exists at most one parameter $\mathbf{a}$ on $\partial \mathcal{U}$ such that $f_{\mathbf{a}}$ is renormalizable. If it exists it is the parameter characterized in Proposition 4.15.

Proof. - Let a be a parameter on $\partial \mathcal{U}$ such that $f_{\mathbf{a}}$ is renormalizable. By Proposition 4.15 $\mathbf{M}_{\mathbf{a}}=\bigcap \mathcal{P}_{n}(\mathbf{a})$ intersects $\overline{\mathcal{H}}_{0}$ and $\overline{\mathcal{U}}$. So if there is another point $\mathbf{a}^{\prime}$ like this, one can find a loop in $\overline{\mathcal{H}}_{0} \cup \overline{\mathcal{U}} \cup \mathbf{M}_{\mathbf{a}} \cup \mathbf{M}_{\mathbf{a}^{\prime}}$ surrounding points of $\mathcal{H}_{\infty}$ and this contradicts the fact that $\mathcal{H}_{\infty} \cup\{\infty\}$ is simply connected.

\section{COROLlary 4.17. - The boundary of $\mathcal{U}$ is locally connected.}

Proof. - Let $\mathbf{a}_{0} \in \partial \mathcal{U}$. If $f_{\mathbf{a}_{0}}$ is not renormalizable the result follows from Lemma 4.5. If $f_{\mathbf{a}_{0}}$ is renormalizable, we are in the situation of Proposition 4.15. The sequence $\overline{\mathcal{P}_{n}} \cap \partial \mathcal{U}$ of connected neighbourhoods of $\mathbf{a}_{0}$ (Lemma 4.3) is a basis since $\left(\bigcap_{n \geqslant 0} \overline{\mathcal{P}_{n}} \cap \partial \mathcal{U}\right) \subset\left(\mathbf{M}_{\mathbf{a}_{0}} \cap \partial \mathcal{U}\right)=\left\{\mathbf{a}_{0}\right\}$.

THEOREM 2. - For every connected component $\mathcal{U}$ of $\mathcal{H}$, the boundary $\partial \mathcal{U}$ is a Jordan curve.

Proof. - Every component $\mathcal{U}$ of $\mathcal{H}$ is simply connected (Lemma 1.12) and its boundary is locally connected (Corollary 4.17). Therefore any conformal map $\Psi: \mathbf{D} \rightarrow \mathcal{U}$ extends continuously to a map $\bar{\Psi}: \overline{\mathbf{D}} \rightarrow \overline{\mathcal{U}}$ by Caratheodory's Theorem. Thus the boundary $\partial \mathcal{U}$ is the curve: $\bar{\Psi}\left(\mathbf{S}^{1}\right)$. We prove that it is a Jordan curve by contradiction. If $\bar{\Psi}\left(\mathbf{S}^{1}\right)$ is not a simple curve, there is a crossing point $z$ of $\bar{\Psi}\left(\mathbf{S}^{1}\right)$ and one can find a simple closed curve $\gamma$ in $\mathcal{U} \cup\{z\}$ surrounding points of $\mathcal{H}_{\infty}$ (since $\bar{\Psi}\left(\mathbf{S}^{1}\right) \subset \partial \mathcal{H}_{\infty}$ ). This contradicts the maximum principle, exactly as in Lemma 1.12, applied to the map $\mathbf{a} \mapsto f_{\mathbf{a}}^{N}(-\mathbf{a})$ for some large $N$.

THEOREM 1. - The boundary of every bounded hyperbolic component is a Jordan curve.

Proof. - The boundary of a hyperbolic component which is contained in $\mathcal{H}$ is a Jordan curve by Theorem 2 above. The other bounded hyperbolic components are connected components of the interior of a copy of $\mathbf{M}$ (by Corollary 1). Thus the boundary of such a component is the image by a homeomorphism of the boundary of a hyperbolic component of the interior of $\mathbf{M}$. Therefore it is a Jordan curve.

The following result is the analogue of Theorem 3 for the captures components. We can go out of a capture component $\mathcal{U}$ (and stay in $\mathcal{C}$ ) only through the landing point of rays of angles $\xi$ such that $(d-1)^{j} \xi=t_{1}$ where $\mathcal{U} \subset \mathcal{W}^{s}\left(t_{1}\right)$.

THEOREM 4. - Let $\mathcal{U}$ be a connected component of $\mathcal{H}_{i} \backslash \mathcal{H}_{0}$ (with $i \in \mathbf{N}$ ). Any parameter $\mathbf{a} \in \partial \mathcal{U}$ is the landing point of a unique ray $\mathcal{R}_{\mathcal{U}}(\xi)$. In the dynamical plane, $R_{\mathbf{a}}^{r(\mathbf{a})}(\xi)$ converges to $f_{\mathbf{a}}(-\mathbf{a})$, which is not on $\partial B_{\mathbf{a}}$ but $f_{\mathbf{a}}^{i+1}(-\mathbf{a}) \in \partial B_{\mathbf{a}}$. No parameter $\mathbf{a} \in \partial \mathcal{U}$ can be of parabolic type. If $\xi \in \mathbf{Q}$ the parameter $\mathbf{a}$ is of Misiurewicz type. Moreover, let $t_{1}$ be such that $\mathcal{U} \subset \mathcal{W}^{s}\left(t_{1}\right)$ (see Proposition 4.15). Then:

- if $(d-1)^{j} \xi=t_{1}$, there are exactly two external parameter rays converging to $\mathbf{a}$;

- otherwise there is exactly one external parameter ray converging to a.

Proof. - The proof is similar to that of Theorem 3. The boundary $\partial \mathcal{U}$ is locally connected by Theorem 2. So, the coordinate $\Phi_{\mathcal{U}}^{-1}: \mathbf{D} \rightarrow \mathcal{U}$ extends to a continuous map from the closure $\overline{\mathbf{D}}$ to $\overline{\mathcal{U}}$. Hence, any point a of $\partial \mathcal{U}$ is the landing point of a unique ray $\mathcal{R}_{\mathcal{U}}(\xi)$. Let $U_{\mathbf{a}}$ be the 


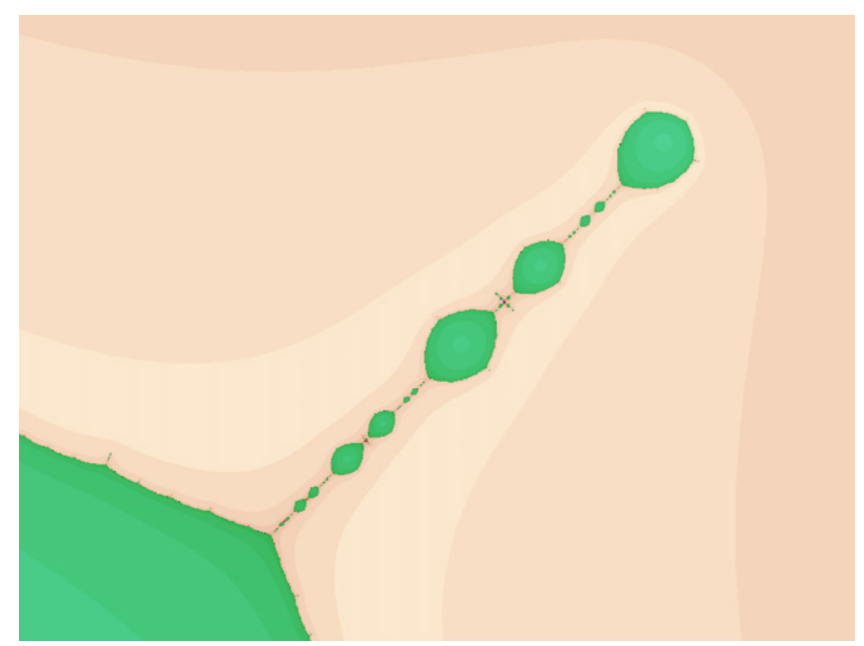

Fig. 12. Zoom on a point of $\partial \mathcal{U}$ where $\mathcal{U} \neq \mathcal{H}_{0}$ is a capture.

connected component of $\widetilde{B}_{\mathbf{a}}$ containing $f_{\mathbf{a}}(-\mathbf{a})$ for $\mathbf{a} \in \mathcal{U}$, let $r(\mathbf{a})$ be its center. The dynamical ray $R_{\mathbf{a}}^{r(\mathbf{a})}(\xi)$ converges to a point $z(\mathbf{a}) \in \partial U_{\mathbf{a}}$ since $\partial U_{\mathbf{a}}$ is locally connected.

If $f_{\mathbf{a}}$ is not renormalizable then $z(\mathbf{a})=f_{\mathbf{a}}(-\mathbf{a})$ :

As in the proof of Theorem 3 , the point $z(\mathbf{a})$ belongs to every $P_{n}^{\mathbf{a}}$ for $n \geqslant 0$ so that $\bigcap P_{n}^{\mathbf{a}}=f_{\mathbf{a}}(-\mathbf{a})$. Thus $z(\mathbf{a})=f_{\mathbf{a}}(-\mathbf{a})$.

If $f_{\mathbf{a}}$ is renormalizable then $z(\mathbf{a})$ is (eventually) parabolic or $z(\mathbf{a})=f_{\mathbf{a}}(-\mathbf{a})$ :

If $f_{\mathbf{a}}$ is renormalizable, Proposition 4.15 insures that $\xi$ is rational (with the uniqueness proved just above). Then $z(\mathbf{a})$ is either a (eventually) parabolic point or $z(\mathbf{a})=f_{\mathbf{a}}(-\mathbf{a})$ (see Lemma 2.24).

The critical value is not on $\partial B_{\mathbf{a}}$ :

Assume, to get a contradiction, that $f_{\mathbf{a}}(-\mathbf{a}) \in \partial B_{\mathbf{a}}$. Then, it is the landing point of exactly one ray of $B_{\mathbf{a}}$, say $R_{\mathbf{a}}^{0}\left(\xi^{\prime}\right)$. Note that the parameter a cannot be of parabolic type since $f_{\mathbf{a}}(-\mathbf{a})$ is not in a Fatou component. Then $z(\mathbf{a})=f_{\mathbf{a}}(-\mathbf{a})$ by the two remarks above. Thus the two rays $f_{\mathbf{a}}^{i}\left(R_{\mathbf{a}}^{r(\mathbf{a})}(\xi)\right)=R_{\mathbf{a}}^{0}(\xi)$ and $f_{\mathbf{a}}^{i}\left(R_{\mathbf{a}}^{0}\left(\xi^{\prime}\right)\right)=R_{\mathbf{a}}^{0}\left((d-1)^{i} \xi^{\prime}\right)$ converge to $f_{\mathbf{a}}^{i}(z(\mathbf{a}))$. By Lemma 1.6, this implies that $(d-1)^{i} \xi^{\prime}=\xi$. Then, the two different rays $R_{\mathbf{a}}^{0}\left((d-1)^{i-1} \xi^{\prime}\right)$ and $f_{\mathbf{a}}^{i-1}\left(R_{\mathbf{a}}^{r(\mathbf{a})}(\xi)\right)$ landing at $f_{\mathbf{a}}^{i-1}(z(\mathbf{a}))$ have the same image by $f_{\mathbf{a}}$. Thus, $f_{\mathbf{a}}^{i-1}(z(\mathbf{a}))=-\mathbf{a}$, so $f_{\mathbf{a}}^{i}(-\mathbf{a})=-\mathbf{a}$ but this is not possible since the critical point would be periodic on the Julia set.

The parameter a is not parabolic:

Assume to get a contradiction that $\mathbf{a}$ is of parabolic type. Then, by Proposition $1, f_{\mathbf{a}}$ is renormalizable, so that $\xi$ is in $\mathbf{Q}$ and $z(\mathbf{a})$ is an (eventually) parabolic periodic point. Proposition 4.15 insures that $\mathcal{U}$ is in some wake $\mathcal{W}\left(\mathbf{a}_{1}\right)$ where $\mathbf{a}_{1} \in \partial \mathcal{H}_{0}$. Then $\mathbf{a} \in \overline{\mathcal{W}\left(\mathbf{a}_{1}\right)}$, but in $\mathcal{W}\left(\mathbf{a}_{1}\right)$ the point $z(\mathbf{a})$ should be eventually repelling, so $\mathbf{a}=\mathbf{a}_{1}$. Hence, the four parameter rays (of Proposition 4.15) $\mathcal{R}_{\infty}\left(\zeta_{1}\right), \mathcal{R}_{\infty}\left(\zeta_{1}^{\prime}\right), \mathcal{R}_{\infty}(\eta)$ and $\mathcal{R}_{\infty}\left(\eta^{\prime}\right)$ land at the same point $\mathbf{a}_{1}$. But this is not possible by Theorem 3 since $\mathbf{a}_{1} \in \partial \mathcal{H}_{0}$.

If $\xi$ is rational $\mathbf{a}$ is of Misiurewicz type:

The conclusion of the points before is that $z(\mathbf{a})=f_{\mathbf{a}}(-\mathbf{a})$. Then $f_{\mathbf{a}}(-\mathbf{a})$ is eventually repelling (since $\xi$ is eventually periodic by multiplication by $d$ ). Thus a is of Misiurewicz type.

The iterate $f_{\mathbf{a}}^{i+1}(-\mathbf{a})$ belongs to $\partial B_{\mathbf{a}}$ :

Indeed, $z(\mathbf{a})=f_{\mathbf{a}}(-\mathbf{a}) \in \partial U_{\mathbf{a}}$ so that $f_{\mathbf{a}}^{i}\left(f_{\mathbf{a}}(-\mathbf{a})\right) \in \partial B_{\mathbf{a}}$.

Finally, we consider the number of external parameter rays converging to $\mathbf{a}$ : 
We have seen above that $\mathbf{a}$ is of Misiurewicz type and that the ray $R_{\mathbf{a}}^{r(\mathbf{a})}(\xi)$ is landing at $f_{\mathbf{a}}(-\mathbf{a})$.

First case: $(d-1)^{j} \xi=t_{1}$. Since $\mathbf{a} \in \mathcal{W}\left(\mathbf{a}_{1}\right)$, the rays $R_{\mathbf{a}}^{0}\left(t_{1}\right), R_{\mathbf{a}}^{\infty}\left(\zeta_{1}\right)$ and $R_{\mathbf{a}}^{\infty}\left(\zeta_{1}^{\prime}\right)$ land at a common point $y$. We can pull back these rays and get two external rays $R_{\mathbf{a}}^{\infty}(\eta)$ and $R_{\mathbf{a}}^{\infty}\left(\eta^{\prime}\right)$ landing at the same point as $R_{\mathbf{a}}^{r(\mathbf{a})}(\xi)$, that is at $f_{\mathbf{a}}(-\mathbf{a})$. Since a is of Misiurewicz type, the external parameter rays $\mathcal{R}_{\infty}(\eta), \mathcal{R}_{\infty}\left(\eta^{\prime}\right)$ land at a. Assume (to get a contradiction) that there is a third external parameter ray $\mathcal{R}_{\infty}\left(\xi^{\prime}\right)$ converging to $\mathbf{a}, \xi^{\prime} \notin\left\{\eta, \eta^{\prime}\right\}$. Then, since a is of Misiurewicz type, the ray $R_{\mathbf{a}}^{\infty}\left(\xi^{\prime}\right)$ lands at $f_{\mathbf{a}}(-\mathbf{a})$ (Lemma 2.24). Thus, since $(d-1)^{j} \xi=t_{1}$, $f_{\mathbf{a}}^{i}\left(R_{\mathbf{a}}^{\infty}\left(\xi^{\prime}\right)\right)$ gives a third external ray converging to $y$, since $d^{i} \xi^{\prime} \notin\left\{\zeta_{1}, \zeta_{1}^{\prime}\right\}$. But this contradicts Lemma 4.10 which insures that at most two external rays land at a point of $\partial B_{\mathbf{a}}$.

Now we do the other cases. Assume (to get a contradiction) that there are (at least) two external dynamical rays converging to $f_{\mathbf{a}}(-\mathbf{a})$. Since $f_{\mathbf{a}}(-\mathbf{a})$ is not on $\partial B_{\mathbf{a}}$ but $y=f_{\mathbf{a}}^{i}\left(f_{\mathbf{a}}(-\mathbf{a})\right) \in \partial B_{\mathbf{a}}$, we iterate the three rays $i$-times and get $R_{\mathbf{a}}^{0}(\xi)$ and two external rays, say $R_{\mathbf{a}}^{\infty}\left(\theta_{1}\right)$ and $R_{\mathbf{a}}^{\infty}\left(\theta_{2}\right)$, converging to $y$. The two external rays separate $\mathbf{C}$ into two connected components. Let $V$ be the one containing $B_{\mathbf{a}}$ and $V_{0}$ be the other one. Note that, as long as the $V_{j}=f^{j}\left(V_{0}\right)$ does not contain a critical point of $f_{\mathbf{a}}$, its image by $f_{\mathbf{a}}$ is still a sector not containing $B_{\mathbf{a}}$ and attached by $f^{i+1}(y)$ to $\partial B_{\mathbf{a}}$ (since $-\mathbf{a}$ is not in the orbit of $y$ ). From Lemma 4.10, some iterate of $V_{0}$ has to contain a critical point. If $V_{j}$ contains 0 , then $V_{j-1}$ contains $B_{\mathrm{a}}^{\prime}$ and so the preimage of $f^{j}(y)$ on $\partial B_{\mathbf{a}}^{\prime}$. Then $f_{\mathbf{a}}: V_{j-1} \rightarrow V_{j}$ is not a homeomorphism so $-\mathbf{a} \in V_{j-1}$. We consider the first $j$ such that $-\mathbf{a} \in V_{j-1}$. The sector $V_{j-1}$ contains a preimage of $\overline{R_{\mathbf{a}}^{\infty}\left(d^{j} \theta_{1}\right)}$ and of $\overline{R_{\mathbf{a}}^{\infty}\left(d^{j} \theta_{2}\right)}$ landing at a preimage of $f_{\mathbf{a}}^{j}(y)$. Let $V_{j}^{\prime}$ be the connected component of $\mathbf{C} \backslash\left(\overline{R_{\mathbf{a}}^{\infty}\left(d^{j} \theta_{1}\right)} \cup \overline{R_{\mathbf{a}}^{\infty}\left(d^{j} \theta_{2}\right)}\right)$ containing the critical value. Denote by $V_{j-1}^{\prime}$ the connected component of $f_{\mathbf{a}}^{-1}\left(V_{j}^{\prime}\right)$ containing -a. Then $V_{j-1}^{\prime} \subset V_{j-1}$. Applying Lemma 4.10 to $V_{j}^{\prime}$, we get that some iterate $f_{\mathbf{a}}^{r}\left(V_{j}^{\prime}\right)$ contains $-\mathbf{a}$, so if $r$ is the smallest iterate to satisfy this condition one has: $f_{\mathbf{a}}^{r}\left(V_{j}^{\prime}\right)=V_{j-1}$. Hence the landing point $f_{\mathbf{a}}^{j-1}(y)=f_{\mathbf{a}}^{j+r}(y)$ is a repelling periodic point. We thicken the sector $V_{j-1}$ by adding a small disk $D$ around $f_{\mathbf{a}}^{j-1}(y)$ which satisfies $f_{\mathbf{a}}^{r+1}(D) \supset \bar{D}$ and by taking external rays close to $R_{\mathbf{a}}^{\infty}\left(d^{j-1} \theta_{1}\right)$ and $R_{\mathbf{a}}^{\infty}\left(d^{j-1} \theta_{2}\right)$ landing at points in $D$. Denote by $V_{j+r}^{\prime \prime}$ this new domain: the domain $V_{j+r}^{\prime \prime}$ is bounded by these two rays union a part of $\partial D$ and containing $D \cup V_{j-1}$. Take the inverse image of $V_{j+r}^{\prime \prime}$ by $f_{\mathbf{a}}^{r+1}$ along the previous orbit (i.e. backward along the orbit $\left.\left\{f_{\mathbf{a}}^{j-1}(y), \ldots, f_{\mathbf{a}}^{j+r}(y)\right\}\right)$. We obtain a domain $V_{j-1}^{\prime \prime}$ with $\overline{V_{j-1}^{\prime \prime}} \subset V_{j+r-1}^{\prime \prime}$ and $f_{\mathbf{a}}^{r}: V_{j-1}^{\prime \prime} \rightarrow V_{j+r-1}^{\prime \prime}$ is proper of degree two. Hence, since $y=f_{\mathbf{a}}^{i}\left(f_{\mathbf{a}}(-\mathbf{a})\right)$, the forward orbit by $f_{\mathbf{a}}^{r+1}$ of $-\mathbf{a}$ will stay in $V_{j-1}^{\prime \prime}$ so that $f_{\mathbf{a}}$ is renormalizable. This contradicts the fact that two external rays converge to $f_{\mathbf{a}}(-\mathbf{a})$ in the non-renormalizable case.

Assume now that two external rays $\mathcal{R}_{\infty}\left(\xi^{\prime}\right), \mathcal{R}_{\infty}\left(\xi^{\prime \prime}\right)$ land at a. These two rays enter any para-puzzle piece $\mathcal{P}_{n}(\mathbf{a})$ so by the homeomorphism of Corollary 3.20 the rays $R_{\infty}^{\mathrm{a}}\left(\xi^{\prime}\right)$ and $R_{\infty}^{\mathbf{a}}\left(\xi^{\prime \prime}\right)$ enter all the pieces $P_{n}^{\mathbf{a}}$. Since the intersection $\bigcap_{n \geqslant 0} P_{n}^{\mathbf{a}}$ reduces to $f_{\mathbf{a}}(-\mathbf{a})$, the rays both converge to the same point $z=f_{\mathbf{a}}(-\mathbf{a})$. But we have just seen that this is not possible.

COROLLARY 3. - There is no parameter a on the boundary of a connected component of $\mathcal{H}$ such that $f_{\mathbf{a}}$ has an irrational indifferent periodic point.

Proof. - Let $\mathcal{U}$ be a connected component of $\mathcal{H}$ and let a be a parameter of $\partial \mathcal{U}$. Assume to get a contradiction that $f_{\mathbf{a}}$ admits an irrational indifferent periodic point denoted by $x$. If $\mathcal{U}=\mathcal{H}_{0}$, Proposition 1 asserts that $f_{\mathbf{a}}$ is renormalizable, then Proposition 4.6 implies that $\mathbf{a}$ is of parabolic type. If $\mathcal{U} \in \mathcal{H}_{i} \backslash \mathcal{H}_{0}$ for some $i>0$, Theorem 4 gives that $f_{\mathbf{a}}^{i+1}(-\mathbf{a}) \in \partial B_{\mathbf{a}}$. Therefore $x \in \partial B_{\mathbf{a}}$ since a subsequence of $\left(f_{\mathbf{a}}^{n}(-\mathbf{a})\right)$ accumulates $x$. Denote by $R_{\mathbf{a}}^{0}(\xi)$ the ray landing at $x\left(\partial B_{\mathbf{a}}\right.$ is a Jordan curve). Since $x$ is periodic, $\xi \in \mathbf{Q}$ (Lemma 1.6). This contradicts the Snail Lemma (see [12]) which asserts that the landing point of a periodic ray either is a repelling periodic point or has multiplier equal to 1 . 


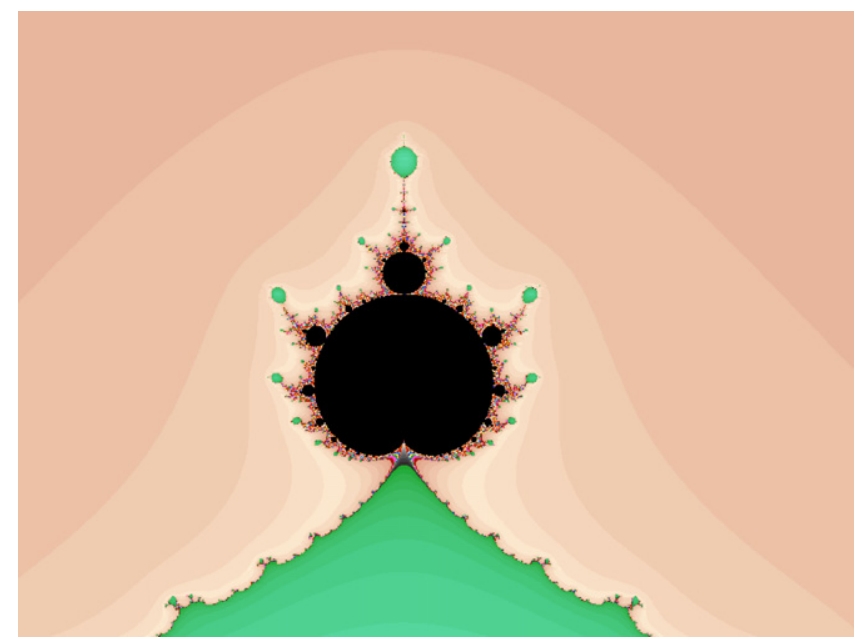

Fig. 13. Components $\mathcal{U}$ attached to the tips of a copy $\mathbf{M}_{0}$ of $\mathbf{M}$.

\section{Description of $\mathcal{C}$ and size of the limbs of $\mathcal{H}_{0}$}

\subsection{Connections in $\mathcal{C}$}

Definition 5.1. - Let $\mathbf{M}_{0}$ be a copy of $\mathbf{M}$, let $\mathcal{U}$ be a hyperbolic component. We say that $\mathbf{M}_{0}$ and $\mathcal{U}$ are attached if $\mathbf{M}_{0}$ intersects $\overline{\mathcal{U}}$.

Definition 5.2. - A tip of $\mathbf{M}$ is the landing point of an external ray of the form $\mathcal{R}_{\mathbf{M}}\left(p / 2^{n}\right)$ with $0<p<2^{n}$. Equivalently it is a parameter $c$ such that $P_{c}^{j}(0)=\beta_{c}$ for some $j>0$ where $\beta_{c}$ is the non-separating fixed point of $K\left(P_{c}\right)$ (here $P_{c}(z)=z^{2}+c$ ).

A tip of a copy $\mathbf{M}_{0}$ of $\mathbf{M}$ is the image $\chi^{-1}(c)$ of a tip $c$ of $\mathbf{M}$, where $\mathbf{M}_{0}=\chi^{-1}(\mathbf{M})$.

Proposition 2. - Let $\mathbf{M}_{0}$ be a copy of $\mathbf{M}$ and $\mathcal{U}$ be any connected component of $\mathcal{H}$. If the intersection $\mathbf{M}_{0} \cap \overline{\mathcal{U}} \neq \emptyset$, then it reduces to exactly one point. Moreover this intersection point is:

- the cusp of $\mathbf{M}_{0}$, if $\mathcal{U}=\mathcal{H}_{0}$

- a tip of $\mathbf{M}_{0}$, if $\mathcal{U} \neq \mathcal{H}_{0}$. Moreover, in this case $\mathbf{M}_{0}$ is also attached to $\mathcal{H}_{0}$ (by its cusp).

Proof. - Let a be in $\mathbf{M}_{0} \bigcap \partial \mathcal{U}$. Since $f_{\mathbf{a}}$ is renormalizable, the intersection $\mathbf{M}_{\mathbf{a}}=\cap \mathcal{P}_{n}(\mathbf{a})$ is a copy of $\mathbf{M}$ (Proposition 4.15). We first prove that $\mathbf{M}_{0}=\mathbf{M}_{\mathbf{a}}$.

If $\mathcal{U}=\mathcal{H}_{0}$, the puzzle pieces $\mathcal{P}_{n}(\mathbf{a})$ intersect $\mathcal{H}_{0}$ along a sector that contains a in its boundary. Thus, they cannot cut $\operatorname{Card}_{0}$, the main cardioid of $\mathbf{M}_{0}$. Then $\operatorname{Card}_{0}$ is contained in every $\mathcal{P}_{n}(\mathbf{a})$ so in the intersection $\mathbf{M}_{\mathbf{a}}$. Thus $\mathbf{M}_{\mathbf{a}}=\mathbf{M}_{0}$. The proof of Theorem 3 insures that $\mathbf{a}$ is the cusp of $\mathbf{M}_{\mathbf{a}}$. Hence, $\mathbf{M}_{0} \cap \partial \mathcal{H}_{0}=\{\mathbf{a}\}$ the cusp of $\mathbf{M}_{0}$.

If $\mathcal{U} \neq \mathcal{H}_{0}, \mathbf{M}_{\mathbf{a}}$ is attached to $\mathcal{H}_{0}$ by Proposition 4.15 . Hence $\mathbf{M}_{\mathbf{a}}=\mathbf{M}_{0}$, otherwise one can find a loop in $\mathbf{M}_{\mathbf{a}} \cup \mathbf{M}_{0} \cup \overline{\mathcal{H}}_{0}$ surrounding points of $\mathcal{H}_{\infty}$, which contradicts the fact that $\mathcal{H}_{\infty} \cup\{\infty\}$ is connected. Then, from Proposition 4.15 we get that $\mathbf{M}_{\mathbf{a}} \cap \mathcal{U}=\{\mathbf{a}\}$. Now we prove that $\mathbf{a}$ is a tip of $\mathbf{M}_{0}$. From Theorem 4 , the parameter $\mathbf{a}$ is of Misiurewicz type since $x:=f_{\mathbf{a}}^{i+1}(-\mathbf{a}) \in \partial B_{\mathbf{a}}$ is an (eventually) repelling periodic point. Some iterate $z=f_{\mathbf{a}}^{r}(x)$ belongs to $K\left(f_{\mathbf{a}}^{k}\right)=\cap P_{n}^{\mathbf{a}}$. Then $z$ is a repelling fixed point of $f_{\mathbf{a}}^{k}$ which does not separate $K\left(f_{\mathbf{a}}^{k}\right)$. So $z$ corresponds to the $\beta$ fixed point of $z^{2}+\chi(\mathbf{a})$, the quadratic polynomial to which $f_{\mathbf{a}}^{k}$ is conjugated. Therefore, $\mathbf{a}$ is a tip of $\mathbf{M}_{0}$.

$4^{\text {e }}$ SÉRIE - TOME $40-2007-\mathrm{N}^{\circ} 6$ 
COROLlaRY 5.3. - Let $\mathbf{M}_{0}$ be a copy of $\mathbf{M}$ attached to $\mathcal{H}_{0}$ and contained in a wake $\mathcal{W}^{s}(t)$. Let $\mathcal{U}$ be a connected component of $\mathcal{H} \backslash \mathcal{H}_{0}$ attached to $\mathbf{M}_{0}$. The landing point of an internal ray of $\mathcal{U}, \mathcal{R}_{\mathcal{U}}(\xi)$, is a tip of $\mathbf{M}_{0}$ if and only if $\xi=t$.

Proof. - Let a be the landing point of $\mathcal{R}_{\mathcal{U}}(\xi)$ and let $\mathbf{a}_{0}$ be the intersection point $\overline{\mathcal{U}} \cap \mathbf{M}_{0}$ (there is only one point by Proposition 2). We prove first that $\mathbf{a}_{0}$ is the landing point of $\mathcal{R}_{\mathcal{U}}(t)$.

Since $f_{\mathbf{a}_{0}}$ is renormalizable we can apply Proposition 4.15. Namely, the intersection $\mathbf{M}_{\mathbf{a}_{0}}=$ $\cap \mathcal{P}_{n}\left(\mathbf{a}_{0}\right)$ is attached to $\mathcal{H}_{0}$ and the intersection point is the landing point of $\mathcal{R}_{0}(t)$, since $\mathbf{a}_{0} \in \mathcal{W}^{s}(t)$ and since the curve $\overline{\mathcal{R}_{\infty}(\zeta)} \cup \overline{\mathcal{R}_{\infty}\left(\zeta^{\prime}\right)}$ separates $\overline{\mathcal{H}}_{0}$ from the points of $\mathcal{W}^{s}(t)$. Thus, $\mathbf{M}_{0}=\mathbf{M}_{\mathbf{a}_{0}}$; otherwise one can find a loop in $\mathbf{M}_{0} \cup \mathbf{M}_{\mathbf{a}_{0}} \cup \overline{\mathcal{H}}_{0}$ surrounding points of $\mathcal{H}_{\infty}$ which contradicts the fact that $\mathcal{H}_{\infty} \cup\{\infty\}$ is connected. Then Proposition 4.15 insures that $\mathbf{a}_{0}$ is the landing point of $\mathcal{R}_{\mathcal{U}}(t)$.

Now, if $\mathbf{a}$ is a tip of $\mathbf{M}_{0}, \mathbf{a}=\mathbf{a}_{0}$ since it is the only intersection point between $\overline{\mathcal{U}}$ and $\mathbf{M}_{\mathbf{a}_{0}}$ (Proposition 2). Therefore $\mathbf{a}$ is the landing point of $\mathcal{R}_{\mathcal{U}}(t)$ and $t=\xi$ by the uniqueness of Theorem 4.

Conversely, if $\xi=t$ since $\mathbf{a}$ is the landing point of $\mathcal{R}_{\mathcal{U}}(\xi)$ and $\mathbf{a}_{0}$ is the landing point of $\mathcal{R}_{\mathcal{U}}(t)$, $\mathbf{a}=\mathbf{a}_{0}$ so that $\mathbf{a}$ is a tip of $\mathbf{M}_{0}$ (Proposition 2).

Proposition 3. - If a copy $\mathbf{M}_{0}$ of $\mathbf{M}$ is attached to $\mathcal{H}_{0}$, then at any of its tips, there is a connected component $\mathcal{U}$ of $\mathcal{H} \backslash \mathcal{H}_{0}$ which is attached.

Proof. - Let a be a tip of $\mathbf{M}_{0}$ and $\mathbf{a}_{0}=\mathbf{M}_{0} \cap \overline{\mathcal{H}}_{0} ; \mathbf{a}_{0}$ is the cusp of $\mathbf{M}_{0}$ by Proposition 2. Moreover, $\mathbf{M}_{0}=\mathbf{M}_{\mathbf{a}_{0}}$ and $\mathcal{P}_{n}(\mathbf{a})=\mathcal{P}_{n}\left(\mathbf{a}_{0}\right)$ for all $n \geqslant 0$, also from the proof of Proposition 2 above. Since $\mathbf{M}_{0}$ is attached to $\mathcal{H}_{0}$ every para-puzzle piece $\mathcal{P}_{n}(\mathbf{a})$ intersects $\mathcal{H}_{0}$. Therefore, the puzzle pieces $P_{n}^{\mathbf{a}}$ intersect the basin $B_{\mathbf{a}}$ (applying the homeomorphism of Corollary 3.20). Then, the intersection $K_{\mathbf{a}}=\cap P_{n}^{\mathbf{a}}$ intersects $\bar{B}_{\mathbf{a}}$ and since $K\left(f_{\mathbf{a}}\right)$ is full, $K_{\mathbf{a}} \cap \bar{B}_{\mathbf{a}}$ reduces to one point, say $x$. Since $f_{\mathbf{a}}$ is renormalizable, there exists $k>0$ such that $f_{\mathbf{a}}^{k}$ maps $P_{n+k}^{\mathbf{a}}$ onto $P_{n}^{\mathbf{a}}$, so the point $x$ is $k$-periodic. Since $x \in \partial B_{\mathbf{a}}, x$ is the landing point of a unique ray, say $R_{\mathbf{a}}^{0}(\tau)$. The uniqueness implies that $\tau$ is $k$-periodic by multiplication by $d-1$, so that $x$ is the non-separating fixed point of the renormalized map $f_{\mathbf{a}}^{k}$. Since $\mathbf{a}$ is a tip of $\mathbf{M}_{0}, f_{\mathbf{a}}(-\mathbf{a})$ is mapped by some iterate of $f_{\mathbf{a}}^{k}$ to $x$. Thus some iterated preimage $R_{\mathbf{a}}^{r(\mathbf{a})}(\tau)$ of $R_{\mathbf{a}}^{0}(\tau)$ is landing at $f_{\mathbf{a}}(-\mathbf{a}) \neq x$ with $r(\mathbf{a})$ the center of some connected component $U_{\mathbf{a}}$ of $\widetilde{B}_{\mathbf{a}} \backslash B_{\mathbf{a}}$ (since $K\left(f_{\mathbf{a}}\right)$ is full). The puzzle pieces $P_{n}^{\mathbf{a}}$ intersect $U_{\mathbf{a}}$ for all $n \geqslant 0$. Denote by $R_{\mathbf{a}}^{r(\mathbf{a})}\left(\tau_{n}\right)$ and $R_{\mathbf{a}}^{r(\mathbf{a})}\left(\tau_{n}^{\prime}\right)$ the rays involved in $U_{\mathbf{a}} \cap \partial P_{n}^{\mathbf{a}}$ and by $R_{\mathbf{a}}^{\infty}\left(\eta_{n}\right)$, respectively $R_{\mathbf{a}}^{\infty}\left(\eta_{n}^{\prime}\right)$, the external rays of $\partial P_{n}^{\mathbf{a}}$ converging to the landing points of the rays in $U_{\mathbf{a}}$. By the homeomorphism of Corollary 3.20, the para-puzzle pieces $\mathcal{P}_{n}(\mathbf{a})$ should intersect some component $\mathcal{U}$ of $\mathcal{H} \backslash \mathcal{H}_{0}$ and the rays involved in $\partial \mathcal{P}_{n}(\mathbf{a}) \cap \mathcal{U}$ are $R_{\mathcal{U}}\left(\tau_{n}\right)$ and $R_{\mathcal{U}}\left(\tau_{n}^{\prime}\right)$ converging to the same points as the external rays $R_{\infty}\left(\eta_{n}\right)$ and $R_{\infty}\left(\eta_{n}^{\prime}\right)$ respectively (at least for infinitely many $n \in \mathbf{N}$ ). The sequences $\left(\tau_{n}\right),\left(\tau_{n}^{\prime}\right)$ converge to $\tau$ and the sequence $\left(\eta_{n}\right)$, resp. $\left(\eta_{n}^{\prime}\right)$, converges to $\eta$, resp. $\eta^{\prime}$ by Propositions 4.6 and 4.15 . In the dynamical plane $R_{\mathbf{a}}^{r(\mathbf{a})}(\tau), R_{\mathbf{a}}^{\infty}(\eta)$ and $R_{\mathbf{a}}^{\infty}\left(\eta^{\prime}\right)$ converge to the same point $f_{\mathbf{a}}(-\mathbf{a})$. Since a is a Misiurewicz parameter, $\mathcal{R}_{\infty}(\eta)$ lands at a (Lemma 2.26). Assume that the internal ray $\mathcal{R}_{\mathcal{U}}(\tau)$ lands at a parameter $\mathbf{a}^{\prime} \neq \mathbf{a}$. Since $\mathcal{R}_{\mathcal{U}}(\tau)$ enters every puzzle pieces $\mathcal{P}_{n}(\mathbf{a})$, the parameter $\mathbf{a}^{\prime}$ belongs to $\mathbf{M}_{\mathbf{a}}=\mathbf{M}_{0}$. This contradicts the fact that $\mathcal{R}_{\infty}(\eta)$ will have to land at $\mathbf{a}^{\prime}$ by Proposition 4.15. So $\mathcal{U}$ is attached to $\mathbf{M}_{0}$ at $\mathbf{a}$.

LEMMA 1. - Any two distinct components of $\mathcal{H}$ have disjoint closures.

Proof. - Assume, to get a contradiction, that there exist $\mathcal{U}_{1}, \mathcal{U}_{2}$ two distinct components of $\mathcal{H}$ and $\mathbf{a} \in \overline{\mathcal{U}}_{1} \cap \overline{\mathcal{U}}_{2}$. Since one connected component is distinct from $\mathcal{H}_{0}$, the parameter a is a Misiurewicz point by Theorem 4. Moreover Theorem 4 and Theorem 3 insure that there exist $\xi_{1}$, $\xi_{2}$ such that $\mathcal{R}_{\mathcal{U}_{1}}\left(\xi_{1}\right), \mathcal{R}_{\mathcal{U}_{2}}\left(\xi_{2}\right)$ land at $\mathbf{a}$ and $R_{\mathbf{a}}^{r_{1}(\mathbf{a})}\left(\xi_{1}\right), R_{\mathbf{a}}^{r_{2}(\mathbf{a})}\left(\xi_{2}\right)$ land at $f_{\mathbf{a}}(-\mathbf{a})$ for some 


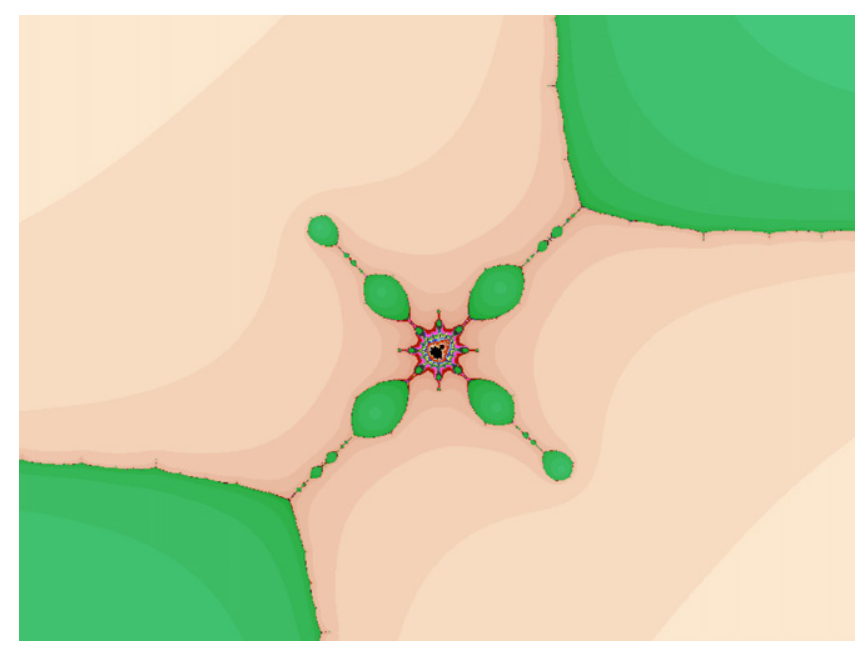

Fig. 14. At the center a Mandelbrot copy without connections with $\mathcal{H}$.

adapted centers $r_{1}(\mathbf{a}), r_{2}(\mathbf{a})$. This is impossible since $f_{\mathbf{a}}(-\mathbf{a})$ would be eventually critical by Lemma 2.21, although the critical point cannot be periodic on the Julia set.

THEOREM 5. - The only intersections between closures of hyperbolic components, and also copies of $\mathbf{M}$ are the following:

- the central component $\mathcal{H}_{0}$ has Mandelbrot copies $\mathbf{M}_{t}$ attached to it at angles $t$ which are $(d-1)$-periodic (a full characterization of these values is given in Proposition 2.32):

- at every tip of such a satellite $\mathbf{M}_{t}$, a capture component $\mathcal{U}$ of $\mathcal{H} \backslash \mathcal{H}_{0}$ is attached.

Nevertheless, there are infinitely many copies of $\mathbf{M}$ in $\mathcal{C}$ and infinitely many captures components not contained in the category described above.

Proof. - The description of the intersections of copies of $\mathbf{M}$ with components of $\mathcal{H}$ follows from Proposition 2 and 3. The intersections between components of $\mathcal{H}$ follow from Lemma 1.

There are other copies of $\mathbf{M}$ in $\mathcal{C}$ (see Fig. 14) which are not attached to components of $\mathcal{H}$ since Mandelbrot copies are dense in $\mathcal{C}$ by [11].

We prove now that there are capture components $\mathcal{V}$ of $\mathcal{H} \backslash \mathcal{H}_{0}$ which are not attached to Mandelbrot copies (and also not attached to hyperbolic components by Lemma 1).

We start with a capture component $\mathcal{U}$ attached to some satellite copy $\mathbf{M}_{t}$. Let a be the landing point of $\mathcal{R}_{\mathcal{U}}(\xi)$ with $(d-1) \xi=t$. In the dynamical plane for a, the critical value is on the boundary of some component $U_{\mathbf{a}}$ which is mapped by some $f_{\mathbf{a}}^{i}$ to $B_{\mathbf{a}}$. Since $\mathcal{U}$ is in $\mathcal{W}^{s}(t)$, the critical value $f_{\mathbf{a}}(-\mathbf{a})$ is separated from $B_{\mathbf{a}}$ by two external rays $R_{\mathbf{a}}^{\infty}\left(\theta_{1}\right), R_{\mathbf{a}}^{\infty}\left(\theta_{2}\right)$ landing at $f_{\mathbf{a}}^{i}\left(f_{\mathbf{a}}(-\mathbf{a})\right)$ a repelling periodic point (Proposition 4.15 and Lemma 4.14). Then, considering the puzzle pieces, there is some $n$ such that $\partial P_{n}^{\mathbf{a}}$ involves rays in $U_{\mathbf{a}}$ and other rays in some iterated inverse image of $U_{\mathbf{a}}$ denoted by $V_{\mathbf{a}}$. Using the homeomorphism of Corollary 3.20 we get that $\partial \mathcal{P}_{n}(\mathbf{a}) \cap \mathcal{P}_{n-1}(\mathbf{a})$ contains rays in some capture component $\mathcal{V}$ (because of the external parameter rays). This para-puzzle piece is contained in $\mathcal{W}^{s}(t)$ so that $\mathcal{V}$ is contained in $\mathcal{W}^{s}(t)$. Therefore, $\mathcal{V}$ cannot be attached to a Mandelbrot copy. Otherwise this copy is attached to $\mathcal{H}_{0}$ (Proposition 4.15) so would coincide with $\mathbf{M}_{0}$. Therefore $\mathcal{U}$ and $\mathcal{V}$ cannot be connected by an arc avoiding $\mathbf{M}_{0}$, else one can complete it by an arc in $\mathcal{U} \cup \mathbf{M}_{0} \cup \mathcal{V}$ to get a loop in $\mathcal{C}$ surrounding points of $\mathcal{H}_{\infty}$ which contradicts the fact that $\mathcal{H}_{\infty} \cup\{\infty\}$ is connected. But since $\mathcal{C} \cap \mathcal{P}_{n}(\mathbf{a})$

$4^{\mathrm{e}}$ SÉRIE - TOME $40-2007-\mathrm{N}^{\circ} 6$ 
is connected, we get that $\mathcal{U}$ and $\mathcal{V}$ are connected outside $\mathbf{M}_{0}$. Hence, $\mathcal{V}$ is not attached to a Mandelbrot copy.

\subsection{Size of the limbs}

DEFINITION 5.4. - For any $\mathbf{a} \in \partial \mathcal{H}_{0}$, we denote by limb containing a the intersection $\mathcal{L}(\mathbf{a})=$ $\overline{\mathcal{W}(\mathbf{a})} \cap \mathcal{C}$ if $\mathcal{W}(\mathbf{a}) \neq \emptyset$ and else $\mathcal{L}(\mathbf{a})=\{\mathbf{a}\}$

Remark 5.5. - By Lemma 4.14, the definition of the limbs coincides with the one given by Milnor in [14].

Remark 5.6. - Note that $\mathcal{L}(\mathbf{a}) \cap \partial \mathcal{H}_{0}=\{\mathbf{a}\}$. We will adopt sometimes the notation $\mathcal{L}^{s}(t)$ for a $\operatorname{limb} \mathcal{L}(\mathbf{a})$ where $\mathbf{a}$ is the landing point of $\mathcal{R}_{0}^{s}(t)$.

The following result was conjectured in [14] (with another parametrization of the rays). It follows from Theorem 3 .

COROLlaRY 5.7. $-A$ limb $\mathcal{L}^{s}(t)$ contains more than one point if and only if the angle $\frac{t}{d-1}+\frac{\left\lfloor\frac{d-1}{2}\right\rfloor}{d-1}$ is periodic by multiplication by $d-1$.

Proof. - Let $\mathbf{a}_{0}$ be the point of $\partial \mathcal{H}_{0} \cap \mathcal{L}^{s}(t)$, so $\mathcal{L}^{s}(t)=\mathcal{L}\left(\mathbf{a}_{0}\right)$. From Theorem 3 there are two external rays converging to $\mathbf{a}_{0}$ if and only if $\mathbf{a}_{0}$ is of parabolic type. This corresponds to the statement about periodicity of Proposition 2.32.

THEOREM 7. - For any $\epsilon>0$, there exists only a finite number of limbs with diameter greater than $\epsilon$.

Proof. - This proof is inspired by Milnor's one in the quadratic case. It proceeds in two steps.

1. Every point of $\mathcal{C} \backslash \mathcal{H}_{0}$ belongs to a unique limb.

Let $\mathbf{a} \in \mathcal{C} \cap \mathcal{S} \backslash \mathcal{H}_{0}$. For every $n$, there exists a sector $S_{n}(\mathbf{a})$ containing a such that $\mathcal{X}_{n} \cap \partial S_{n}(\mathbf{a}) \subset \mathcal{I}_{n}$ is the union of two rays in $\mathcal{H}_{0}$ and two external rays. This sector contains a para-puzzle piece, say $\mathcal{P}_{n}$, which intersects $\mathcal{H}_{0}$ along two internal rays $\mathcal{R}_{0}\left(t_{n}\right), \mathcal{R}_{0}\left(t_{n}^{\prime}\right)$ and $\mathcal{H}_{\infty}$ along two external rays $\mathcal{R}_{\infty}\left(\zeta_{n}\right), \mathcal{R}_{\infty}\left(\zeta_{n}^{\prime}\right)$.

The study of the para-puzzle pieces (Lemma 4.2, Proposition 4.6) gives the following informations. The sequences of angles $t_{n}, t_{n}^{\prime}$ converge to the common limit $t$. Let $\mathbf{a}_{0}$ be the landing point of $\mathcal{R}_{0}(t)$. Assume that $f_{\mathbf{a}_{0}}$ is renormalizable. Then the sequences $\left(\zeta_{n}\right)$, $\left(\zeta_{n}^{\prime}\right)$ converge to different angles: $\zeta$ and $\zeta^{\prime}$ respectively, with $\mathcal{R}_{\infty}(\zeta), \mathcal{R}_{\infty}\left(\zeta^{\prime}\right)$ landing at $\mathbf{a}_{0}$ (Theorem 3). Thus a belongs to the wake $\mathcal{W}\left(\mathbf{a}_{0}\right)$ so to the limb $\mathcal{L}(t)$. If, $f_{\mathbf{a}_{0}}$ is not renormalizable, $\mathbf{a}=\mathbf{a}_{0}$ since $\mathbf{a} \in \cap \mathcal{P}_{n}=\left\{\mathbf{a}_{0}\right\}$, so $\cap P_{n}^{\mathbf{a}_{0}}=f_{\mathbf{a}}(-\mathbf{a})$ and $\cap \mathcal{P}_{n}=\{\mathbf{a}\}$. If two external parameter rays enter the para-puzzle pieces $\mathcal{P}_{n}$, by the homeomorphism of Corollary 3.20 the two corresponding dynamical rays enter the puzzle pieces $P_{n}^{\mathbf{a}_{0}}$, so converge to $f_{\mathbf{a}_{0}}\left(-\mathbf{a}_{0}\right)$. This contradicts Theorem 3 . Therefore the two external rays $\mathcal{R}_{\infty}\left(\zeta_{n}\right)$ and $\mathcal{R}_{\infty}\left(\zeta_{n}^{\prime}\right)$ converge to an external ray $\mathcal{R}_{\infty}(\zeta)$ which lands at $\mathbf{a}_{0}$. Thus $\mathcal{W}\left(\mathbf{a}_{0}\right)=\emptyset$ and $\mathbf{a}=\mathbf{a}_{0}=\mathcal{L}(t)$.

2. We assume (to get a contradiction) that there exists a sequence $\mathcal{L}\left(t_{n}\right)$ of limbs which accumulates at two points $\mathbf{a}_{1} \neq \mathbf{a}_{2}$.

First suppose that $\mathbf{a}_{1}, \mathbf{a}_{2}$ belong to two different limbs $\mathcal{L}\left(t_{1}\right)$ and $\mathcal{L}\left(t_{2}\right)$ respectively. There exists $t \in\left(t_{1}, t_{2}\right)$ such that $\mathcal{R}_{0}(t)$ lands at a Misiurewicz parameter $\mathbf{a}$. Then this point is the landing point of an external ray $\mathcal{R}_{\infty}(\zeta)$. The curve $\mathcal{R}_{0}(t) \cup \mathcal{R}_{\infty}(\zeta)$ separates $\mathbf{a}_{1}$ from $\mathbf{a}_{2}$ so that the sequence $\mathcal{L}\left(t_{n}\right)$ cannot accumulate on both $\mathbf{a}_{1}$ and $\mathbf{a}_{2}$.

Now suppose that $\mathbf{a}_{1}$ and $\mathbf{a}_{2}$ belong to the same limb $\mathcal{L}(t)$, we can assume that $\mathbf{a}_{1} \notin \partial \mathcal{H}_{0}$ (since $\partial \mathcal{H}_{0}$ is locally connected), so there exists $r>0$ such that $B\left(\mathbf{a}_{1}, r\right) \subset \mathcal{W}\left(\mathbf{a}_{1}\right)$. Therefore, 
if a sequence $\mathbf{a}_{n} \in \mathcal{L}\left(t_{n}\right)$ accumulates $\mathbf{a}_{1}$, then for large $n$ the parameter $\mathbf{a}_{n}$ belongs to the ball $B(\mathbf{a}, r)$ so to $\mathcal{W}\left(\mathbf{a}_{1}\right)$ and therefore to $\mathcal{L}(t)$ so $t_{n}=t$.

\subsection{Local connectivity of $\partial \mathcal{C}$}

THEOREM 6. $-\partial \mathcal{C}$ is locally connected at every point which is not in a copy of $\mathbf{M}$ and at any point of $\partial \mathcal{U}$ for every connected component $\mathcal{U}$ of $\mathcal{H}$.

Proof. - Corollary 4 gives the local connectivity for parameters which are not in a copy of $\mathbf{M}$. Let $\mathbf{a}_{0} \in \partial \mathcal{U}$ where $\mathcal{U}$ is a connected component of $\mathcal{H}_{i}$ and $\mathcal{P}_{n}$ be the para-puzzle piece containing $\mathbf{a}_{0}$. If $\mathbf{a}_{0}$ is not parabolic, $\cap \mathcal{P}_{n}=\left\{\mathbf{a}_{0}\right\}$ then $\partial \mathcal{C}$ is locally connected at this point by Lemma 4.4. If $\mathbf{a}_{0}$ is parabolic, then the intersection $\cap \mathcal{P}_{n}$ is a copy $\mathbf{M}_{0}$ of $\mathbf{M}$. So to get the local connectivity at $\mathbf{a}_{0}$ we consider the restricted puzzle pieces $\mathcal{P}_{n}^{\prime}$ as follows. Let $\gamma$ be the parametrization by the internal angle of the boundary of the main cardioid of $\mathbf{M}_{0}$ with $\gamma(0)$ at the cusp. We consider an external ray converging to $\gamma(1 / n)$ and another to $\gamma(-1 / n)$ then we complete their union to get with $\infty$ a closed curve $\delta_{n}$ by adding some segment of curve $c_{n}$ inside the main cardioid. Let $\mathcal{P}_{n}^{\prime}$ be the connected component of $\mathcal{P}_{n} \backslash \delta_{n}$ containing $\mathbf{a}_{0}$. The choice of $c_{n}$ is such that the intersection of $\mathcal{P}_{n}^{\prime}$ with the cardioid of $\mathbf{M}_{0}$ is a sequence decreasing to the cusp of $\mathbf{M}_{0}$ (the boundary of cardioid is locally connected). We prove now that the sequence $\mathcal{P}_{n}^{\prime}$ is a basis of neighbourhoods of $\mathbf{a}_{0}$. Assume that there is a point $\mathbf{a} \neq \mathbf{a}_{0}$ in $\cap \mathcal{P}_{n}^{\prime}$. The parameter $\mathbf{a}$ is not on the cardioid of $\mathbf{M}_{0}$, otherwise it would be some $\gamma(t)$ with $\frac{-1}{n} \leqslant t \leqslant \frac{1}{n}$ so it can only be $\mathbf{a}_{0}$. Then, since the intersection of $\mathcal{P}_{n}$ is $\mathbf{M}_{0}$, the parameter a belongs to a Limb of $\mathbf{M}_{0}$ (the image of a limb of $\mathbf{M}$ by the homeomorphism) say $\mathcal{L}_{\mathbf{M}}(t)$. Thus for $n$ such that $1 / n<|t|$ the point a does not belong to $\mathcal{P}_{n}^{\prime}$. This gives the contradiction. The intersection $\mathcal{C} \cap \mathcal{P}_{n}^{\prime}$ is clearly connected by the same arguments as in Lemma 4.4 since we cut nicely the piece $\mathcal{P}_{n}$ with the curve $\delta_{n}$.

\section{Acknowledgements}

I would like to thank Tan Lei, Carsten Petersen and Curt Mc Mullen for encouraging me to write this down. I would also like to thank John Milnor for many discussions and for suggesting me Section 2.5.

\section{Appendix}

For the completeness of the article, we recall here the proof of the following result:

THEOREM [7,17]. - The boundary of every connected component of $\widetilde{B}_{\mathrm{a}}$ is a Jordan curve.

\section{A.1. Yoccoz' Theorem for rational-like maps}

Definition A.1. - A map $f: X^{\prime} \rightarrow X$ is rational-like if:

- $X, X^{\prime}$ are connected open sets of $\overline{\mathbf{C}}$ with smooth boundary, such that $X \supset \overline{X^{\prime}}$ and $\partial X$ has a finite number of connected components;

- $f: X^{\prime} \rightarrow X$ is a holomorphic proper map with a finite number of critical points and extends to a continuous map from $\overline{X^{\prime}}$ to $\bar{X}$.

For a rational-like map $f: X^{\prime} \rightarrow X$, a graph $\Gamma$ is admissible if:

$-\Gamma$ is connected, finite, included in $\bar{X}$ and contains $\partial X$;

- $\Gamma$ is stable, i.e. $f^{-1}(\Gamma) \supset \Gamma \cap X^{\prime}$;

- the forward orbits of the critical points are disjoint from $\Gamma$.

$4^{\mathrm{e}}$ SÉRIE - TOME $40-2007-\mathrm{N}^{\circ} 6$ 
To an admissible graph, $\Gamma$, a puzzle is associated:

DEFINITION A.2. - The puzzle pieces of depth $n$ are by definition the connected components of $f^{-n}(X \backslash \Gamma)$.

The end of a point $x$ is the nested sequence $\left(P_{0}(x), P_{1}(x), \ldots, P_{n}(x), \ldots\right)$ of the puzzle pieces containing $x$.

The end of $x$ is periodic if there exist $k, N$ such that $f^{k}\left(P_{k+n}(x)\right)=P_{n}(x)$ for $n \geqslant N$.

The impression of $x$ is the intersection $\bigcap_{n \geqslant 0} P_{n}(x)$ of the puzzle pieces containing $x$.

The point $x$ is surrounded at depth $i$ if the annulus $A_{i}=P_{i} \backslash \bar{P}_{i+1}$ surrounds $x$, i.e. if $x \in P_{i+1}$ and $\overline{P_{i+1}} \subset P_{i}$.

Yoccoz' Theorem can be stated in the context of rational-like maps as follows:

THEOREM. - Let $f: X^{\prime} \rightarrow X$ be a rational-like map with a unique critical point $x_{0}$ of multiplicity 2 and $x$ be a point of $K(f)$. If $\Gamma$ is an admissible graph that surrounds $x_{0}$ and surrounds infinitely many times $x$ then:

- if the end of $x_{0}$ is not periodic, then the impression of $x$ is equal to $\{x\}$;

- otherwise, let $k$ be the period of the end of $x_{0}$, the map $f^{k}: P_{l+k}\left(x_{0}\right) \rightarrow P_{l}\left(x_{0}\right)$ is quadraticlike for l large enough and the impression of $x_{0}$ is the filled Julia set $K\left(\left.f^{k}\right|_{P_{l+k}\left(x_{0}\right)}\right)$ of the renormalized map. Moreover the impression of $x$ reduces to $x$ or to a preimage of the impression of $x_{0}$ if some iterate of $x$ falls in the impression of $x_{0}$.

Remark A.3. - Let $C$ be a forward invariant set under a rational-like map $f$. A compactness argument shows that instead of finding one admissible graph and infinitely many annuli surrounding $x \in C$ with this graph, it is enough to find a finite number of admissible graphs $\Gamma_{0}, \ldots, \Gamma_{l}$ such that every point of $C$ is surrounded at bounded depth by one of these graphs which surrounds also the critical point $x_{0}$.

\section{A.2. Application to the family $f_{\text {a }}$}

It is enough to prove that $B_{\mathbf{a}}$ is locally connected: one gets then the result for every connected component of $\widetilde{B}_{\mathbf{a}}$ by pulling back.

Remark A.4. - If $\mathbf{a} \notin \mathcal{C}$ the connected components of the Julia set are locally connected since $f_{\mathbf{a}}$ is hyperbolic. Thus we consider only parameters $\mathbf{a} \in \mathcal{C}$.

Let $X$ be the connected component of $\overline{\mathbf{C}} \backslash\left(E_{\mathbf{a}}^{\infty}(1) \cup E_{\mathbf{a}}^{0}(1)\right)$ containing $J\left(f_{\mathbf{a}}\right)$ and $X^{\prime}=$ $f_{\mathbf{a}}^{-1}(X)$. The map $f_{\mathbf{a}}: X^{\prime} \rightarrow X$ is a rational-like map. We consider the graphs given in Section 3. They are clearly admissible. We prove now that they satisfy the conditions of Yoccoz' theorem: using Remark A.3 it suffices to show that every point $x \in \partial B_{\mathbf{a}}$ is surrounded, at bounded depth, by one of the graphs which also surrounds the critical point $-\mathbf{a}$.

LEMMA A.5. - For $\theta=\frac{1}{(d-1)^{l}-1}$ and $\theta^{\prime}=\frac{1}{(d-1)^{l^{\prime}}-1}$ with $l^{\prime}>l+1$ and l large enough, every point of $\partial B_{\mathrm{a}}$ is surrounded by one of the graphs at bounded depth.

Proof. - Let $U(\theta)$ be the connected component of $\overline{\mathbf{C}} \backslash \gamma$ containing $R_{\mathbf{a}}^{0}(0)$ where $\gamma$ denotes the curve in $I_{1}^{\mathbf{a}}(\theta)=f_{\mathbf{a}}^{-1}\left(I_{0}^{\mathbf{a}}(\theta)\right)$ formed by the internal rays $R_{\mathbf{a}}^{0}\left(\theta+\frac{1}{d-1}\right)$ and $R_{\mathbf{a}}^{0}\left(\frac{\theta}{d-1}\right)$ and the corresponding external rays. One sees that every point of $\partial B_{\mathbf{a}}$ which is in $U(\theta)$ but not on the graph $I_{1}^{\mathbf{a}}(\theta)$ is surrounded at depth 0 by the graph $I_{0}(\theta)$. Now the points on $\partial B_{\mathbf{a}}$ of $I_{1}(\theta)$ and of $I_{1}\left(\theta^{\prime}\right)$ are distinct. Moreover the union $V=U(\theta) \cup U\left(\theta^{\prime}\right)$ is the connected component of the complement of $\delta$ containing $R_{\mathbf{a}}^{0}(0)$, where $\delta$ is the curve formed by the internal rays $R_{\mathbf{a}}^{0}\left(\theta^{\prime}+\frac{1}{d-1}\right)$ and $R_{\mathbf{a}}^{0}\left(\frac{\theta}{d-1}\right)$ and the corresponding external rays. Then for $n$ such 
that $\frac{1}{n}\left(\theta^{\prime}+\frac{1}{d-1}\right)<\frac{\theta}{d-1}$, the union $\bigcup_{i \leqslant n} f_{\mathbf{a}}^{-i}(V)$ covers $\mathbf{C} \backslash\{0\}$. Thus every point of $\partial B_{\mathbf{a}}$ is surrounded at depth less than $n$ by $I_{0}(\theta)$ or by $I_{0}\left(\theta^{\prime}\right)$.

Remark A.6. - This result clearly holds also for $\theta=\frac{-1}{(d-1)^{l}-1}$ and $\theta^{\prime}=\frac{-1}{(d-1)^{l^{\prime}}-1}$.

LemmA A.7. - There exist $k_{0}$ and $\epsilon \in\{ \pm 1\}$ (depending on a) such that for every $k>k_{0}$ the critical point $-\mathbf{a}$ is surrounded at depth 1 by the graph $I_{0}(\epsilon \theta)$ where $\theta=\frac{1}{(d-1)^{k}-1}$.

Proof. - We take the open set $U(\theta)$ defined in previous lemma. For $d>3$ it is clear that the union $U(\theta) \cup U(-\theta)$ covers $B_{\mathbf{a}} \backslash\{0\}$ and therefore all $\mathbf{C} \backslash\{0\}$. This solves the question for $d>3$.

For $d=3$, the union $U(\theta) \cup U(-\theta) \cup f_{\mathbf{a}}^{-1}(U(\theta) \cup U(-\theta))$ covers all $B_{\mathbf{a}} \backslash\{0\}$ and therefore all $\mathbf{C} \backslash\{0\}$.

The proof that the intersection $\overline{P_{n}(x)} \cap \partial B_{\mathbf{a}}$ is a connected set is exactly the same as the proof of Lemma 4.3 (in the parameter plane).

Yoccoz' Theorem (stated in Appendix A.1) and the previous lemmas allow us to conclude that if the end of the critical point $-\mathbf{a}$ is not periodic, the boundary $\partial B_{\mathbf{a}}$ is locally connected. Then Caratheodory's Theorem together with Lemma 1.6 insures that $\partial B_{\mathrm{a}}$ is a Jordan curve.

We now consider the case where the end of the critical point $-\mathbf{a}$ is periodic of period $k$.

The map $f_{\mathbf{a}}^{k}: P_{n+k}(-\mathbf{a}) \rightarrow P_{n}(-\mathbf{a})$ is quadratic-like and the orbit of the critical point never escapes the puzzle piece $P_{n}(-\mathbf{a})$. So by the straightening theorem of [6], the restriction of $f_{\mathbf{a}}^{k}$ is conjugated to a unique quadratic polynomial $z^{2}+c$. Let $K_{\mathbf{a}}=K\left(f_{\mathbf{a}}^{k}\right)$ denote the filled Julia set of the restriction and $K$ the filled Julia set of the quadratic polynomial.

We assume that $K_{\mathbf{a}} \cap \partial B_{\mathbf{a}} \neq \emptyset$.

LEMMA A.8. - There exists an internal ray $R_{\mathbf{a}}^{0}(\eta)$ of period $k$ converging to the nonseparating fixed point $\beta_{\mathbf{a}}$ of $f_{\mathbf{a}}^{k}$ in $K_{\mathbf{a}}$ and two external rays $R_{\mathbf{a}}^{\infty}(\zeta), R_{\mathbf{a}}^{\infty}\left(\zeta^{\prime}\right)$ converging to $\beta_{\mathbf{a}}$ and separating $K_{\mathbf{a}}$ from $B_{\mathbf{a}}$.

Proof. - The angles of the internal rays that bound the puzzle piece $P_{n}(-\mathbf{a})$ are of the form $\eta_{n}<\eta_{n}^{\prime}$ with $(d-1)^{k} \eta_{n+k}=\eta_{n} \bmod 1$ (and the same for $\eta_{n}^{\prime}$ ) with $\left|\eta_{n}^{\prime}-\eta_{n}\right|<\frac{1}{(d-1)^{n-1}}$. Therefore they converge to a common limit $\eta$ which is periodic of period $k$. Moreover the ray $R_{\mathbf{a}}^{0}(\eta)$ lies in all the puzzles pieces $P_{n}(-\mathbf{a})$ so its landing point is in $\partial B_{\mathbf{a}} \cap K_{\mathbf{a}}$. Since it is a fixed point of $f_{\mathbf{a}}^{k}$ with rotation number 0 it is the non-separating fixed point of $K_{\mathbf{a}}$, i.e. $\beta_{\mathbf{a}}$.

For the external rays the proof is the same. The external rays attached to $R_{\mathbf{a}}^{0}\left(\eta_{n}\right)$ and $R_{\mathbf{a}}^{0}\left(\eta_{n}^{\prime}\right)$ which are in the boundary of $P_{n}(-\mathbf{a})$ are of the form $R_{\mathbf{a}}^{0}\left(\zeta_{n}\right)$ and $R_{\mathbf{a}}^{0}\left(\zeta_{n}^{\prime}\right)$ and the angle satisfies the equations $d^{k} \zeta_{n+k}=\zeta_{n}$ and $d^{k} \zeta_{n+k}^{\prime}=\zeta_{n}^{\prime}$ respectively. Thus they converge to periodic angles $\zeta, \zeta^{\prime}$. The rays $R_{\mathbf{a}}^{\infty}(\zeta)$ and $R_{\mathbf{a}}\left(\zeta^{\prime}\right)$ converge to $\beta_{\mathbf{a}}$ by the same argument as before. To see that the curve $R_{\mathbf{a}}^{\infty}(\zeta) \cup R_{\mathbf{a}}^{\infty}\left(\zeta^{\prime}\right) \cup \beta_{\mathbf{a}}$ separates $B_{\mathbf{a}}$ from $K_{\mathbf{a}} \backslash\left\{\beta_{\mathbf{a}}\right\}$ it is enough to note that the preimage $\beta_{\mathbf{a}}^{\prime}$ of $\beta_{\mathbf{a}}$ in $K_{\mathbf{a}}$ is the landing point of a ray of the form $R_{\mathbf{a}}^{\infty}\left(\frac{\zeta}{d}+\frac{i}{d}\right)$ which is always contained in $P_{n}(-\mathbf{a})$ so converges to $K_{\mathbf{a}}$ and separates $R_{\mathbf{a}}^{\infty}(\zeta)$ from $R_{\mathbf{a}}^{\infty}\left(\zeta^{\prime}\right)$.

COROLLARY A.9. - The boundary of $\partial B_{\mathrm{a}}$ is locally connected.

Proof. - When the end of the critical point $-\mathbf{a}$ is periodic, the impression of the end is $K_{\mathbf{a}}$. If $K_{\mathbf{a}} \cap \partial B_{\mathbf{a}}$ is empty the end of any point forms a basis of connected neighbourhoods of that point. If $K_{\mathbf{a}} \cap \partial B_{\mathbf{a}}$ is not empty, we take for sequence of neighbourhoods of $\beta_{\mathbf{a}}$ in $\partial B_{\mathbf{a}}$ the intersection $U_{n}=\overline{V_{n}} \cap \partial B_{\mathbf{a}}$ where $V_{n}$ is the connected component of $P_{n}\left(\beta_{\mathbf{a}}\right) \backslash\left(R_{\mathbf{a}}^{\infty}(\zeta) \cup R_{\mathbf{a}}^{\infty}\left(\zeta^{\prime}\right) \cup \beta_{\mathbf{a}}\right)$ which intersects $B_{\mathbf{a}}$. It is easy to see that the sequence $\left(U_{n}\right)$ forms a basis of connected neighbourhoods of $\beta_{\mathbf{a}}$ in $\partial B_{\mathbf{a}}$ since the intersection $\cap U_{n}$ reduces to $\partial B_{\mathbf{a}} \cap K_{\mathbf{a}}=\beta_{\mathbf{a}}$. Then we pull back those neighbourhoods along the backward orbit of $\beta_{\mathbf{a}}$.

$4^{\text {e }}$ SÉRIE - TOME $40-2007-\mathrm{N}^{\circ} 6$ 


\section{REFERENCES}

[1] Alhfors L.V., Lectures on Quasi-Conformal Mappings, Wadsworth \& Brook/Cole, Advanced Books \& Software, Monterey, 1987.

[2] Blanchard P., Complex analytic dynamics on the Riemann sphere, Bull. Amer. Math. Soc. 11 (1984) 85-141.

[3] Branner B., Puzzles and para-puzzles of quadratic and cubic polynomials, Proc. Symp. Appl. Math. 49 (1994) 31-69.

[4] Branner B., Hubbard J.H., The iteration of cubic polynomials, Part. 1: The global topology of the parameter space, Acta Math. 160 (1988) 143-206.

[5] Doundy A., Hubbard J.H., Étude dynamique des polynômes complexes I \& II, Publ. Math. d'Orsay (1984) \& (1985).

[6] Douady A., Hubbard J.H., On the dynamics of polynomial-like mappings, Ann. Sci. Éc. Norm. Sup. 18 (1985) 287-343.

[7] FAUGHT D., Local connectivity in a family of cubic polynomials, Ph.D. Thesis, Cornell University, 1992.

[8] Goldberg L.R., Milnor J., Fixed points of polynomial maps. Part II. Fixed point portraits, Ann. Sci. Éc. Norm. Sup. 26 (1993) 51-98.

[9] HubBard J.H., Local connectivity of Julia sets and bifurcation loci: three theorems of J.-C. Yoccoz, in: Goldberg L.R., Phillips V.A. (Eds.), Topological Methods in Modern Mathematics, Publish or Perish, 1993, pp. 467-511.

[10] LeVin G., PrZYTYCKi F., External rays to periodic points, Israel J. Math. 94 (1996) 29-57.

[11] Mc Mullen C., The Mandelbrot set is universal, in: Tan Lei (Ed.), The Mandelbrot Set, Theme and Variations, in: LMS Lecture Note Series, vol. 274, Cambridge U. Press, 2000.

[12] Milnor J., Dynamics in One Complex Variable, Vieweg, 1999, 2nd ed. 2000.

[13] Milnor J., Local connectivity of Julia sets: Expository lectures, in: Tan Lei (Ed.), The Mandelbrot Set, Theme and Variations, in: LMS Lecture Note Series, vol. 274, Cambridge U. Press, 2000, pp. 67-116.

[14] Milnor J., On cubic polynomial maps with periodic critical point, preprint (1991), to be published dedicated to JHH.

[15] NAǏSHUL' V.A., Topological invariants of analytic and area-preserving mappings and their application to analytic differential equations in $C^{2}$ and $C P^{2}$, Trans. Moscow Math. Soc. 42 (1983) 239-250.

[16] Petersen C.L., On the Pommerenke-Levin-Yoccoz inequality, Ergod. Th. \& Dynam. Sys. 13 (1993) 785-806.

[17] Roesch P., Puzzles de Yoccoz pour les applications à allure rationnelle, L'Enseignement Mathématique 45 (1999) 133-168.

[18] RoEsCH P., Holomorphic motions and puzzles (following M. Shishikura), in: Tan Lei (Ed.), The Mandelbrot Set, Theme and Variations, in: LMS Lecture Note Series, vol. 274, Cambridge U. Press, 2000, pp. 117-132.

[19] SŁodKowski Z., Extensions of holomorphic motions, Ann. Scuola Norm. Sup. Cl. Sci. 22 (1995) $185-210$.

[20] Yoccoz J.-C., Petits diviseurs en dimension 1, Astérisque 231 (1995).

(Manuscrit reçu le 25 janvier 2007; accepté, après révision, le 4 octobre 2007.)

Pascale RoEsch

IMT, Laboratoire Emile Picard,

Université Paul Sabatier

118 , route de Narbonne,

31062 Toulouse Cedex 9, France

E-mail: pascale.roesch@math.ups-tlse.fr 